\title{
COMPUTER-AIDED DIAGNOSES (CAD) SYSTEM: AN ARTIFICIAL \\ NEURAL NETWORK APPROACH TO MRI ANALYSIS AND \\ DIAGNOSIS OF ALZHEIMER'S DISEASE (AD)
}

\author{
A Thesis \\ presented to \\ the Faculty of California Polytechnic State University, \\ San Luis Obispo
}

\author{
In Partial Fulfillment \\ of the Requirements for the Degree \\ Master of Science in Biomedical Engineering
}

by

Berizohar Padilla Cerezo

August 2017 
(C) 2017

Berizohar Padilla Cerezo

ALL RIGHTS RESERVED 
TITLE:

AUTHOR:

DATE SUBMITTED:

COMMITTEE CHAIR:

COMMITTEE MEMBER:

COMMITTEE MEMBER:
Computer-Aided Diagnoses (CAD) System: An Artificial Neural Network Approach to MRI Analysis and Diagnosis of Alzheimer's Disease (AD)

Berizohar Padilla Cerezo

August 2017

Dr. Lanny Griffin, Ph.D.

Associated Professor of Biomedical Engineering

Dr. Xiao-Hua (Helen) Yu, Ph.D.

Associated Professor of Electrical Engineering

Dr. Michael Witt, Ph.D.

Associated Professor of Biomedical Engineering 


\section{ABSTRACT \\ Computer-Aided Diagnoses (CAD) System: An Artificial Neural Network Approach to MRI Analysis and Diagnosis of Alzheimer's Disease (AD) \\ Berizohar Padilla Cerezo}

Alzheimer's disease (AD) is a chronic and progressive, irreversible syndrome that deteriorates the cognitive functions. Official death certificates of 2013 reported 84,767 deaths from Alzheimer's disease, making it the 6th leading cause of death in the United States. The rate of AD is estimated to double by 2050. The neurodegeneration of AD occurs decades before symptoms of dementia are evident. Therefore, having an efficient methodology for the early and proper diagnosis can lead to more effective treatments.

Neuroimaging techniques such as magnetic resonance imaging (MRI) can detect changes in the brain of living subjects. Moreover, medical imaging techniques are the best diagnostic tools to determine brain atrophies; however, a significant limitation is the level of training, methodology, and experience of the diagnostician. Thus, Computer aided diagnosis (CAD) systems are part of a promising tool to help improve the diagnostic outcomes. No publications addressing the use of Feedforward Artificial Neural Networks (ANN), and MRI image attributes for the classification of AD were found.

Consequently, the focus of this study is to investigate if the use of MRI images, specifically texture and frequency attributes along with a feedforward ANN model, can lead to the classification of individuals with AD. Moreover, this study compared the use of a single view versus a multi-view of MRI images and their performance. The frequency, texture, and MRI views in combination with the feedforward artificial neural network were tested to determine if they were comparable to the clinician's performance. The clinician's performances used were 78 percent accuracy, 87 percent sensitivity, 71 percent specificity, and 78 percent precision from a study with 1,073 individuals.

The study found that the use of the Discrete Wavelet Transform (DWT) and Fourier Transform (FT) low frequency give comparable results to the clinicians; however, the FT outperformed the clinicians with an accuracy of 85 percent, precision of 87 percent, sensitivity of 90 percent and specificity of 75 percent. In the case of texture, a single texture feature, and the combination of two or more features gave results comparable to the clinicians. However, the Gray level co-occurrence matrix (GLCOM), which is the combination of texture features, was the highest performing texture method with 82 percent accuracy, 86 percent sensitivity, 76 percent specificity, and 86 percent precision. Combination CII (energy and entropy) outperformed all other combinations with 78 percent accuracy, 88 percent sensitivity, 72 percent specificity, and 78 percent precision. Additionally, a combination of views can increase performance for certain texture attributes; however, the axial view outperformed the sagittal and coronal views in the case of frequency attributes. In conclusion, this study found that both texture and frequency characteristics in combinations with a feedforward backpropagation neural network can perform at the level of the clinician and even higher depending on the attribute and the view or combination of views used.

Keywords: Alzheimer's disease, artificial neural networks, feedforward, MRI, image attributes, texture, frequency, Fourier Transform, Discrete Wavelet Transform, GLCOM, energy, entropy, correlation, contrast, axial, sagittal, coronal, ADNI 


\section{ACKNOWLEDGMENTS}

This project was inspired by Abraham Milchiker and his fierce fight against Alzheimer's disease. Jack Brittle and the others near me who suffer from mental illnesses. I am thankful for the opportunity to use engineering as a tool to find more efficient methods to approach mental issues.

My greatest thanks are to my advisor Dr. Lenny Griffin for the trust and guidance to complete my masters and to Dr. Helen Yu for her inspiration, patience, guidance, and constant push throughout the completion of this work. I am deeply grateful to Dr. Michael Whitt who has always being a big supporter, mentor, and who has taken the time not only to be part of my committee, but to advise me in my career goals.

I will also like to acknowledge Dr. Kristen O'Halloran Cardinal for her always inspiring, encouraging teachings, attitude, and help with my defense presentation and to Dr. Erika Rogers, who coached me in the completion of my work.

I am eternally grateful to my mom for her love and encouragement, to Cathie Shaffer for her unconditional support, to Crystal Castillo and Sara Della Ripa for her friendship and inspiration, and to all the Cal Poly faculty who have helped me accomplish what I set out to do.

Special thanks to Steven Milchiker for his patience, and provision throughout my education and hardships.

My deepest thanks to Zophia Padilla (my daughter), who patiently spent countless hours at coffee shops working with me, and encouraging me as I completed my work. Also, to Gamaliel who inspires me every day, and to Andre, Ezra, and Zechariah who I hope to inspire one day.

Heartfelt thanks to my sister for her unconditional support, encouragement, and guidance throughout my education and life. I could not have completed this journey without her.

"Let the future tell the truth, and evaluate each one according to his work and accomplishments. The present is theirs; the future, for which I have really worked, is mine."

-Nikola Tesla 


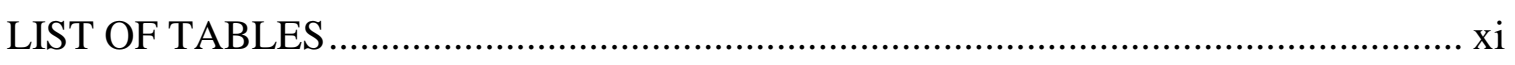

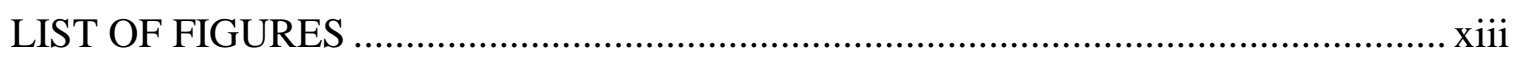

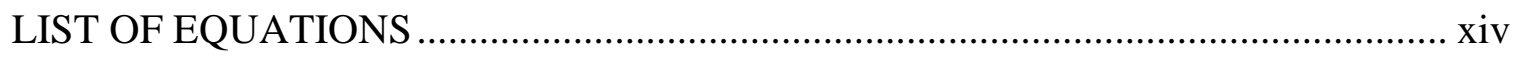

CHAPTER

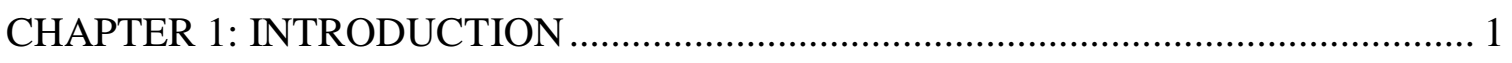

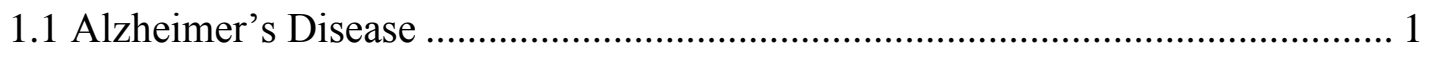

1.1.1 Overview of Alzheimer's disease ...................................................... 1

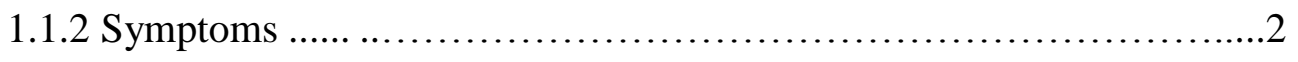

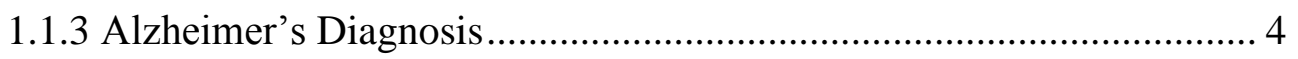

1.1.4 Computer Aided Diagnostic (CAD) system ...................................... 4

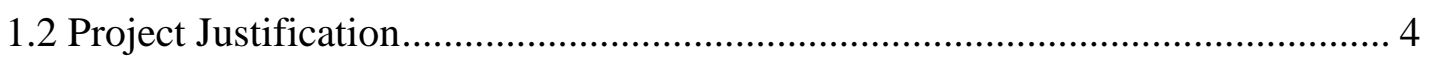

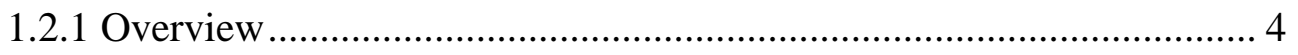

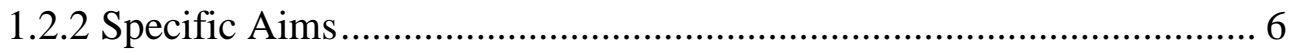

1.2.3 Project Goal ................................................................................... 7

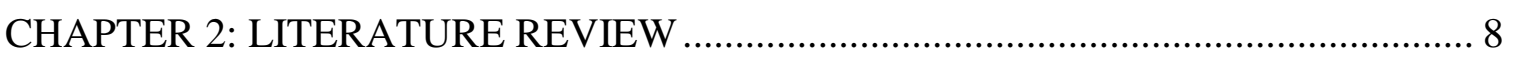

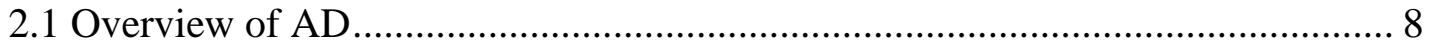

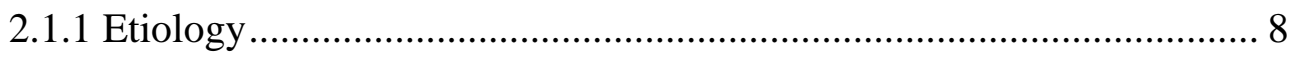

2.1.2 Prevalence and Risk Factors .......................................................... 8

2.1.2.1 Genetic Factors .................................................................... 9

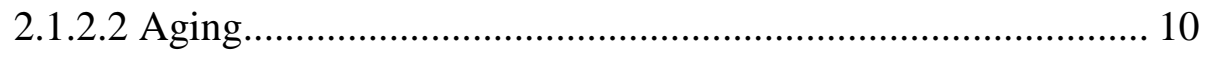

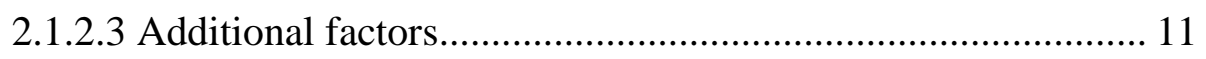

2.1.3 Diagnosis Methods............................................................................ 11

2.1.3.1 Background ............................................................................ 11

2.1.3.2 Recent Diagnosis Modifications ............................................. 12

2.1.4 Imaging as Diagnostic Tools ............................................................ 13

2.1.5 MRI Imaging and Its Limitations ....................................................... 13

2.1.6 Current Treatments ......................................................................... 14

2.2 Computer Aided Diagnosis (CAD) Systems ....................................................... 15

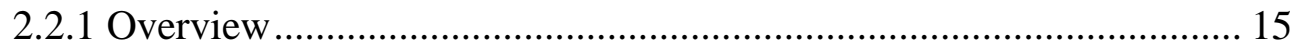




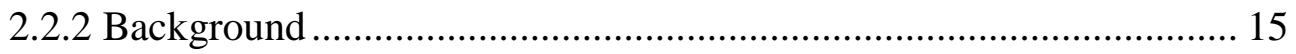

2.2.3 Automated Computer Diagnosis and CAD ......................................... 16

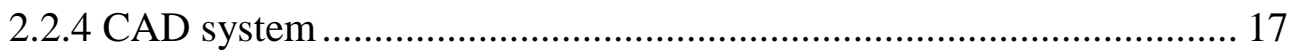

2.2.5 CAD systems performance ............................................................... 17

2.2.6 Artificial Neural Network ................................................................ 18

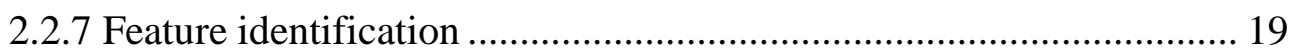

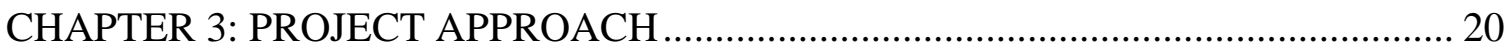

3.1 Background ............................................................................................ 20

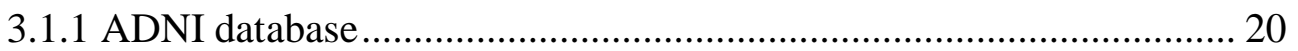

3.1.2 MATLAB neural network toolbox ................................................. 20

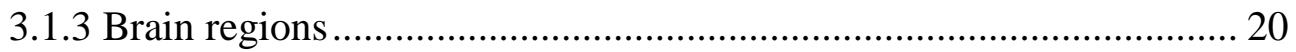

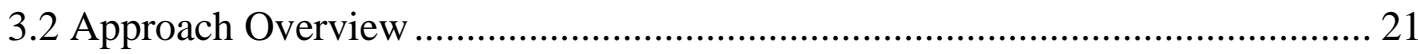

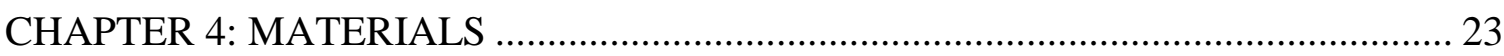

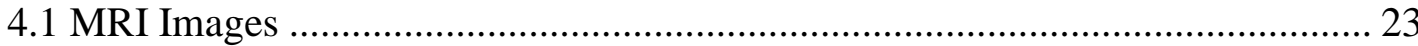

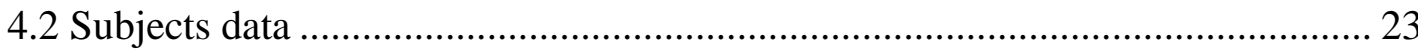

4.2.1 Normal control (NC) subjects and Alzheimer's disease

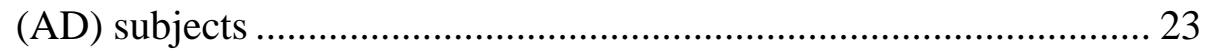

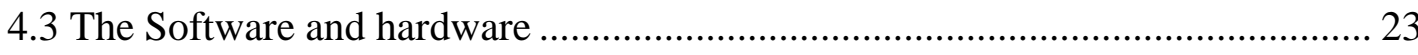

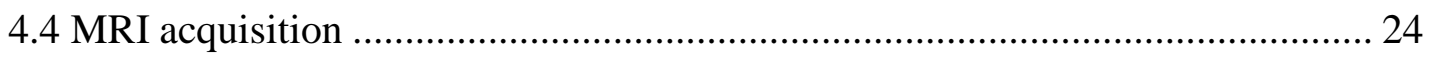

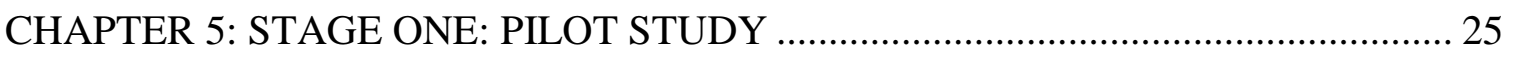

5.1 Materials for pilot study .............................................................................. 25

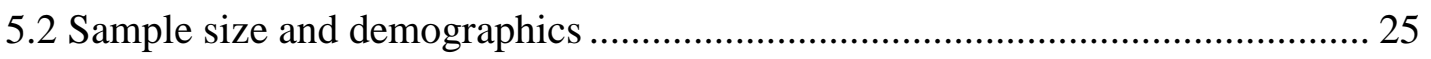

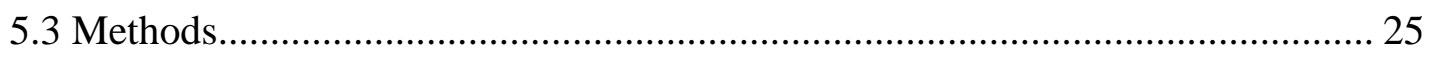

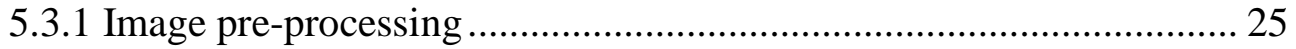

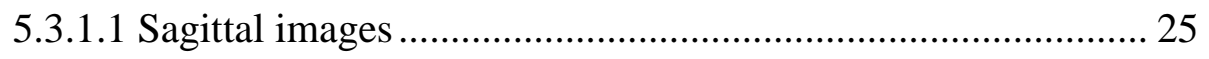

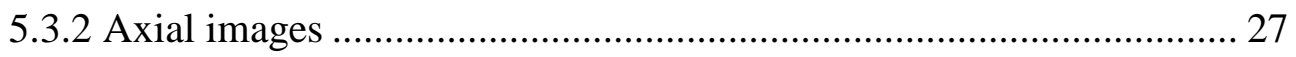

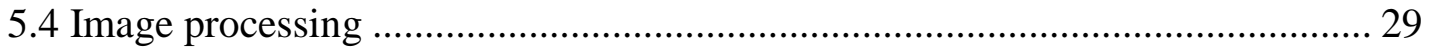

5.4.1 Frequency Domain Processing .............................................................. 30

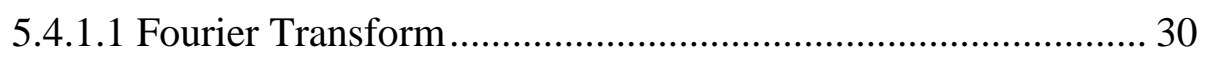

5.4.2 Statistical approach ............................................................................. 31

5.4.2.1 Properties of gray-level co-occurrence matrix.......................... 31 


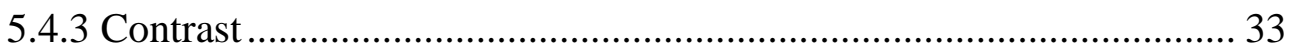

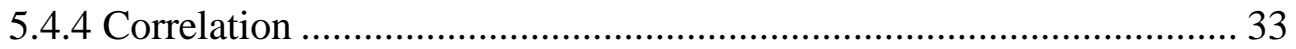

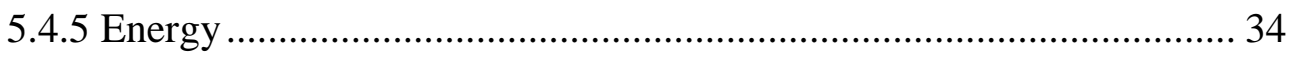

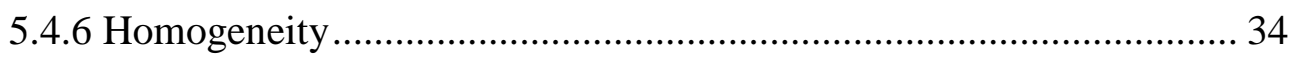

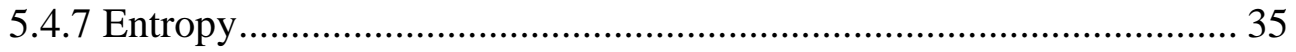

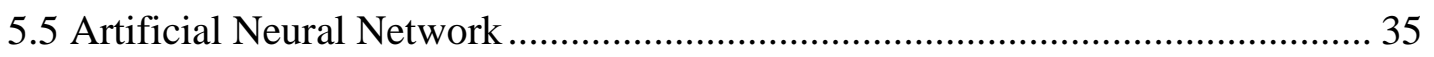

5.5.1 Neural Network structure............................................................... 35

5.5.2 Training Function and Cut off value.................................................. 36

5.6 Stage One Results ...................................................................................... 37

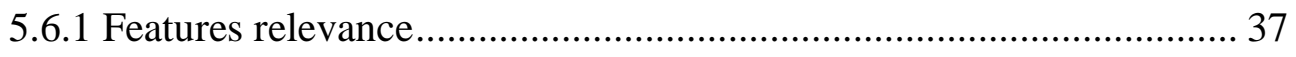

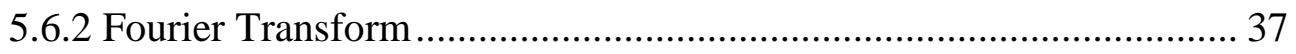

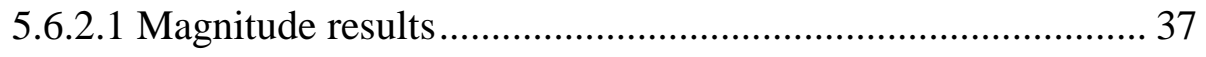

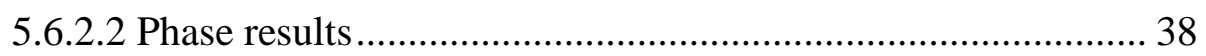

5.6.3 Contrast results.................................................................................. 39

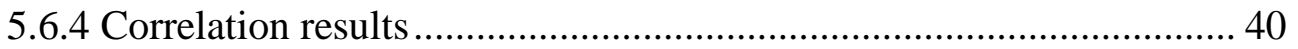

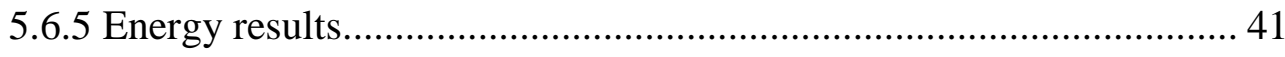

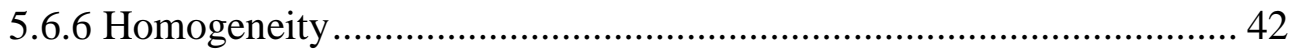

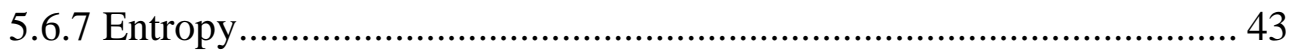

5.7 Artificial Neural Network .......................................................................... 43

5.7.1 Classifier and performance evaluation................................................ 43

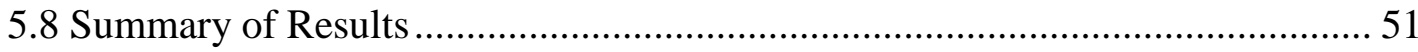

CHAPTER 6: STAGE TWO TEXTURE AND FREQUENCY RESULTS .................... 52

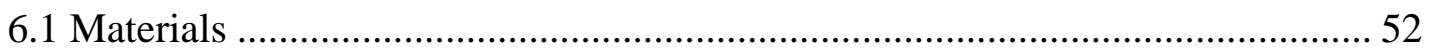

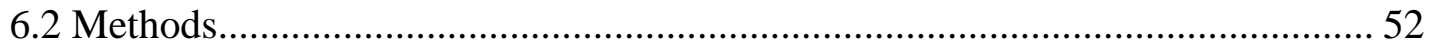

6.3 Transforms comparison at low frequencies ................................................... 53

6.3.1 Fourier Transform (FT) ................................................................ 53

6.3.2 Discrete Wavelet Transform (DWT) ............................................... 53

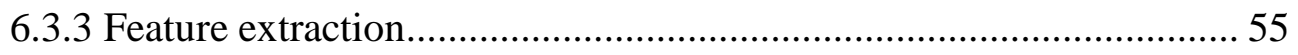

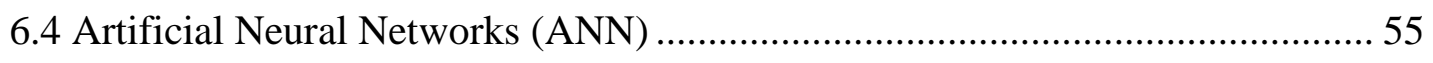

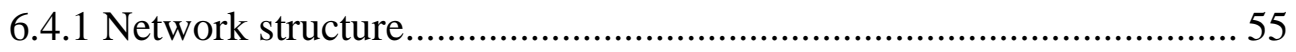

6.4.2 Classifier and performance evaluation................................................. 55 


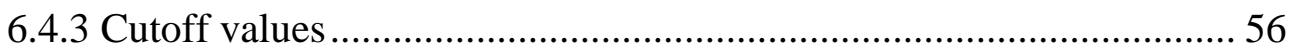

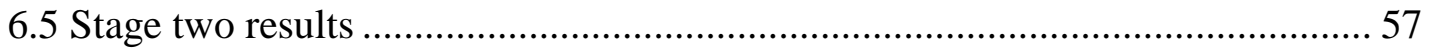

6.5.1 Transform results and comparison................................................. 57

6.5.1.1 Discrete Wavelet Transform (DWT) Results ........................ 57

6.5.1.2 Fourier Transform (FT) Results............................................. 59

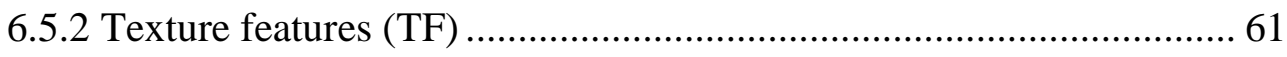

6.5.2.1 Texture features and entropy results ......................................... 61

6.5.2.2 Individual texture features performance ................................. 62

6.5.2.3 Combination of features......................................................... 64

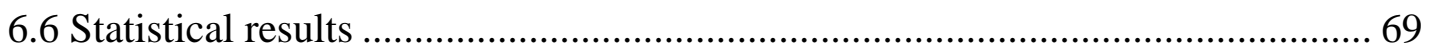

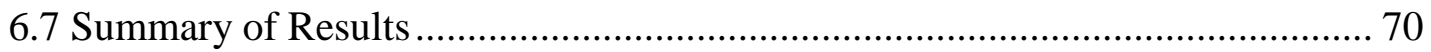

CHAPTER 7: STAGE THREE VIEW COMPARISON RESULTS ............................. 73

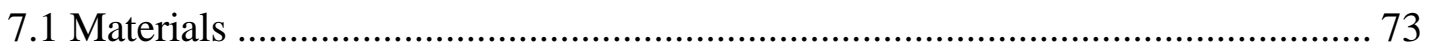

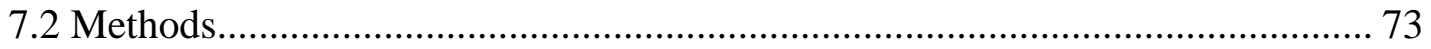

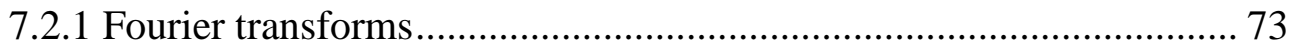

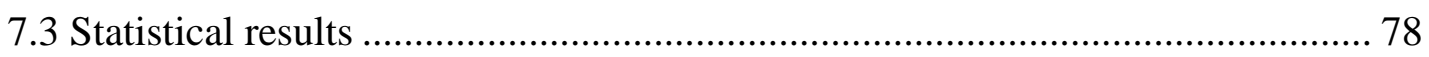

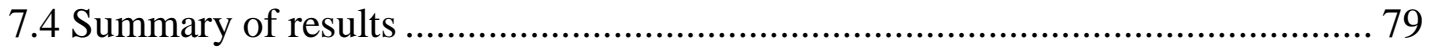

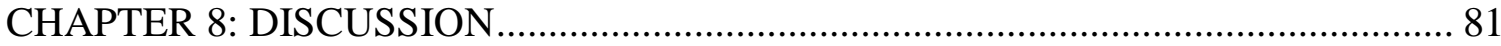

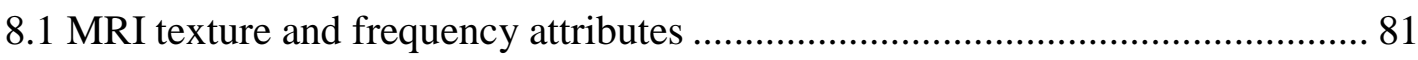

8.2 Single view performance vs. combination of views ........................................ 86

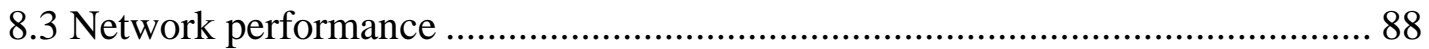

CHAPTER 9: CONCLUSION AND FUTURE WORK .............................................. 91

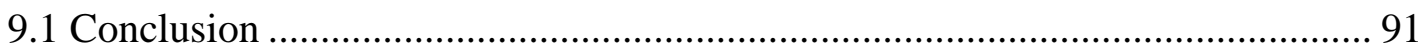

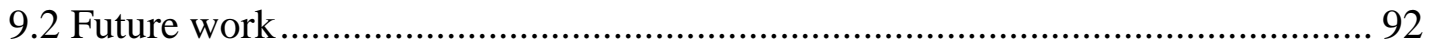

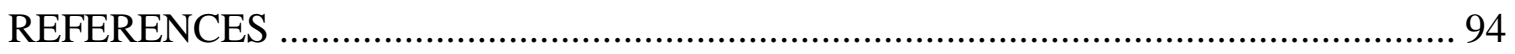

\section{APPENDICES}

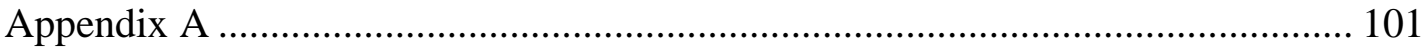

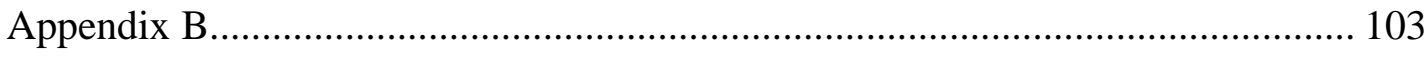

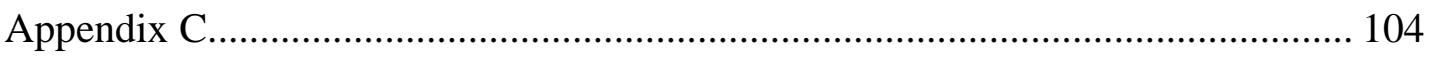

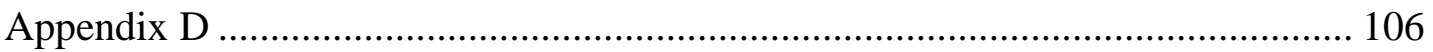

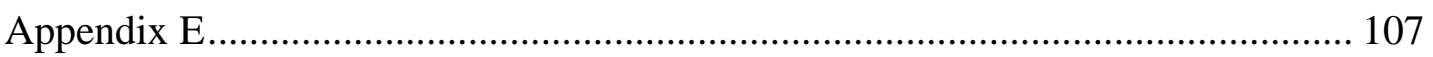




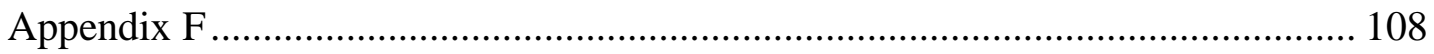

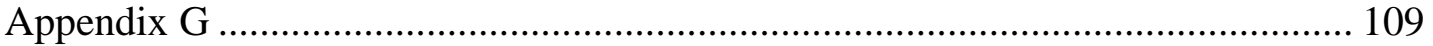




\section{LIST OF TABLES}

Table

Table 2.1: Alzheimer's Disease Brain Observations After Autopsy ............................... 8

Table 2.2: Established Alzheimer's genes and their functional relevance. ....................... 9

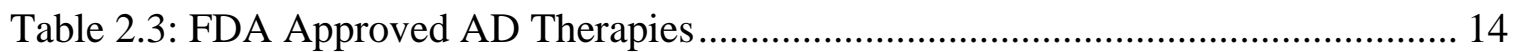

Table 5.1: Summary of P-Value for Maximum and Minimum Magnitudes ................... 38

Table 5.2: Summary of P-Values for Minimum and Maximum Phase .......................... 39

Table 5.3: Summary of P-Values and Confidence Interval for Contrast ........................ 40

Table 5.4: Summary of Correlation P-Values Among AD and NC groups.................... 41

Table 5.5: Summary of Energy P-Values for Both AD and NC groups........................ 41

Table 5.6: Summary of Homogeneity P-Values for AD and NC Groups ...................... 42

Table 5.7: Summary of Entropy P-Values for All AD and NC Groups ........................ 43

Table 5.8: ANN-Backpropagation Structure Results............................................... 45

Table 6.1: Discrete Wavelet Transform training results for both window size ................ 58

Table 6.2: Discrete Wavelet Transform testing results for both window sizes ............... 59

Table 6.3: Fourier transform training results for both window sizes............................. 60

Table 6.4: Fourier Transform testing results for both widow sizes. .............................. 60

Table 6.5: Training results for Gray level co-occurrence matrix (GLCOM), and Gray level co-occurrence matrix (GLCOM) + entropy.....

Table 6.6: Testing results for Gray level co-occurrence matrix

(GLCOM), and Gray level co-occurrence matrix (GLCOM) + entropy......... 62

Table 6.7: Training performance for individual texture features .................................. 63

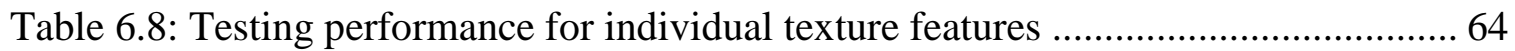

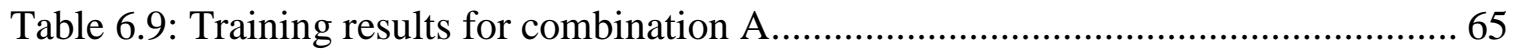

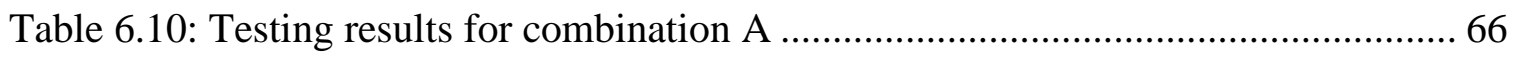

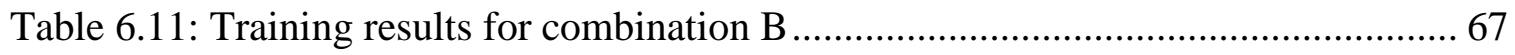

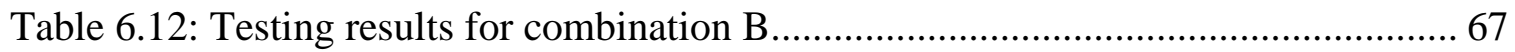

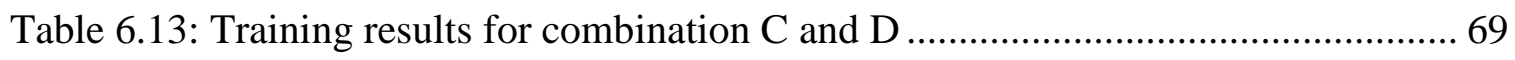

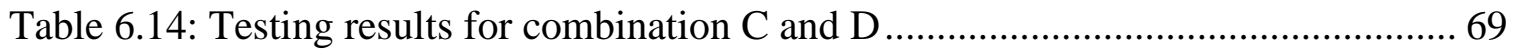

Table 7.1: Training performance of each view's Fourier Transform ............................ 74 
Table 7.2: Testing performance of each view's Fourier Transform .............................. 74

Table 7.3: Training results for all three views using the GLCOM ............................... 75

Table 7.4: Testing results for all three views using the GLCOM ................................. 75

Table 7.5: Training results for all three views using the combination

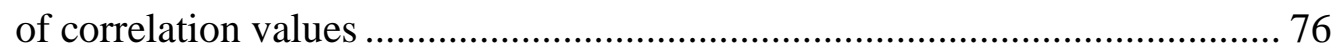

Table 7.6: Training results for all three views using the combination of correlation values.......................................................................... 76

Table 7.7: Training results for combination CII (energy and entropy)............................ 77

Table 7.8: Testing results for combination CII (energy and entropy) ............................ 77

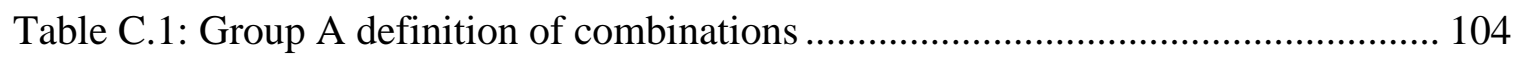

Table C.2: Group B definition of combinations ..................................................... 104

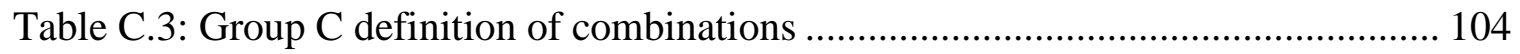

Table C.4: Group D definition of combinations .................................................... 105 


\section{LIST OF FIGURES}

Figure

Page

Figure 1.1: Neuron Comparison in AD .................................................................. 1

Figure 1.2: 10 Early Signs and Symptoms of Alzheimer's........................................ 3

Figure 1.3: Time base diagram the effect of early diagnosis..................................... 5

Figure 2.1: Pathophysiology of Alzheimer's disease. ............................................. 10

Figure 2.2: Decision tree for an individual tested for Alzheimer's disease (AD). .......... 13

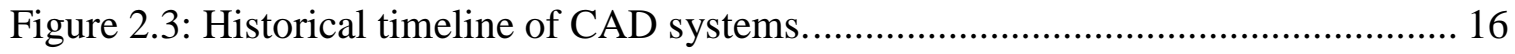

Figure 3.1: Regions of interest and brain comparison among AD and HC ................... 21

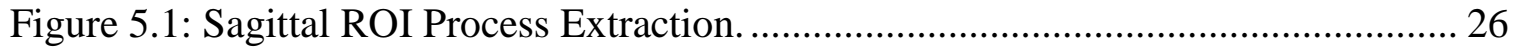

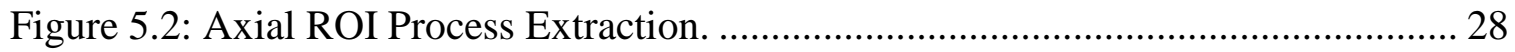

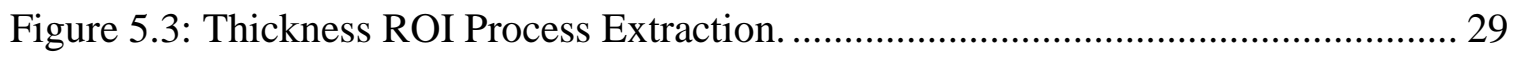

Figure 5.4: Multilayer Feedforward Backpropagation Artificial Neural

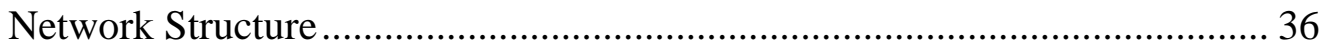

Figure 5.5: MATLAB Neural Network Interface. ................................................... 47

Figure 5.6: Performance Plot for Selected Network................................................. 48

Figure 5.7: Histogram of Networks Performance........................................................ 49

Figure 5.8: Regression Plot at Minimum Gradient of 15 epochs ................................. 50

Figure 7.1: Box Plot of NC and AD subject for each view. ....................................... 78

Figure 8.1: Principal Components plot for Texture features. ....................................... 85

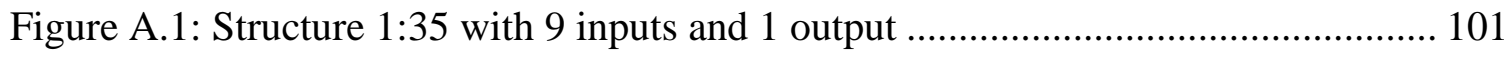

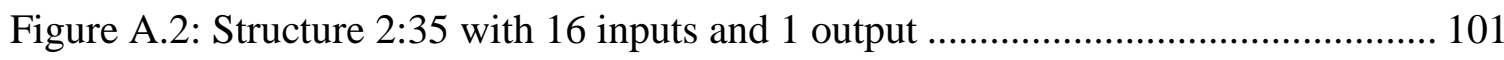

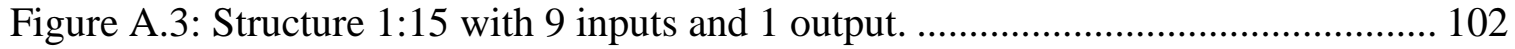

Figure B.1: Structure 1:35 with 8 inputs and 1 output

(combination of any two features except entropy) ................................ 103

Figure G.1: Comparison plot of single view and combination view of CII ................. 109

Figure G.2: Correlation Comparison Plot single view vs. combination view ............... 110 


\section{LIST OF EQUATIONS}

$\begin{array}{ll}\text { Equation } & \text { Page }\end{array}$

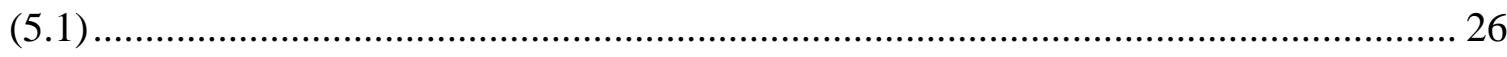

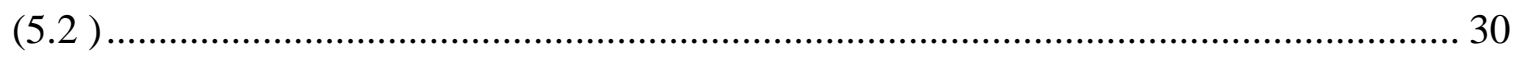

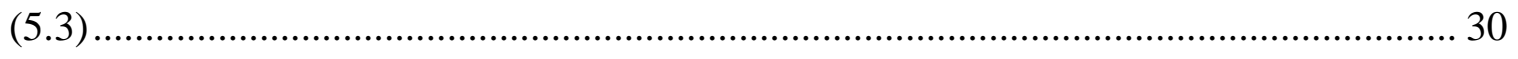

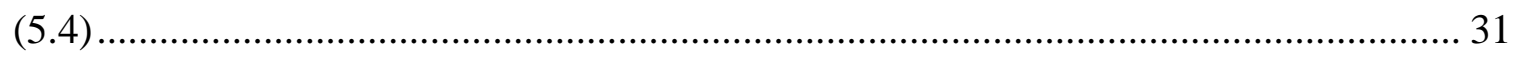

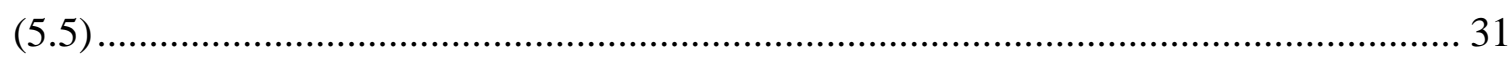

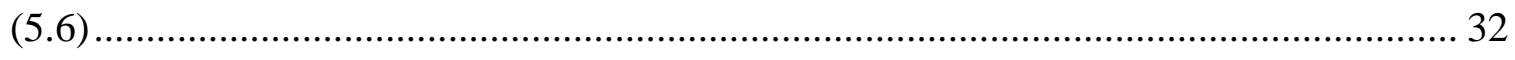

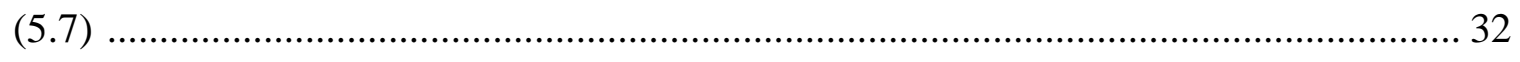

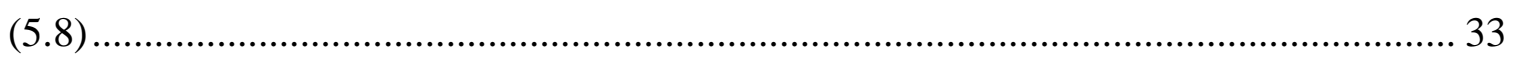

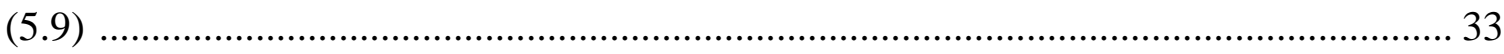

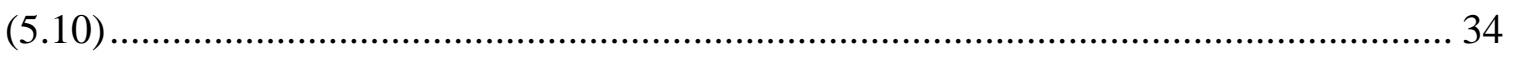

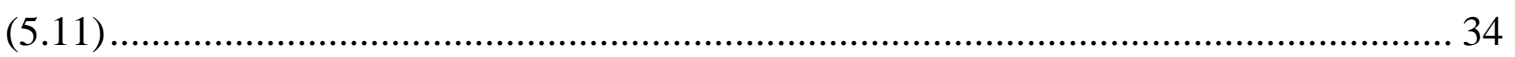

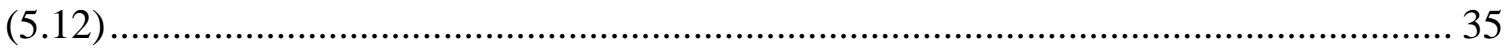

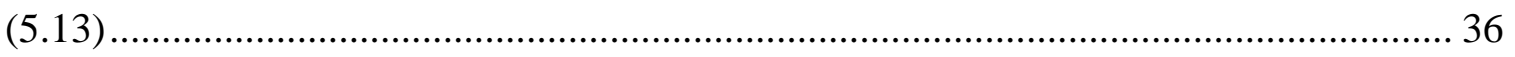

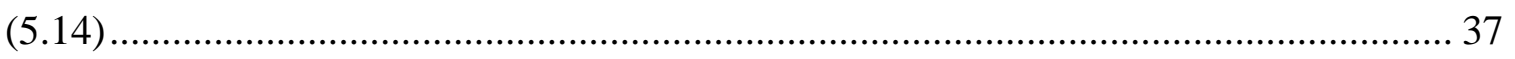

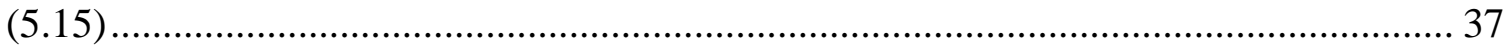

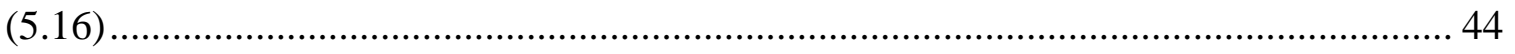

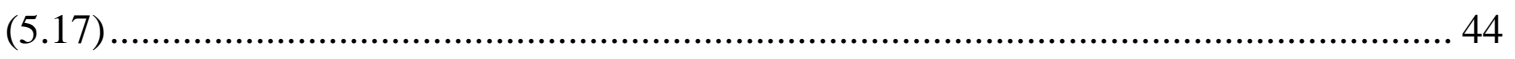

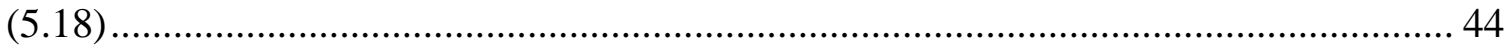

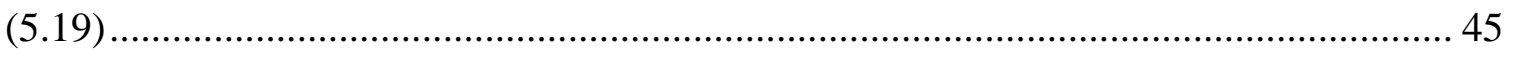

(6.1)

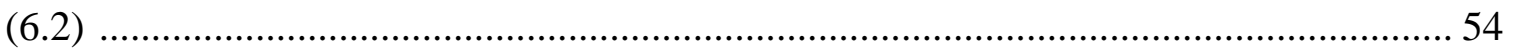

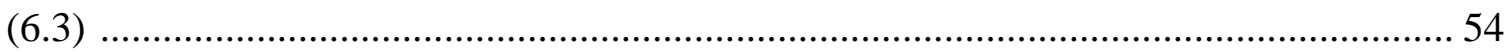




\section{CHAPTER 1: INTRODUCTION}

\subsection{Alzheimer's disease}

\subsubsection{Overview of Alzheimer's disease}

Alzheimer's disease (AD) is known to be the most common form of dementia, comprising 70 percent of all dementia cases [1]. Dementia is a chronic and progressive, irreversible syndrome that deteriorates the cognitive functions. In $\mathrm{AD}$, there is a gradual damage to the neurons which causes them to stop functioning, lose connections, and ultimately die [2], (Figure 1.1).

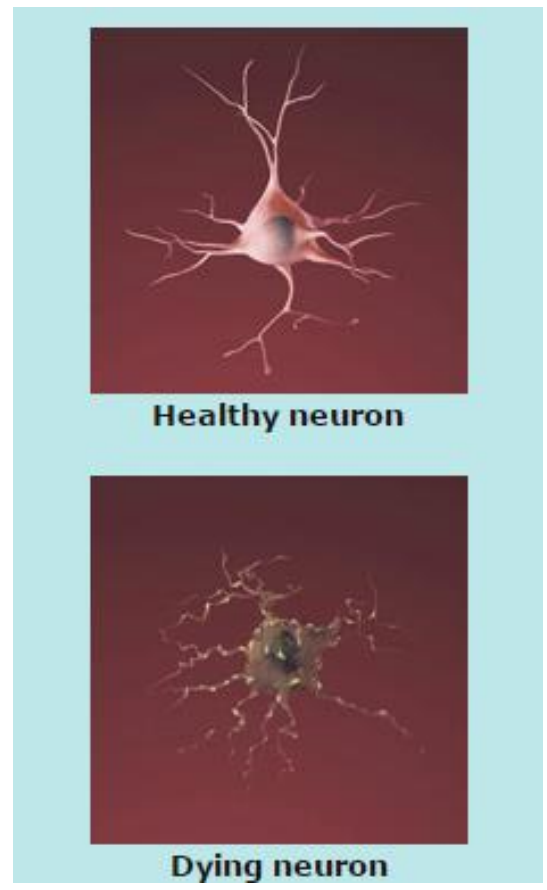

\section{Figure 1.1: Neuron Comparison in AD.}

$\mathrm{AD}$ aggressively destroys neurons and their connections in several regions of the brain related to memory, entorhinal cortex, and hippocampus [2]. Alzheimer's disease damage to the brain is irreversible and extremely severe. The impact of the condition is such that eventually, the individual affected stops performing basic tasks. 


\subsubsection{Symptoms}

Alzheimer's disease studies indicate that symptoms might be part of an already progressing disease and not initial onset. For example, an individual might already have Alzheimer's disease, but it does not show any indicators. Furthermore, the symptoms of $\mathrm{AD}$ can become present one at a time or few at a time; additionally, not all individuals experience the same signs [3].

Even though $\mathrm{AD}$ onset varies from individual to individual, there are common symptoms that can help classify the early and late stages of the disease [3].

Early symptoms: Difficulty remembering names, places, recent conversations, apathy, and depression

Late symptoms: Impaired communication, disorientation, confusion, poor judgment, and behavioral changes.

Acute late symptoms: Difficulty speaking, swallowing, and walking

The difficulty with AD characterization is the overlap of normal aging characteristics and AD symptoms. The difference becomes evident in the progression, re-incidence, and acuteness of the symptom. Ten typical characteristics of aging can become a sign for $\mathrm{AD}$ as they become more acute and severe, (Figure $1.2[4]$ ). 


\begin{tabular}{|c|c|}
\hline $\begin{array}{l}\text { MEMORY LOSS THAT } \\
\text { DISRUPTS DAILY LIFE }\end{array}$ & $\begin{array}{l}\text {-Age-related change: forgetting names or appointment, but remembering } \\
\text { later } \\
\text { •AD sypmtom: Forgetting a recently learned information, asking for the } \\
\text { same information over and over, an icreasing need for memory aids. }\end{array}$ \\
\hline $\begin{array}{r}\text { CHALLENGES IN } \\
\text { PLANNING OR SOLVING } \\
\text { PROBLEMS }\end{array}$ & $\begin{array}{l}\text {-Age-related change: Occasional errors when balancing a checkbook or } \\
\text { dealing with numbers } \\
\text { •AD symptom: Change in the ability to develop and follow a plan, work with } \\
\text { numbers, follow a familiar recipe and difficulty concentrating. }\end{array}$ \\
\hline $\begin{array}{r}\text { DIFFICULTY COMPLETING } \\
\text { FAMILIAR TASKS AT } \\
\text { HOME, AT WORK OR AT } \\
\text { LEISURE }\end{array}$ & $\begin{array}{l}\text {-Age-related change: Occasionally needing help with technology record a } \\
\text { television show. } \\
\text { •AD symptom: Difficulty completing a daily task. Trouble driving to a familiar } \\
\text { location or remembering the rules of a favorite game. }\end{array}$ \\
\hline $\begin{array}{r}\text { CONFUSION WITH TIME } \\
\text { OR PLACE }\end{array}$ & $\begin{array}{l}\text {-Age-related change: Getting confused about the day of the week, but } \\
\text { figuring it out later } \\
\text {-AD symptom: Lose track of dates, seasons and the passing of time. }\end{array}$ \\
\hline $\begin{array}{r}\text { TROUBLE } \\
\text { UNDERSTANDING VISUAL } \\
\text { IMAGES AND SPATIAL } \\
\text { RELATIONSHIPS }\end{array}$ & $\begin{array}{l}\text {-Age-related change: Vision changes due to cataracts or other eye problems } \\
\text { •AD symptom: Difficulty reading, judging distances, and determining color or } \\
\text { contrast }\end{array}$ \\
\hline $\begin{array}{r}\text { NEW PROBLEMS WITH } \\
\text { WORDS IN SPEAKING OR } \\
\text { WRITING }\end{array}$ & $\begin{array}{l}\text {-Age-related symptom: Having trouble finding the right word } \\
\text { •AD symptoms: Trouble following or joining a conversation. Stop in the } \\
\text { middle of a conversation or repeat themselves over and over. }\end{array}$ \\
\hline $\begin{array}{r}\text { MISPLACING THINGS AND } \\
\text { LOSING THE ABILITY TO } \\
\text { RETRACE STEPS }\end{array}$ & $\begin{array}{l}\text {-Age-related change: Misplacing things from time to time and retracing steps } \\
\text { to find them } \\
\text { •AD symptom: Put things in unusual places. Unable to retrace their steps to } \\
\text { track misplaced items. }\end{array}$ \\
\hline $\begin{array}{r}\text { DECREASED OR POOR } \\
\text { JUDGMENT }\end{array}$ & $\begin{array}{l}\text {-Age-related change: Making a bad decision once in a while } \\
\text { •AD symptom: Changes in judgment or decision-making. Lack of self- } \\
\text { grooming. }\end{array}$ \\
\hline $\begin{array}{r}\text { WITHDRAWAL FROM } \\
\text { WORK OR SOCIAL } \\
\text { ACTIVITIES }\end{array}$ & $\begin{array}{l}\text {-Age-related change: Sometimes feeling weary of work, family or apathetic } \\
\text { to social obligations } \\
\text { •AD symptom: Forgetting how to complete a hobby, or play a favorite sport, } \\
\text { and ultimately removing themselves from social activities, work projects or } \\
\text { sports. }\end{array}$ \\
\hline $\begin{array}{r}\text { CHANGES IN MOOD AND } \\
\text { PERSONALITY }\end{array}$ & $\begin{array}{l}\text {-Age-related change: Developing a specific way of doing things and become } \\
\text { irritable when a routine is disrupted. } \\
\text { •AD symptom: Mood and personality changes, becoming confused, } \\
\text { suspicious, depress, fearful or anxious. }\end{array}$ \\
\hline
\end{tabular}

Figure 1.2: 10 Early Signs and Symptoms of Alzheimer's.

AD symptoms have an overlap with age-related changes. However, AD symptoms are severe, permanent, frequent, and ultimately alter the overall behavior and mood of the individual [4]. 


\subsubsection{Alzheimer's Diagnosis}

The characteristics of Alzheimer's disease, aging changes, and other forms of dementia are highly similar that making a proper diagnosis becomes complex. Thus, as with any other medical condition, a proper diagnosis can lead to a more successful treatment. However, currently, there are not reliable tests that can definitively diagnose $\mathrm{AD}$ in living individuals [5].

\subsubsection{Computer Aided Diagnostic (CAD) systems}

In a medical setting, human error becomes a limiting factor for proper diagnosis due to the complexity of the human eye-brain system, the training, and the experience of the diagnostician. Additionally, some situations can further influence a diagnosis such as physical fatigue, distractions, and biases. Consequently, the introduction of image enhancement or medical imaging has emerged, and even though extremely useful, it cannot aid with fatigue or distractions [6]. For many conditions, a proper and early diagnosis can be the difference between life and death; therefore, a tool that can minimize human error and aid diagnosis, is nothing less than essential [7]. Computer Aided Diagnosis (CAD) systems aim to enhance current diagnostic methods.

\subsection{Project Justification}

\subsubsection{Overview}

Official death certificates of 2013 reported 84,767 deaths from Alzheimer's disease, making it the sixth-leading cause of death in the United States. The rate of AD is estimated to double by 2050 due to the large number of aging individuals especially in United States, China, and India [8] [9]. Moreover, the current cost associated with AD 
will also increase from $\$ 100$ billion to a $\$ 116$ billion per year [10]. The neurodegeneration of $\mathrm{AD}$ occurs decades before symptoms of dementia are evident. PET and MRI images of individuals between 20-39 years of age with AD biomarkers presented abnormally low rates of glucose metabolism in the areas known to be affected by AD (parietal, temporal, and prefrontal cortex) [11]. Therefore, having an efficient methodology for the early and proper diagnosis can lead to better treatments, (Figure 1.3). However, the issue is in the method for a non-invasive, proper, and early diagnosis. Due to the pathological overlapping of the clinical symptoms of dementia, many individuals can be misdiagnosed, leading to the use of wrong medications with unknown health consequences. A report suggested that the number of people giving inappropriate medication can be as high as 67.10 percent [12].

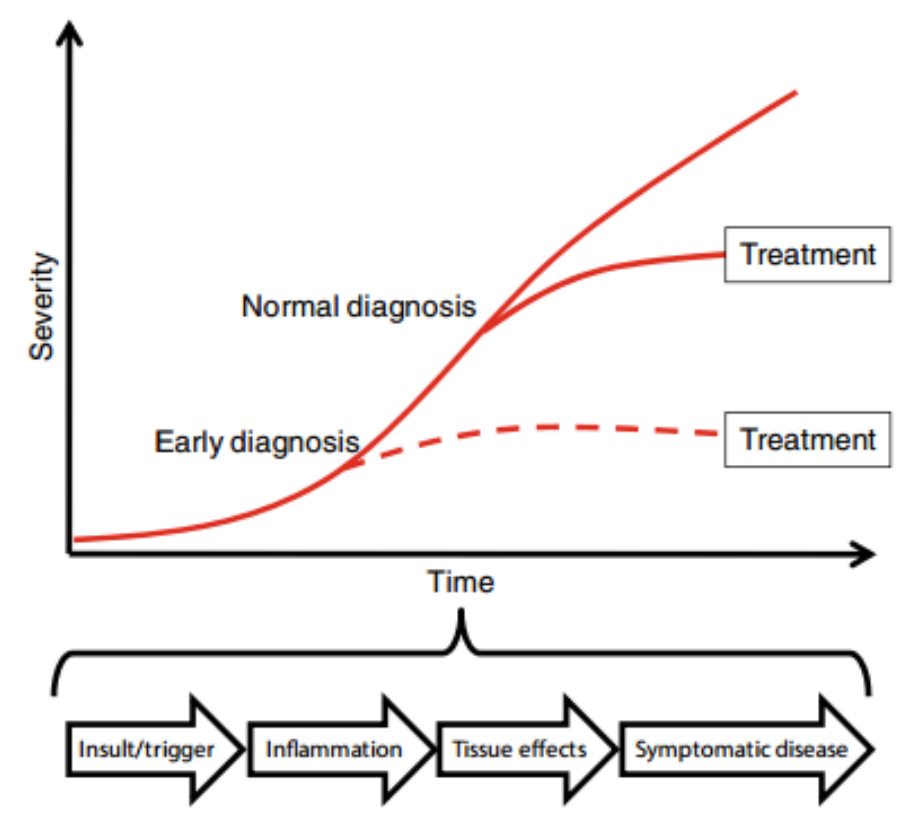

Figure 1.3: Time base diagram the effect of early diagnosis.

Early diagnosis combined with effective treatment can improve individual's overall quality of life [13]. An early and accurate diagnosis of $\mathrm{AD}$, even before clinical 
symptoms appeared can aid individuals to take preventive measures before they lose the ability to do so [14] [15].

\subsubsection{Specific Aims}

The principle questions to be investigated through this thesis are as follows:

- Aim 1: Investigate if texture image attributes and frequencies domains attributes from MRI images contain valid information which can serve as classification attributes, and therefore aid for the classification of Alzheimer's disease.

- Aim 2: Investigate if one view of an MRI image gives comparable results vs. using a combination of three views (axial, coronal, and sagittal).

- Aim 3: Investigate if the combination of a feedforward artificial neural network, texture and frequency attributes from MRI images achieve the same level of performance as the clinicians 


\subsubsection{Project Goal}

Computer aided diagnosis (CAD) systems are part of a larger number of efforts being made to identify the proper approach for machine learning programming. An Artificial Neural Network (ANN) system which utilizes image processing techniques can offer an alternative approach to the current CAD systems. Additionally, there is no consensus on the best image attributes for the identification of AD. The goal of this thesis is to investigate if the use of MRI images, specifically texture and frequency attributes in combination with a feedforward artificial neural network, can lead to the classification of individuals with Alzheimer's in comparison with the clinician's performance. 


\section{CHAPTER 2: LITERATURE REVIEW}

\subsection{Overview of AD}

\subsubsection{Etiology}

A 51-year-old female patient that presented hallucination, language deficit, cognitive deficient, and memory loss was the first diagnosis of the neurodegenerative disease. After the patient's death in 1901, Dr. Alois Alzheimer performed microscopic analyses of her brain observing that there were structures identified as plaques and tangles. He also identified atherosclerotic changes (built up of plaque) in the large cerebral arteries matching several observations of other neurologists which performed similar observations in other patients [16]. These similar observations are among the most obvious abnormalities found in deceased individuals whose brains were microscopically analyzed, (Table 2.1).

Table 2.1: Alzheimer's Disease Brain Observations After Autopsy

\begin{tabular}{|l|l|l|}
\hline Abnormality & Location & Description \\
\hline Amyloid Plaques & Spaces between neurons & $\begin{array}{l}\text { Plaques are in its majority } \\
\text { formed by abnormal deposits } \\
\text { of beta-amyloid. }\end{array}$ \\
\hline Neurofibrillary tangles & Inside the neurons & Abnormal tau protein clumps \\
\hline coss of neural & Axon & $\begin{array}{l}\text { Synaptic connections failure } \\
\text { leads to degeneration of the } \\
\text { brain, and ultimately dead }\end{array}$ \\
\hline
\end{tabular}

\subsubsection{Prevalence and Risk Factors}

Age influences AD prevalence. Individuals between ages 60-64 have about 1 percent occurrence of the disease while individuals 85 years and older have a higher 
prevalence of 33 percent [17]. Even though aging plays a role in the onset and progression of $\mathrm{AD}$, the syndrome is a complex multifactorial disease influenced by many other elements including environmental and genetic factors.

\subsubsection{Genetic Factors}

Some genetic factors increase the risk for AD. The genetics of AD show that the syndrome can be familial and sporadic. The autosomal inheritance of the disease occurs in less than 2 percent of $\mathrm{AD}$ individuals. The autosomal $\mathrm{AD}$ is present in an early onset and associated with specific genes. (Table 2.2). The clear majority have a sporadic and late-onset of $\mathrm{AD}$ accounting for 90-95 percent of the cases [18].

Table 2.2: Established Alzheimer's genes and their functional relevance

\begin{tabular}{l|l|l|l}
\hline Gene (protein) & Chromosome & Inheritance & Role in AD pathogenesis \\
\hline $\begin{array}{l}\text { APP (amyloid precursor } \\
\text { protein) }\end{array}$ & 21 & Autosomal dominant & $\begin{array}{l}\text { Mutations in the APP gene } \\
\text { promote cleavage at b or g sites } \\
\text { leading to overproduction of A } \beta\end{array}$ \\
\hline PSEN1 (presenilin 1) & 14 & Autosomal dominant & $\begin{array}{l}\text { Mutations in the PS1 gene promote } \\
\text { cleavage at the g site leading to } \\
\text { overproduction of A } \beta\end{array}$ \\
\hline PSEN2 (presenilin 2) & 1 & Autosomal dominant & $\begin{array}{l}\text { Mutations in the PS2 gene promote } \\
\text { cleavage at the g site leading to } \\
\text { overproduction of A } \beta\end{array}$ \\
\hline APOE (apolipoprotein E) & 19 & Modifies age of onset & Promotes the deposition of A $\beta ?$ \\
\hline
\end{tabular}

PS1 is the most frequent form of the mutation while APP and PS2 are less common on their impact in the beta-amyloid protein. The $A \beta$ is one of the principal components of senile plaques. The apolipoprotein E gene (APOE) is identified as a major risk-factor for late-onset $\mathrm{AD}[19]$.

Although the causes of sporadic forms of $\mathrm{AD}$ are not entirely understood, it is evident that with aging there are interactions between genetics and the environment that contribute to the onset. The primary genetic risk factor in the overall population is the 
ApoEs4 allele which increases the accumulation of the amyloid-beta (A $\beta)$ [18]. A significant decrease of $\beta$-amyloid - $-42_{2}$ levels and an increase of tau proteins (p-Tau) lead to the formation of fibril tangles and the $A \beta$ plaque that in turn resulted in neuron necrosis [20], ( Figure 2.1).

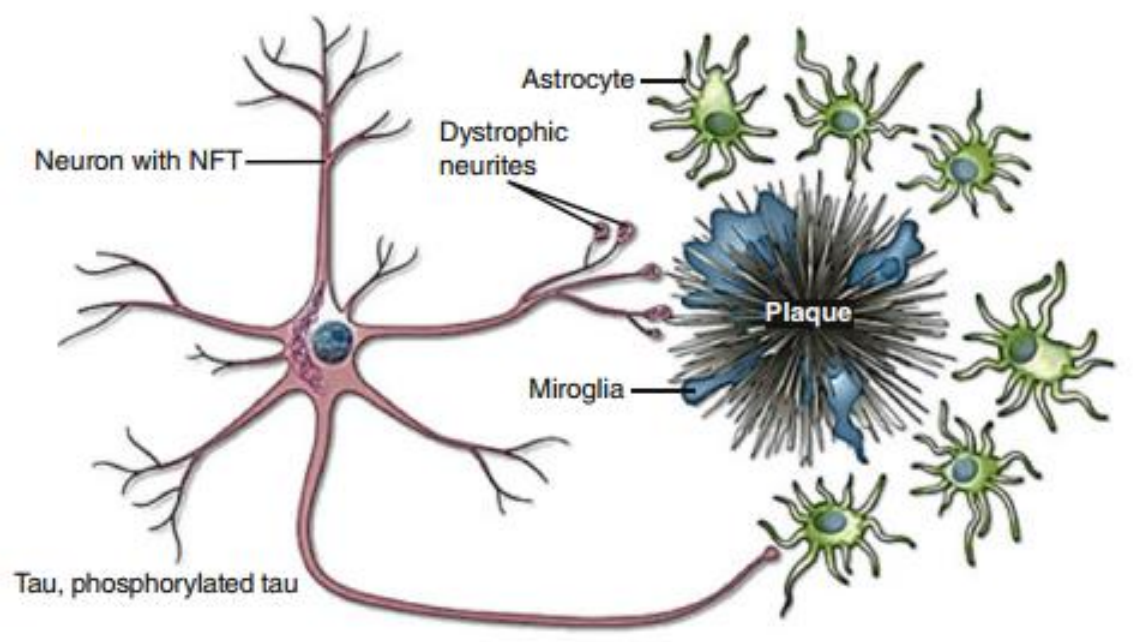

Figure 2.1: Pathophysiology of Alzheimer's disease.

Low levels of $\beta$-amyloid 1-42 $_{2}$ lead to amyloid accumulation into extracellular plaques. Increase of p-Tau leads to intra-neurofibrillary tangles (NFT) [21]

\subsubsection{Aging}

The highest factor associated with Alzheimer's disease is aging. Most individuals with $\mathrm{AD}$ are people 65 years or older. About 44 percent of $\mathrm{AD}$ individuals are 75-84 years old, and about 15 percent are 65-74 years of age [3]. However, not all aging individuals are known to develop AD or other dementias. 


\subsubsection{Additional factors}

Other factors are indirectly related to $\mathrm{AD}$, which related to increasing other health conditions due to lifestyle. Mild cognitive impairment (MCI) individuals have a higher risk of developing Alzheimer's disease. Moreover, vascular health issues such as diabetes mellitus, raised blood pressure, obesity, hyperlipidemia among others cardio vascular diseases make vascular health a key factor underlying age-related cognitive decline [22]. Thus, is concluded that vascular health can influence the onset of AD. Consequently, Regular exercise, an active way of life, and a balanced diet can reduce the risk of vascular disorders and consequently the risk for $\mathrm{AD}[23]$.

\subsubsection{Diagnosis Methods}

\subsubsection{Background}

The characteristics of Alzheimer's disease, aging changes, and other forms of dementia are highly similar that making a proper diagnosis becomes complex. Thus, as with any other medical condition, a proper diagnosis can lead to a more successful treatment. However, currently, there are not reliable tests that can definitively diagnose $\mathrm{AD}$ in living individuals [5]. The current diagnosis of $\mathrm{AD}$ follows a specific clinical diagnosis criteria established in 1984. The National Institute of Neurological and Communicative Disorder and Stroke (NINCDS) and the Alzheimer's Disease and Related Disorders Association (ADRDA) known as the NINCDS-ADRDA Alzheimer's criteria in combination with the Statistical Manual for Mental Disorders (DSM-IV-TR) were the original bodies that introduce the guide for AD diagnosis [24].

The NINCDS-ADRDA provides a table for the PROBABLE, POSSIBLE, and DEFINITE Alzheimer's disease diagnosis. The diagnosis is a multi-step process where 
the first step is in identifying whether the patient has dementia or not. If dementia is present, then an assessment of what type of dementia the individual has is performed. An $\mathrm{AD}$ diagnosis focuses on identifying a memory disorder and a deficiency of at least one other cognitive area or a combination of physical deterioration [24]. Additionally, family history and laboratory results from a lumbar puncture, and Electroencephalogram (EEG) or a computerized tomography (CT) are also part of the assessment [25]. However, a major limitation of the current diagnostic methods is that A DEFINITIVE diagnosis can only occur after a brain biopsy or an autopsy.

\subsubsection{Recent Diagnosis Modifications}

Within the last few years, the clinical diagnosis of Alzheimer's disease has extended to include magnetic resonance imaging (MRI), and positron-emission tomography (PET) [2].( Figure 2.2). AD is a complex problem because of its diverse range of symptoms and because of its overlap with other conditions. Two agencies, The National Institute on Aging and the Alzheimer's Associations, are working together to revise the Diagnosis criteria after 27 years [26]. The newly proposed clinical criterion for AD diagnosis has two major groups: clinical settings core criteria and research purpose. The clinical settings are an extension of the 1984 guidelines, but more comprehensive and sensitive to other conditions. The research purpose follows the clinical criteria but includes biomarkers for definitive diagnosis. 


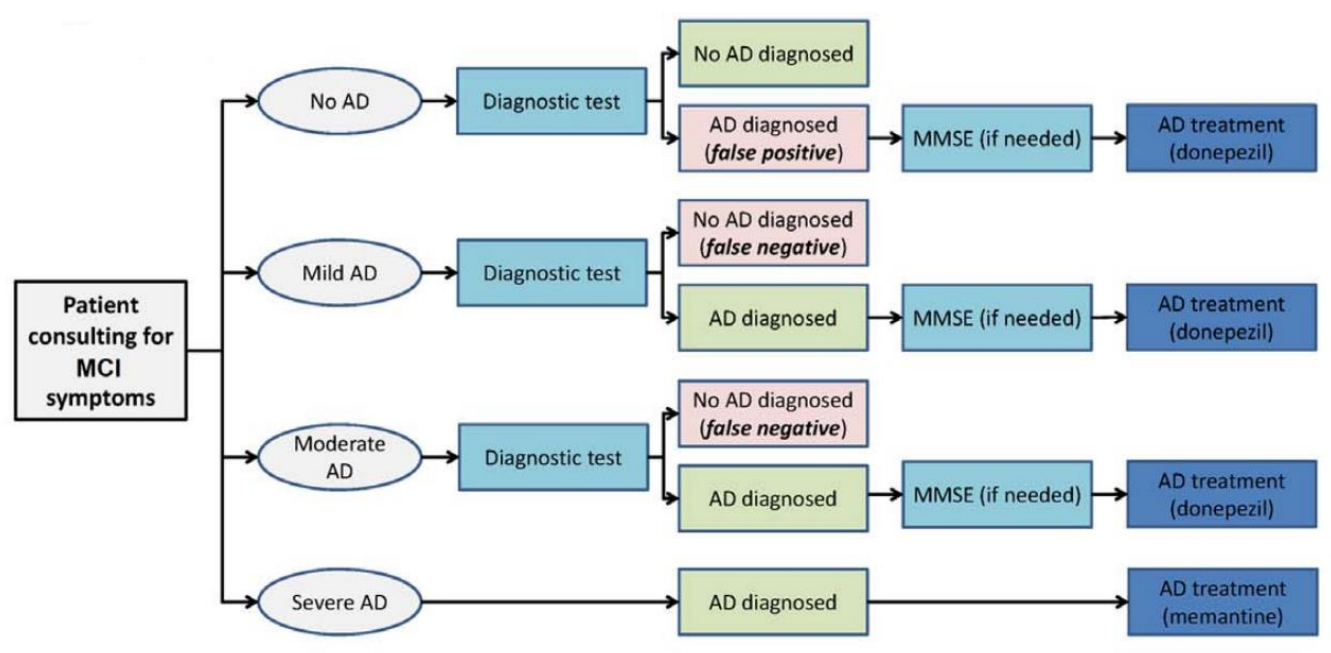

Figure 2.2: Decision tree for an individual tested for Alzheimer's disease (AD).

Mild Cognitive Impairment (MCI) is linked to 50 to 70 percent of AD cases [13]. Even though MCI is a cognitive decline, its symptoms are not severe enough to interfere with daily life; however, 15 percent of MCI individuals develop dementia within a year and 80 percent within 6 years. Additionally, MCI is not isolated to a risk of AD only, but to other dementias like Parkinson's disease (PD) [13]. California has developed a classification guide that is consolidates the general symptoms of AD [27]

\subsubsection{Imaging as Diagnostic Tools}

\subsubsection{MRI Imaging and Its Limitations}

The early changes in the brain in living subjects which can improve diagnostic tools with higher sensitivity and specificity are observed by neuroimaging techniques like magnetic resonance imaging (MRI) [28]. Currently, it is recommended for European nations to perform a neuroimaging study to rule out reversible dementia or other causes for the symptoms. MRI's high resolution and tissue contrast are used for the assessment of cerebral atrophies, and other conditions such as the case of small vessel ischemic 
disease, white matter sensitivity, medial temporal atrophy, and global cortical atrophy $[26,27]$

\subsubsection{Current Treatments}

Since the beginning of last century, Alzheimer's disease still is an incurable condition. Currently there are therapies that aid with symptomatic improvements of some $\mathrm{AD}$; however, there is no evidence that such therapies slow or stop the progression of the disease [26]. There are a total of five FDA-approved drugs to treat Alzheimer's disease, (Table 2.3)

Table 2.3: FDA Approved AD Therapies

\begin{tabular}{|l|l|l|c|}
\hline Drug name & Brand name & Approved For & FDA Approved \\
\hline 1. donepezil & Aricept & All stages & 1996 \\
\hline 2. galantamine & Razadyne & Mild to moderate & 2001 \\
\hline 3. memantine & Namenda & Moderate to severe & 2003 \\
\hline 4. rivastigmine & Exelon & All stages & 2000 \\
\hline $\begin{array}{l}\text { 5. donepezil and } \\
\text { memantine }\end{array}$ & Namzaric & Moderate to severe & 2014 \\
\hline
\end{tabular}

The U.S Food and Drug Administration (FDA) approve the donepezil in 2014, but the studies for approval took place at the beginning of 2000 [3]. The donepezil and memantine were originally intended for the treatment of mild-to-moderate AD; however, in 2012 studies show that this treatment can benefit moderate to severe AD individuals. 


\subsection{Computer Aided Diagnosis (CAD) Systems}

\subsubsection{Overview}

Medical images are by far one of the best diagnostic tools in the market today; however, one significant limitation is human error. Even though physicians and radiologist have formal training, the level of training, the experience, the methodology, and other factors such as distractions, fatigue, and environment, can affect the outcome of

the diagnosis [6]. Thus, CAD systems can minimize diagnostic errors, and provide a confirmation of the diagnosis to the radiologist. However, CAD systems performance depends on the type of machine learning technique applied to it.

\subsubsection{Background}

In the mid-1950s the idea of using an automated diagnosis computer in radiographs sparked. In 1967, Fred Winsberg described the first CAD system for mammograms [29]. However, CAD systems extensive and severe research begun in the 1980s. Today in the United States, they form part of most routine clinical work to detect breast cancer with the use of mammograms [29], (Figure 2.3). Before the 1980s the concept of automated diagnosis or automated computer diagnosis originated, but in the 1980's the current concept of CAD systems changed the scope, expectations, and role of the computer in diagnosis. 


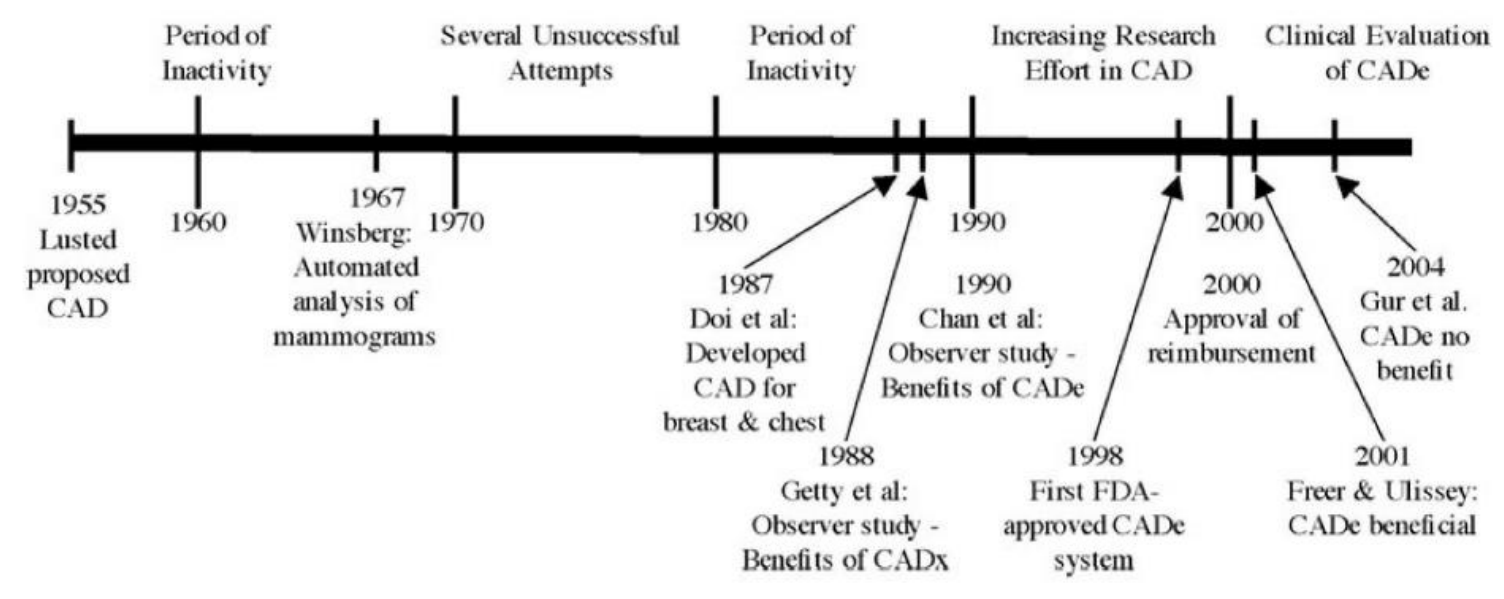

Figure 2.3: Historical timeline of CAD systems.

The early studies of the computer-aided diagnostic systems began in late 1950. In 1988, the CAD systems' research gained force, and the philosophy of CAD systems changed [29].

\subsubsection{Automated Computer Diagnosis and CAD}

The main similarities between both systems are the quantitative analysis of digital medical images, and that both require a high sensitivity and high specificity [6]. The difference between the systems depends on the role of the diagnostician. Automated computer diagnosis's core idea is the use of a computer to replace the physician or radiologist. Consequently, automated computer diagnosis is the primary source of diagnosis, and its performance is equal to the performance achieved by the computer.

On the other hand, CAD systems use the computer's output to aid the diagnostician rather than to replace the physician. CAD systems are a diagnosis confirmation, and their performance level is equal to the performance achieved by the expert [6]. In conclusion, automated computer diagnosis systems seek to replace the 
physician in its role of diagnostician, while CAD systems aim to improve diagnosticians' ability to detect abnormalities as discussed later on the text.

\subsubsection{CAD system}

The two categories of CAD systems depends on the function of the computer. Computer-aided detection (CADe) systems are used to mark abnormal regions in an image that the clinician can use for diagnosis. Computer-aided diagnosis (CADx) systems provide the clinician an assessment of a condition such as giving disease type, stage, the probability of progression or the severity of illness [30].

\subsubsection{CAD systems performance}

Computer-aided diagnosis (CAD) systems can aid physicians, provide a second "expert" opinion, and ultimately decrease the health cost and misdiagnosis rate [31]. In the case of MRI images for breast cancer diagnosis, the CAD system resulted in a higher sensitivity (97.9 percent) and specificity (86.4 percent) while compared to two expert breast cancer radiologists. The mean values for the radiologist were 84.6 percent for sensitivity and 68.8 percent for specificity [32]. Similar results were obtained for other conditions such as coronary artery which the CAD system gives an accuracy of 98.5 percent (higher than the gold standard of 97.32 percent). In the diagnosis of gliomas

with MRI images the CAD system achieved an accuracy of 87 percent, a sensitivity of 79 percent and a specificity of 90 percent. The radiologist results were lower with an 81 percent for accuracy, 87 percent for sensitivity, and 84 percent for specificity [33]. These studies demonstrated that CAD systems accurate predictions can improve the diagnostician's levels of performance. 


\subsubsection{Artificial Neural Network}

CAD systems use data and machine learning techniques to assist with the diagnosis of several conditions. Commonly the systems include segmentation, feature extraction, and classification [34]. In the case of $\mathrm{AD}$, there have been a significant number of classifiers used such as Random Forest which has good accuracy and robustness, but it presents bias towards multi-categorical features [35]. The most common classifier in literature applied to Alzheimer's disease diagnosis is the Support Vector Machine (SVM) or a deviation of it. This algorithm is a supervised classifier that uses pattern recognition to predict the category of the sample provided. The major shortcomings of SVM are the large number of features required for classification because those can lead to over computer power, and computational complexity [36]. Moreover, a high number of input features relative to the number of subjects can inquire issues such as dimensionality and large hypothesis space [37].

Artificial Neural Networks (ANN) had been useful for image classification where the complexity of the data is high, and where the input and output have a nonlinear relationship. In the comparison of brain aging, the implemented ANN model achieved superior prediction accuracy than the manifold learning combined with SVM. Additionally, in the comparison of wavelet classifications (using Discrete Wavelet Transform from MRI images), ANNs have outperformed SVM achieving 100 percent accuracy [38]. The limitations of the ANN models are the identification of the neural network structure. 


\subsubsection{Feature identification}

There are several approaches to identification of abnormalities in the diagnosis of AD. Most are related to specific Regions of Interest (RIO'S) the issue with those are the process of extraction, and the features used for analysis [39]. Another approach has been the comparison of white matter volume and white matter lesions which are also localize regions; however, the limitation is that usually matter lesions are present on later stages and not on all the patients. There is evidence that supports that brain morphology changes with $\mathrm{AD}$, and that there is low-frequency fluctuations also associated with the disease [40]. 


\section{CHAPTER 3: PROJECT APPROACH}

\subsection{Background}

\subsubsection{ADNI database}

Due to the limitations of acquiring MRI images, this study used the MRI images from the ADNI database. The Alzheimer's Disease Neuroimaging Initiative (ADNI) is a global cooperative research grant whose main objective is to use clinical, imaging, and genetic biomarkers for the identification AD. The database provides data for Alzheimer's disease (AD) subjects, Mild Cognitive Impairment (MCI) subjects, and Normal Control subjects (NC) [41].

\subsubsection{MATLAB neural network toolbox}

MATLAB is a high-performance language used for diverse fields, and it integrates computation, visualization, and programming to express the solution in a mathematical form. The Neural Network Toolbox is used to model complex nonlinear systems that are not able to be modeled with a closed-form equation. The Neural Network Toolbox supports ANN and other dynamic networks [42].

\subsubsection{Brain regions}

MRI technology depends on the changes in energy states of protons, which result in radio-frequency signals. A combination of a different number of pulses can become sensitive to different tissue characteristics. Thus, MRI images can aid to assess atrophy and changes in tissue [43]. The hippocampus is one of the tissue areas known to be affected in the earliest stages of $\mathrm{AD}$. The hippocampus or hippocampal formation is in general applied to the dentate gyrus (DG) [44]. Also, AD is associated with the decrease 
in brain mass, which leads to larger ventricles and a shrinkage of the cerebral cortex [3], (Figure 3.1 ).
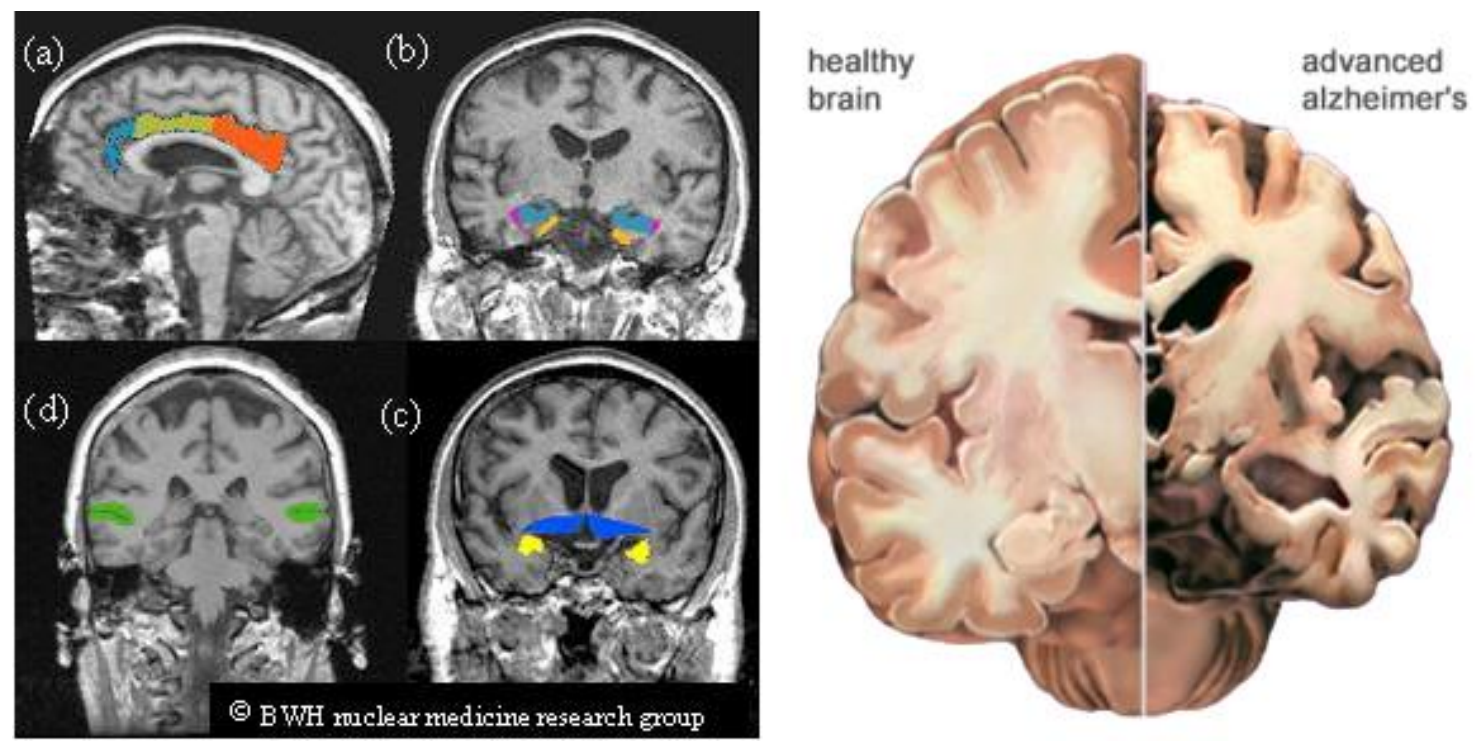

Figure 3.1: Regions of interest and brain comparison among AD and HC.

The left images are color coded to show different regions: blue is used to indicate the rostral anterior cingulate, green indicates the caudal anterior cingulate. Red is associated with the posterior cingulate. The image of interest is image $b$ which shows the hippocampus with a blue collar and the entorhinal cortex with orange. [44]. The right image compares a healthy control brain (left side) against an advanced AD brain. The images show a difference in the brain size, ventricles volume and cortex thickness [3].

\subsection{Approach Overview}

The MRI of the same number of healthy controls and AD subjects will be obtained from the ADNI database. The MRI images will be processed, and regions of interest (ROIs) will be identified for feature extraction through image processing techniques. The 
results of the feature extraction will be classified utilizing a feedforwards backpropagation neural network, which is a specific structure of feedforward artificial neural network. Additionally, the neural network toolbox form Matlab, which is an integrated program to run neural networks would be used for the programing portion of the project. The neural networks ideal structure will be identified in terms of hidden layers and neurons in each layer. The results of classification and the features will be examined. 


\section{CHAPTER 4: MATERIALS}

\subsection{MRI Images}

All ADNI subjects underwent a pre-screening process which included several neuropsychological examinations. The results are associated with specific clinical characteristics that aid diagnosis. Some of the examinations include the Clinical Dementia Ratio (CDR) and Mini-Mental State Examination (MMSE score). The MRI images for both Alzheimer's disease (AD) subjects and Normal control (NC) subjects were acquired using the ADNI only database with a 3 Tesla and a T1-weighted using the SAGITAL Siemens scanner. Additionally, the acquisition was a 3D type with a Pa Coil, and a 9-degree flip angle, $[240,256,176]$ pixel matrix and a $1.2 \mathrm{~mm}$ slice thickness.

\subsection{Subjects data}

\subsubsection{Normal control (NC) subjects and Alzheimer's disease (AD) subjects}

An advance search was conducted to select specific subject characteristics to allow for a balance data acquisition. The pilot study had fewer subjects than stage two and stage three. The demographics are discussed within each stage. The NC group MMSE scores range from 27 to 33, and all NC subjects had a CDR score of zero for all stages. For the AD group, the MMSE scores for these subjects range from 8 to 29, and their CDR scores ranged from .3 to 2 , which was the same for all three stages.

\subsection{The Software and hardware}

The Mathworks student edition package with the neural network and image processing toolbox was utilized for all programming and image processing. Adobe Photoshop was used to save the MRIs to a *.png and to ensure all images had the same 
dimensions and pixel ratio. A Lenovo laptop idea pad with an i7 core processor was used for all the data and image processing.

\subsection{MRI acquisition}

The MRI images obtained were 3D MPRAGE T1-weighted. These types of images are achieved by using a short Time to Echo (TE) and short Repetition Time (TR). Additionally, all the images were corrected by using Gradwarp, B1 non-uniformity, and N3 correction. The Gradwarp allows for the correction of geometry distortion due to gradient non-linearity. B1 non-uniformity is performed during acquisition to correct any non-uniform intensity results. N3 employs a sharpening of the image utilizing histogram equalization. 


\section{CHAPTER 5: STAGE ONE: PILOT STUDY}

\subsection{Materials for pilot study}

\subsection{Sample size and demographics}

There were a total of 212 images used, which were equally distributed: 106 for NC, and 106 for the AD group. For the NC group the females' age ranges from 58 to 89 years with a mean of 73.5 years, while the male subjects had a range of 61 to 90 years and a mean of 75 years. The AD group age range for the females was 58 to 93 years and the males' age range was 56 to 88 years with a mean of 72 years.

\subsection{Methods}

\subsubsection{Image pre-processing}

There were 212 sagittal view images and 212 axials view images used for the analysis. The selected brain regions for both sagittal and axial views linked with specific changes consequence of $\mathrm{AD}$ presence were selected. Furthermore, there were two main regions selected from the axial images which are referred as thickness view images.

\subsubsection{Sagittal images}

Several structural imaging studies have identified the hippocampus as a diagnostic marker for $\mathrm{AD}$ because both the hippocampus and thalamus are associated with the decrease of volume matter evident of $\mathrm{AD}$ [45]. Furthermore, the structural studies showed that the hippocampus is reduced by about $15-25$ percent volume in individuals mildly affected by AD [45]. Thus, these two areas were identified as regions of interest (ROIs). The ROIs were extracted from the sagittal images through the process of convolution, (Equation 5.1). 


$$
g(x, y)=h(x, y) * f(x, y)
$$

Where $\mathrm{g}(\mathrm{x}, \mathrm{y})$ is the enhance image (region of interest), $\mathrm{h}(\mathrm{x}, \mathrm{y})$ is the mask (binary image), and $f(x, y)$ is the original image [39]. An NC MRI was used as a baseline image. The baseline was converted from a three-color array to a two-color array, and then the ROI was selected as a single region, (Figure 5.1). Then the original image was changed from a gray color image to a binary image. The original image was converted to a binary image as the mask finds isolated points corresponding to the region. Both images were multiplied together (convoluted) resulting in the ROIs. When a satisfactory resulted image was achieved, then the mask was used to process the rest of the images, (Figure 5.1). A function was created for extraction and used on all images simultaneously. Utilizing one mask for all images allows for a standardized size of the ROIs, so all values extracted from the feature have the same parameters.
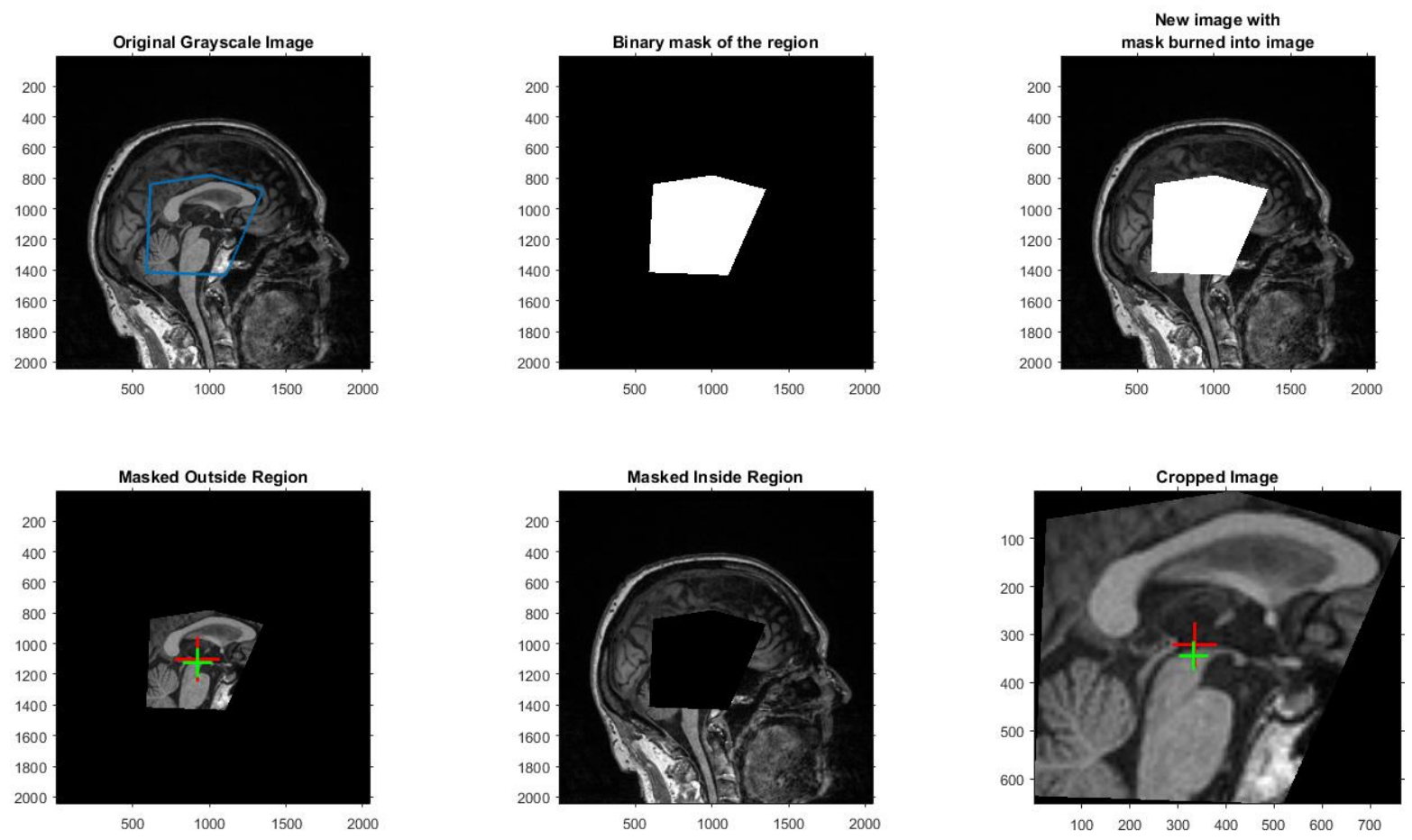

Figure 5.1: Sagittal ROI Process Extraction. 
The baseline image (top left) was marked with a polygon to select the ROI (hippocampus area). Then a binary mask was created to process the unmarked image (top center, and top left). The area and the centroid were applied to the baseline image, and then the masked was used to extract the region (center bottom image). The resulting image was the ROI with the centroid displayed at its center (bottom left)

\subsubsection{Axial images}

In addition to hippocampal atrophy, ventricular enlargement and cerebral cortex shrinkage have been associated with Alzheimer's disease [46] . Therefore, two different sections of the axial images were extracted: the ventricles, and the cerebral cortex portion. Consequently, two masks were created following the same process of convolution used for sagittal images. The axial image was the view from the NC baseline which was used to extract the sagittal view for analysis. Once a desire ventricle area was identified, the mask was created and utilized for all following images, (Figure 5.2). The cerebral cortex ROI was selected from the top right area of the axial images, and it was label thickness, (Figure 5.3). Two functions were created: one for ventricle extraction and one for cerebral cortex extraction. 

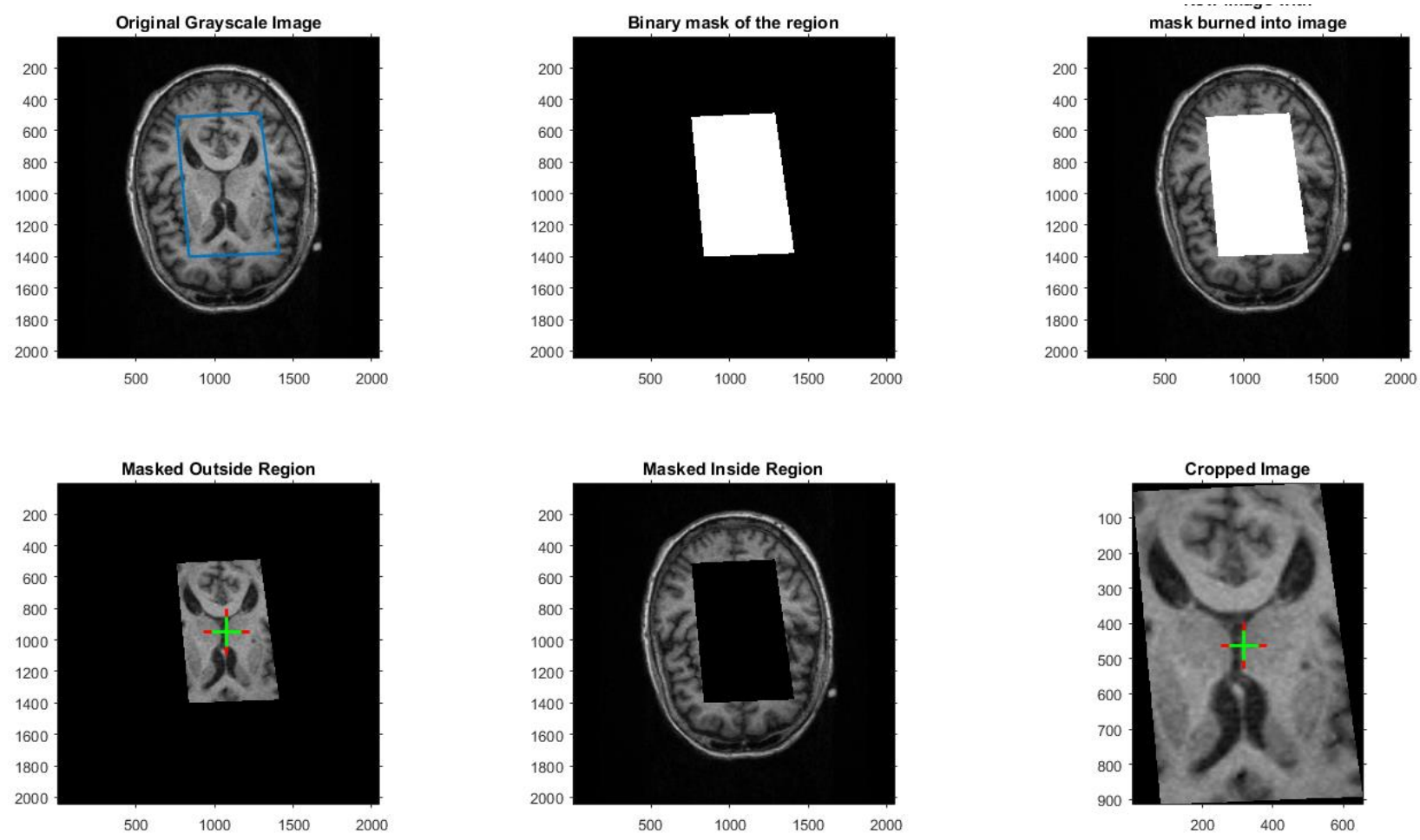

Figure 5.2: Axial ROI Process Extraction.

The baseline image (top left) was marked with a polygon to select the ROI (ventricle section). Then a binary mask was created to process the baseline image (top center, and top left). The area and centroid were applied to the baseline image, and the masked was used (center bottom image). The resulting image was the ROI with the centroid displayed at its center (bottom left) 

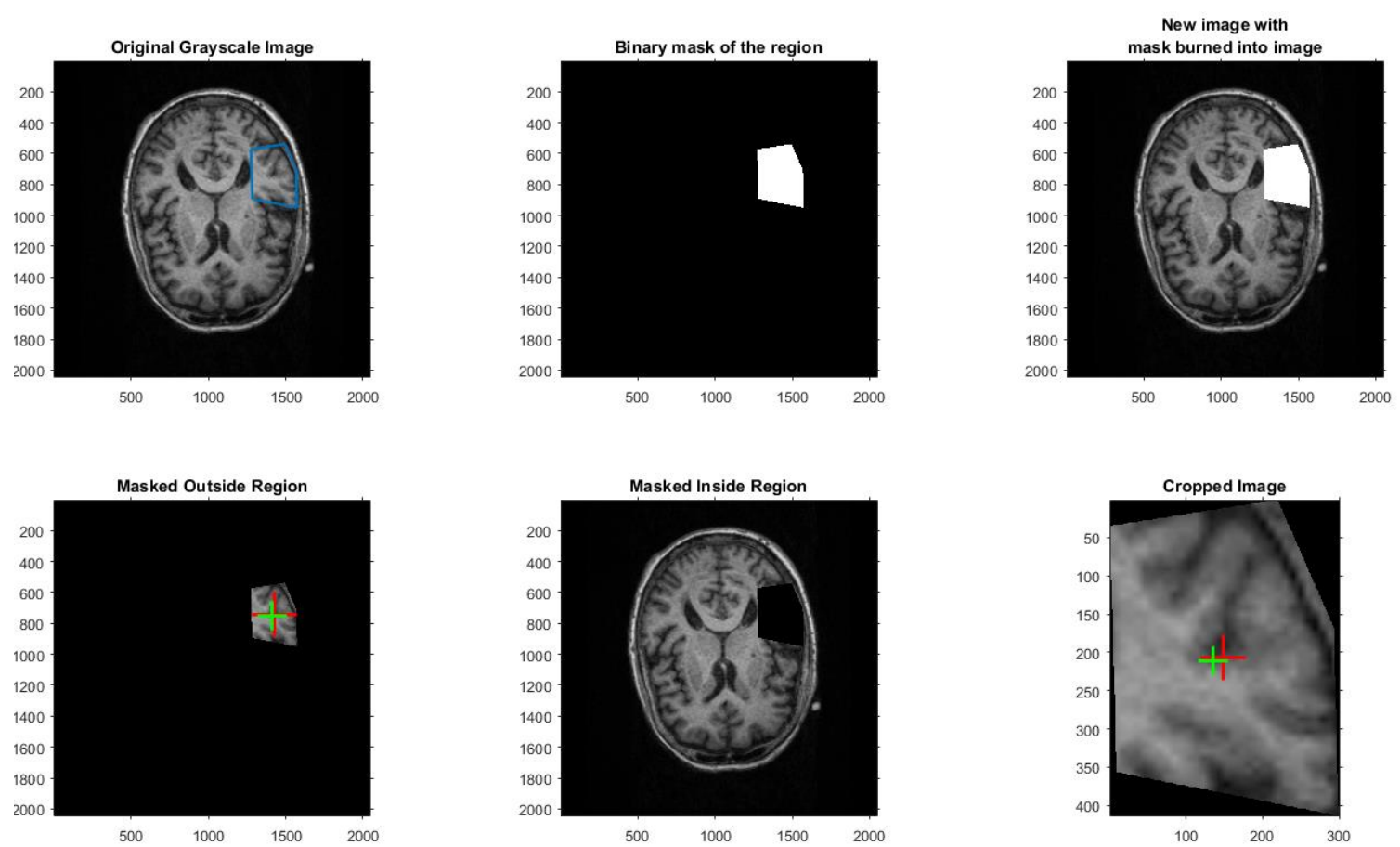

Figure 5.3: Thickness ROI Process Extraction.

The baseline image (top left) was marked with a polygon to select the ROI (Cortex thickness section). Then a binary mask was created to process the baseline image (top center, and top left). The area and centroid were applied to the baseline image, and the masked was used (center bottom image). The resulting image was the ROI with the centroid displayed at its center (bottom left)

\subsection{Image processing}

Mathematical morphology is a tool for extracting descriptive components of a picture which contains significant information. The binary mask used to remove the ROIs is an example of a mathematical morphology [39]. However, because of some of the morphological changes in brain deterioration, due to $\mathrm{AD}$, causes changes in volume, another image analysis comes into play. Texture analysis does not have a formal definition but refers to the descriptors which are statistical, structural, and spectral. The 
focus of this study is to use images as inputs, but retrieve attributes as outputs to use for classification.

\subsubsection{Frequency Domain Processing}

\subsubsection{Fourier Transform}

The Fourier transform gives properties primarily to detect global periodicity of an image. For a given image the Fourier transform extracts the magnitude and the phase, (Equation 5.2).

$$
F(u, v)=\sum_{x=0}^{M-1} \sum_{y=0}^{N-1} f(x, y) e^{-j 2 \pi u x / M} e^{-j 2 \pi u y / N}
$$

Where $\mathrm{F}(\mathrm{u}, \mathrm{v})$ is the discrete Fourier transform. $\mathrm{u}=0,1 ., \mathrm{M}-1$, and $\mathrm{v}=0,1 \ldots, \mathrm{N}-1$ and the result is a complex value. $\mathrm{f}(\mathrm{x}, \mathrm{y})$ is the given image, where $\mathrm{x}=0,1, \mathrm{M}-1$, and $\mathrm{y}=0,1, \mathrm{~N}-1$ [39].

All the images were converted to their Discrete Fourier Transform (DFT) which means that each picture were changed from their spatial domain to its frequency domain. In the frequency domain, the image is a series of sine and cosines. From this step, the magnitude of each MRI section was obtained, (Equation 5.3).

$$
f_{m a g}[m, n] \leftrightarrow|F[i, k]|
$$

Where the $f_{\text {mag }}[m, n]$ is the two-dimensional magnitude and $|F[i, k]|$ is the phase [39].

Both the magnitude and phase are complex values with real and imaginary parts.

All images were first converted to two-dimensional images by converting them from three color spectrums to two. 
The magnitude of the Fourier was calculated per equation $\mathbf{5 . 4}$

$$
f[m, n]=\sqrt{R(f(u, v))^{2}+i(f(u, v))^{2}}
$$

Where $f_{\text {mag }}[m, n]$ is the magnitude, $\mathrm{f}(\mathrm{u}, \mathrm{v})$ is the DTF, $\mathrm{R}$ is the real part and $\mathrm{i}$, is the imaginary part of the transform [39]. The magnitude provides information regarding the amount of frequency components present; therefore, the maximum and minimum values of the magnitude were stored as attributes.

The phase provides information regarding the location of the frequency components from which the real part is the focus of analysis. All images phase were calculated per equation $\mathbf{5 . 5}$

$$
|F[i, k]|=\tan ^{-1}\left(\frac{i(f(u, v)}{R(f(u, v)}\right)
$$

Where $F[i, k]$ is the magnitude, $i$ is the imaginary part of DTF and $R$ is the real part of the transform [39]. The maximum and minimum phase values of the real parts were part of the image analysis.

\subsubsection{Statistical approach}

The statistical approach can be used as a confirmation of the neural network performance since the data can be study to determine if there is a statistical significant difference among the parameters study. The statistical approach is not directly related to the aims of this work; however is a tool to study the data (inputs) being feat to the neural network.

\subsubsection{Properties of gray-level co-occurrence matrix}

The intensity of a picture can be obtained by a histogram which provides information about the intensity range of intensity levels in the image; however, the histogram 
provides not useful information for texture analysis. On the other hand, the analysis of image intensity with the relative position of the pixels provides valuable information. This combination is known as the gray level co-occurrence matrix (GLMC). A calculation of how often a gray-level pixel occurs in the horizontal adjacent direction of a pixel with a specific value is needed to obtain the matrix.

Q is the position of two pixels relative to each other, and the total number of pixel pairs that satisfies $Q$ is the sum of the elements of the matrix G. G contains the number of occurrences of pixels in image f. Then the probability that certain pair of points can satisfy Q is given by equation $\mathbf{5 . 6}$

$$
p_{i j}=g_{i j} / n
$$

Where $p_{i j}$ is the probability, $g_{i j}$ the pair of points, and $n$ is the total umber of pixel pairs that satisfy Q [39].

Thus, sum of probabilities equals one as they are in the range of $[0,1]$

$$
\sum_{i=1}^{k} \sum_{j=1}^{k} p_{i j}=1
$$

Where $\mathrm{k}$ is the dimension of the column or row of the square co-occurrence matrix $(\mathrm{G})$ [39].

Thus, all the images were converted to grayscale images, and the co-occurrence matrix was calculated for each image where the maximum probability of each image was stored for analysis. 


\subsubsection{Contrast}

The measure of the contrast is linked to the randomness of the content of an image. The value of contrast is the measure of intensity contrast between a pixel and its neighbor over the entire picture. (Equation 5.8)

$$
\text { Contrast }=\left[0\left(\sum_{i=1}^{k} \sum_{j=1}^{k}(i-j)^{2} P_{i j}\right)\right.
$$

When $\mathrm{G}$ (matrix) is constant, then the value of the range (contrast) is zero to $\mathrm{k}$ ( $\mathrm{k}$ is the row or column of the squared matrix $G$ [39]. Thus, contrast is the variance which measures the randomness with respect to the mean or how spread-out the pixels are from their GMLC values. The contrast of all three sets of images were obtained from the GLMC and stored as a texture attribute

\subsubsection{Correlation}

Correlation is a descriptor of the strength and direction of the relationship between the pixels and its neighbor over the entire images, which is a measure of redundancy. Correlation is calculated in the frequency domain and is a multiplication of complex conjugate values, (Equation 5.9).

$$
\begin{gathered}
\sum_{i=1}^{k} \sum_{j=1}^{k} \frac{\left(i-m_{r}\right)\left(j-m_{c}\right) p_{i j}}{\sigma_{r} \sigma_{c}} \\
\sigma_{r} \neq 0 ; \sigma_{c} \neq 0
\end{gathered}
$$

Where $m_{\mathrm{r}}$ is, the mean computed along the rows and $m_{\mathrm{c}}$ is the mean computed along the columns, $\sigma_{r}$, and $\sigma_{c}$ represent the standard deviations along the rows and columns respectively. The range is from 1 to -1 corresponding to the perfect positive and perfect 
negative correlations [39]. The correlation values for all images were obtained and saved as a feature for analysis

\subsubsection{Energy}

Energy is a measurement of uniformity with a range from 0 to 1 . A value of 1 represents a consistent image. Energy also is a second-moment equation, (Equation 5.10)

$$
E=\sum_{i=1}^{k} \sum_{j=1}^{k} p_{i j}^{2}
$$

Energy (E) is proportional to the probability squared; thus, as the probability squared increases, the uniformity of the image increases [39]. All the images energy was used as a statistical texture measured.

\subsubsection{Homogeneity}

Homogeneity $(\mathrm{H})$ measures the concentration of values or the spatial closeness of the distribution of the elements in $\mathrm{G}$ with respect to the main diagonal, (Equation 5.11).

$$
H=\sum_{i=1}^{k} \sum_{i=1}^{k} \frac{p_{i j}}{1+|i-j|}
$$

The value of $1+|i-j|$ are the same as the values of $\mathrm{G}$, and they decrease as $\mathrm{i}=\mathrm{j}$ [39]. Therefore, the highest homogeneity is the matrix with the highest probability near the main diagonal. Thus, images with higher gray level content have a higher homogeneity value, 1 . The range of homogeneity is from 0 to 1 . The values for all images were used as an attribute 


\subsubsection{Entropy}

Entropy (S) is the statistical analysis of randomness of the elements in $G$ and can be calculated in three or two dimensions, (Equation 5.12).

$$
S=-\sum_{i=1}^{k} \sum_{j=1}^{k} p_{i j} \log _{2}\left(p_{i j}\right)
$$

The randomness in the co-occurrence matrices is zero when the probability in all elements in $\mathrm{G}$ are constant; therefore, its range is [0,1] [39]. All the entropies were calculated and saved for analysis

\subsection{Artificial Neural Network}

The algorithm used for the classification of the data was the backpropagation algorithm, which is known to handle significant amounts of data and has been widely used in artificial intelligence models. The neural network consists of a forward pass, which knows the desire outputs and compares the calculated outputs with those already known. A second element is the backward pass where the error from the desired and actual output is computed. The resultant error from the backward pass is used to alter the weights in the network to reduce the error (Figure 5.4). Forward and backward passes are repeated until the error is low enough and converges to state where the learning patterns are bounded [47].

\subsubsection{Neural Network structure}

The neural network receives the data (input), then trains and processes the data. The inputs and biases are the connections among the inputs and outputs. The weights and biases are then summed and added up by a transfer function into one or more outputs 
depending on the process. There were four different structures compared which differed in the number of hidden layers and neurons in each layer, (Figure 5.5).

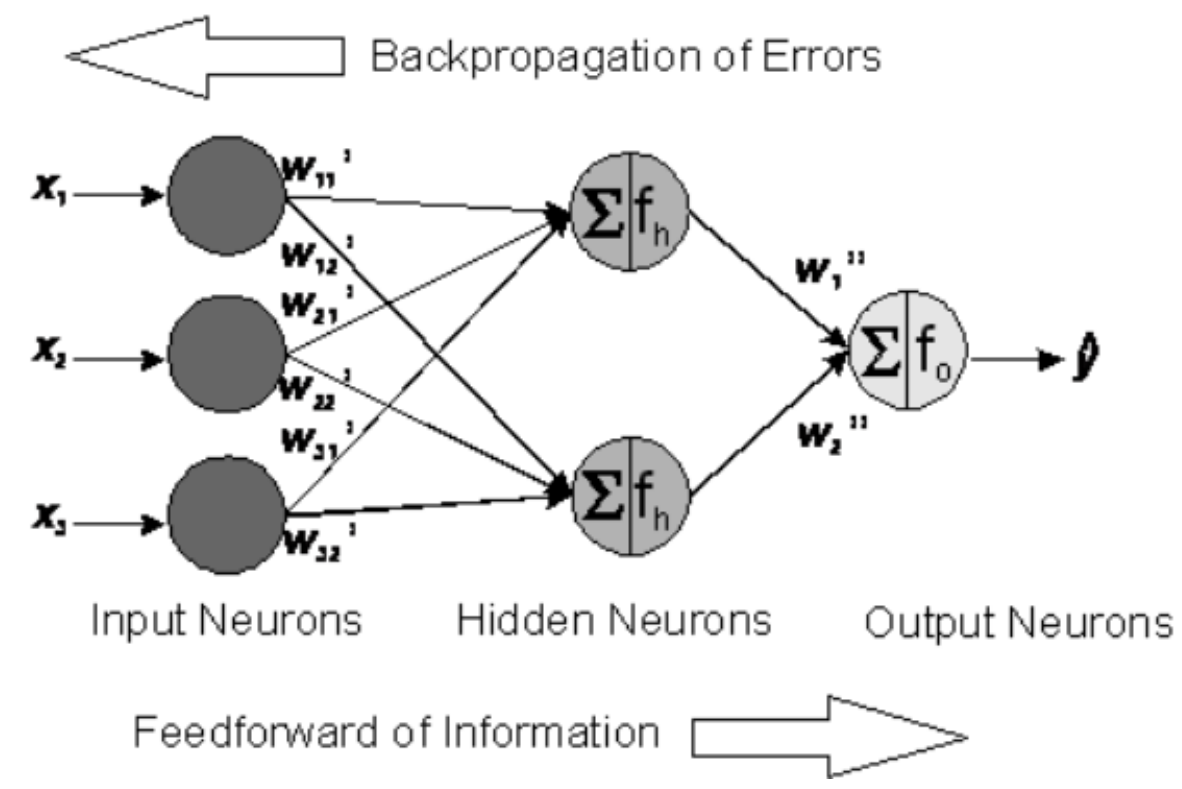

Figure 5.4: Multilayer Feedforward Backpropagation Artificial Neural Network

\section{Structure}

The neural network mimics the brain interconnecting structures and each neuron represent the information being received by the system. The neural network changes according to the structure selected for example this network shows three input variables $\left(\mathrm{x}_{1}, \mathrm{x}_{2}, \mathrm{x}_{3}\right)$ being received, but only one response variable $(\mathrm{y})$ being given. However, the number of inputs, hidden layers, and outputs can be increase or decrease as needed [48].

\subsubsection{Training Function and Cut off value}

The training function used was the trainlm, which uses the Levenberg-Marquardt optimization to update the weight and bias values. This methods was designed to approach the Hessian matrix rather than computing it (Equation 5.13, 5.14, 5.15)

$$
H=J^{T} J
$$




$$
g=J^{T} e
$$

Where $\mathrm{H}$ is the Hessian matrix, $\mathrm{g}$ is the gradient, and $\mathbf{J}$ is the Jacobian matrix with the first derivatives of the network errors in respect to the biases and weights, and e is a vector of networks. Therefore, the approximation of the Hessian matrix that the Levenberg-Marquardt employs following the Newton like update is shown by the following equation:

$$
X_{k+1}=X_{k}-\left[J^{T} J+\mu I\right]^{-1} J^{T} e
$$

$\mu$ is a scalar and when it equals zero the equation is Newton's method; however when $\mu$ is large then it becomes a gradient descent with a small reduction in performance function.

During this stage the cut-off value was arbitrarily chosen to be 0.5 ; thus, any output less than the cut-off value is consider negative. Similarly, any value equal or greater than 0.5 is assumed positive.

\subsection{Stage One Results}

\subsubsection{Features relevance}

One of the aims of this thesis was to determine the relevant features for comparison. A statistical t-test was performed among the 28 features to compare for the difference between the $\mathrm{AD}$ group and the $\mathrm{NC}$ group.

\subsubsection{Fourier Transform}

\subsubsection{Magnitude results}

The Fourier transform magnitude and phase for axial, thickness, and sagittal extractions were analyzed. The analysis of the magnitudes shows that there is no 
difference in the magnitude among groups for any of the views of the images, (Table 5.1).

Table 5.1: Summary of P-Value for Maximum and Minimum Magnitudes

\begin{tabular}{|l|l|l|}
\hline Image View & Maximum & Minimum magnitude \\
& magnitude & p-value \\
\hline Axial & 0.364 & \\
\hline Sagittal & 0.135 & 0.462 \\
\hline Thickness & 0.130 & 0.135 \\
\hline
\end{tabular}

There is not a significant statistical difference between minimum and maximum magnitudes for AD and NC groups in any of the views of the images analyzed.

\subsubsection{Phase results}

The phases arising from the Fourier transform were also analyzed, and the results show that there was a significant difference among the AD and NC images. The p-values for all image views show a significant statistical difference. The minimum thickness phase does not demonstrate a significant difference, (Table 5.2). 


\section{Table 5.2: Summary of P-Values for Minimum and Maximum Phase}

\begin{tabular}{|l|l|l|}
\hline Image Description & $\begin{array}{l}\text { Maximum phase } \\
\text { p-value }\end{array}$ & $\begin{array}{l}\text { Minimum phase } \\
\text { p-value }\end{array}$ \\
\hline Axial & 0.001 & 0.035 \\
\hline Sagittal & 0.000 & 0.992 \\
\hline Thickness & 0.028 & 0.876 \\
\hline
\end{tabular}

The p-values indicate that there is a statistical difference among the AD and NC for the axial view of the images. The results suggest that there is a 95 percent confidence that the true value of the standard deviation is in the range of 17 and 63 for the maximum and 1.9 and 51 for the minimum. Additionally, the sagittal view p-values also indicate that there is a significant statistical difference in the maximum phase of AD and NC with a $\mathrm{p}$-value of .000; however, the $\mathrm{p}$-value for minimum phase values was greater than .05 indicating that there is not a significant difference among the groups. Similarly, the results for thickness show a p-value of 0.028 which is less than .05 , showing that there is a significant statistical difference among the groups. However, like with the sagittal view, the minimum value indicates that there is not a significant difference among the groups.

\subsubsection{Contrast results}

A t-test statistical analysis with a 95 percent critical interval was performed in the contrast values. The comparison of all contrast values among the images led to statistical differences, (Table 5.3). 
Table 5.3: Summary of P-Values and Confidence Interval for Contrast

\begin{tabular}{|l|l|l|}
\hline Image View & Contrast & 95\% Confidence Interval \\
& p-value & \\
\hline Axial & 0.005 & $-0.00883,-0.00162$ \\
\hline Sagittal & 0.248 & $-0.00951,0.00248$ \\
\hline Thickness & 0.001 & $-0.01764,-0.00472$ \\
\hline
\end{tabular}

The contrast $\mathrm{p}$-values for axial and thickness views show a statistical significance among the groups with p-values of 0.005 and 0.001 , respectively. The sagittal view pvalue is greater than .05 , and the interval includes zero showing not a statistical difference among the AD and NC groups.

\subsubsection{Correlation results}

The correlation values among the $\mathrm{AD}$ and $\mathrm{NC}$ groups were analyzed using a t-test with a 95 percent confidence interval. The sagittal and thickness correlation values show that there is a statistical difference among the AD and NC groups with a p-value of .001 and 0 correspondently, (Table 5.4). 
Table 5.4: Summary of Correlation P-Values Among AD and NC groups

\begin{tabular}{|l|l|l|}
\hline Image View & $\begin{array}{l}\text { Contrast } \\
\text { p-value }\end{array}$ & Interval \\
\hline Axial & 0.79 & $-0.01125,0.02619$ \\
\hline Sagittal & 0.001 & $0.0213,0.0791$ \\
\hline Thickness & 0.000 & $0.0507,0.1296$ \\
\hline
\end{tabular}

The axial image view resulted in a p-value of .079 , which is greater than .05 and the interval contains zero; therefore, there it is concluded that there is not a statistical difference among the groups.

\subsubsection{Energy results}

The energy results for all images views among the AD and NC groups were analyzed using a t-test with a 95 percent confidence interval, (Table 5.5).

Table 5.5: Summary of Energy P-Values for Both AD and NC groups

\begin{tabular}{|l|l|l|}
\hline Image View & $\begin{array}{l}\text { Contrast } \\
\text { p-value }\end{array}$ & Interval \\
\hline Axial & 0.005 & $0.000793,0.004345$ \\
\hline Sagittal & 0.247 & $-0.00122,0.00468$ \\
\hline Thickness & 0.001 & $0.00234,0.00860$ \\
\hline
\end{tabular}


The energy analysis shows that there is a significant statistical difference among $\mathrm{AD}$ and NC groups for the axial and thickness views; however, the sagittal view resulted in a p-value of 0.247 . The large sagittal p-value shows that there is not a significant statistical difference among the groups which is confirmed by the presence of zero in its interval.

\subsubsection{Homogeneity}

The t-test with a 95 percent confidence interval was also calculated for all homogeneity measures. The homogeneity values show that the only significant statistical difference among the $\mathrm{AD}$ and $\mathrm{NC}$ groups is in the homogeneity of the sagittal view, (Table 5.6).

\section{Table 5.6: Summary of Homogeneity P-Values for AD and NC Groups}

\begin{tabular}{|l|l|l|}
\hline Image View & $\begin{array}{l}\text { Contrast } \\
\text { p-value }\end{array}$ & Confidence \\
& 0.310 & $-0.000674,0.002114$ \\
\hline Axial & 0.000 & $-0.005601,-0.001852$ \\
\hline Sagittal & 0.803 & $-0.00247,0.00191$ \\
\hline
\end{tabular}

The axial and thickness views p-values are higher than 0.05 which show no significant difference between the groups and it is confirmed the presence of zero among their intervals. 


\subsubsection{Entropy}

The statistical analysis of entropy among the AD and $\mathrm{NC}$ resulted in p-values for all axial, sagittal, and thickness views are below 0.05 . The low p-values show that there is a significant statistical difference among the groups, (Table 5.7).

Table 5.7: Summary of Entropy P-Values for All AD and NC Groups

\begin{tabular}{|c|c|c|}
\hline Image View & $\begin{array}{c}\text { Contrast } \\
\text { p-value }\end{array}$ & $\begin{array}{c}\text { 95\% Confidence } \\
\text { Interval }\end{array}$ \\
\hline Axial & 0.001 & $-0.1483,-0.0368$ \\
\hline Sagittal & 0.001 & $-0.2087,-0.0533$ \\
\hline Thickness & 0.000 & $-0.3126,-0.1160$ \\
\hline
\end{tabular}

Entropy p-values were consistent with all the views of the images. None of the ranges contain zero; therefore, it confirms that there is a significant statistical difference among the subjects.

\subsection{Artificial Neural Network}

\subsubsection{Classifier and performance evaluation}

The experimental results were analyzed with respect to four different percentages: accuracy, precision, sensitivity, and specificity. These allow for deeper insight and analysis of the classification results obtained. Sensitivity is a measure of how many true positives are correctly identified as true positive. Specificity is a measure of the number of true negatives which are correctly identified as being true negative [49]. Also, 
accuracy is a measure of how many of the predictions are correct, whereas precision is a measure of accuracy given that a certain class has been predicted [50].

For this study, a value of 1 is considered a negative since there is not AD associated with the image. A value of 2 is considered positive since the images are associated with the presence of AD. Then, a true positive is defined as a correctly classified AD presence, whereas a false positive is defined as an incorrectly classified AD presence. Furthermore, a true negative is defined as a correctly classified normal image with not AD present. A false negative is defined as an incorrect classification of a normal control. The degree at which the result of the algorithm conforms to the correct value provided by the dataset is referred to as accuracy (Equation 5.13):

$$
\text { Accuracy }=\frac{T P+T N}{T P+T N+F P+F N}
$$

Precision is defined as how exact and correct the values are when compared to the data (Equation 5.14)

$$
\text { Precision }=\frac{T P}{T P+F P}
$$

Additionally, sensitivity is the measurement of the true positive rate or the percentage of correctly identified true positives (Equation 5.15).

$$
\text { Sensitivity }=\frac{T P}{T P+F N}
$$

Finally, specificity refers to the true negative rate and correctly identifies the correct percentage of individuals not having the condition (Equation 5.16). 


$$
\text { Specificity }=\frac{T N}{T N+F P}
$$

TP represents the number of classified true positives, TN represents the number of classified true negatives, FP represents the number of falsely classified positives, and FN represents the number of incorrectly classified negatives.

The backpropagation ANN was trained with 50 percent of the data resulting in 124 samples. The testing and validation were done with the remaining 50 percent of the data points. There were four neural network structures analyzed to determine the best structure, (Table 5.8)

Table 5.8: ANN-Backpropagation Structure Results

\begin{tabular}{|l|l|l|l|l|}
\hline $\begin{array}{l}\text { Algorithm } \\
\text { structure }\end{array}$ & Accuracy & Sensitivity & Specificity \\
\hline $\begin{array}{l}\text { Network 1 } \\
2 \text { layers } \\
65 \text { neurons }\end{array}$ & 52.38 & 53.3 & 15.68 & 87.1 \\
\hline $\begin{array}{l}\text { Network 2 } \\
6 \text { layers } \\
65 \text { neurons }\end{array}$ & 53.3 & 56.3 & 17.7 & 87.04 \\
\hline $\begin{array}{l}\text { Network 3 } \\
4 \text { layers } \\
6 \text { neurons }\end{array}$ & 57.7 & 60 & 30 & 81.3 \\
\hline $\begin{array}{l}\text { Network 4 } \\
4 \text { layers } \\
20 \text { neurons }\end{array}$ & 53.3 & 55.0 & 21.6 & 83.3 \\
\hline
\end{tabular}


The network with 4 hidden layers and 35 neurons had the best performance with 91.3 percent specificity and 57.7 percent accuracy. The accuracy of all networks was within the 50 percent. The highest precision was 60 , and the lowest was 53.3 , achieved by network 3, and 1 respectively. The sensitivity value was very low for all four networks tested. The highest achieved value was from Network 4 with a high of 30 percent, while the worst was network 1 with a 15.7 percent. The networks with 65 neurons took a longer time and a higher computer power, while the networks with fewer neurons ran much faster and did not require a high-performance power. The number of epochs that resulted in the best performance ranged as low as 2 and as high as 8, (Figure 5.5). 


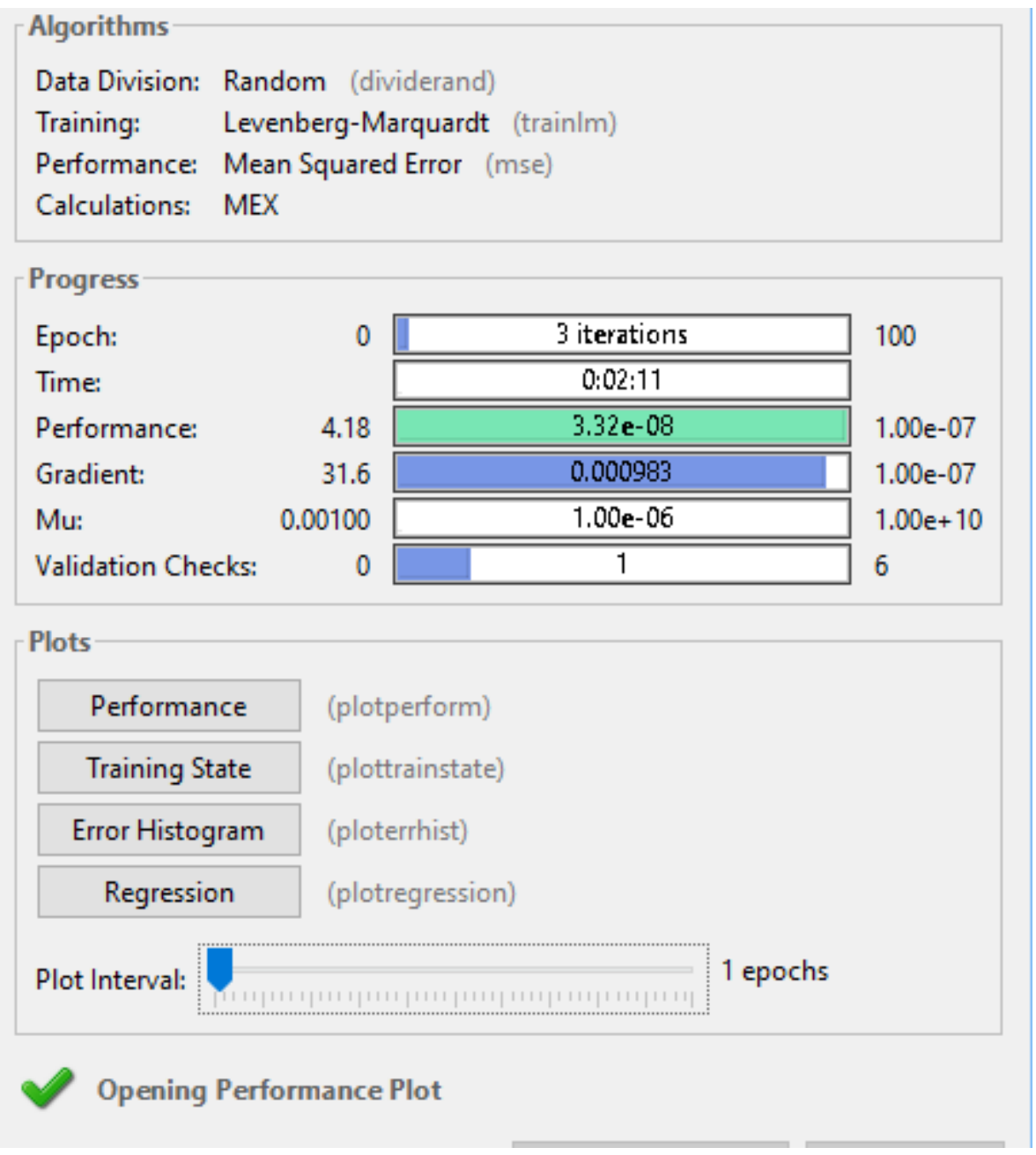

Figure 5.5: MATLAB Neural Network Interface.

Interface displays the type of training use, the epochs, time it took to run the simulation, and performance. From this interface plots for permeance, training, and error histogram were obtained. Furthermore, the interface shows the training function used, which was Levenberg-Marquardt [42].

An epoch refers the iteration where the validation performance reached a minimum, and training continued for .18 more runs. The same trend is seen for both the validation and test curve; therefore, there is no issue of overfitting, (Figure 5.6). 


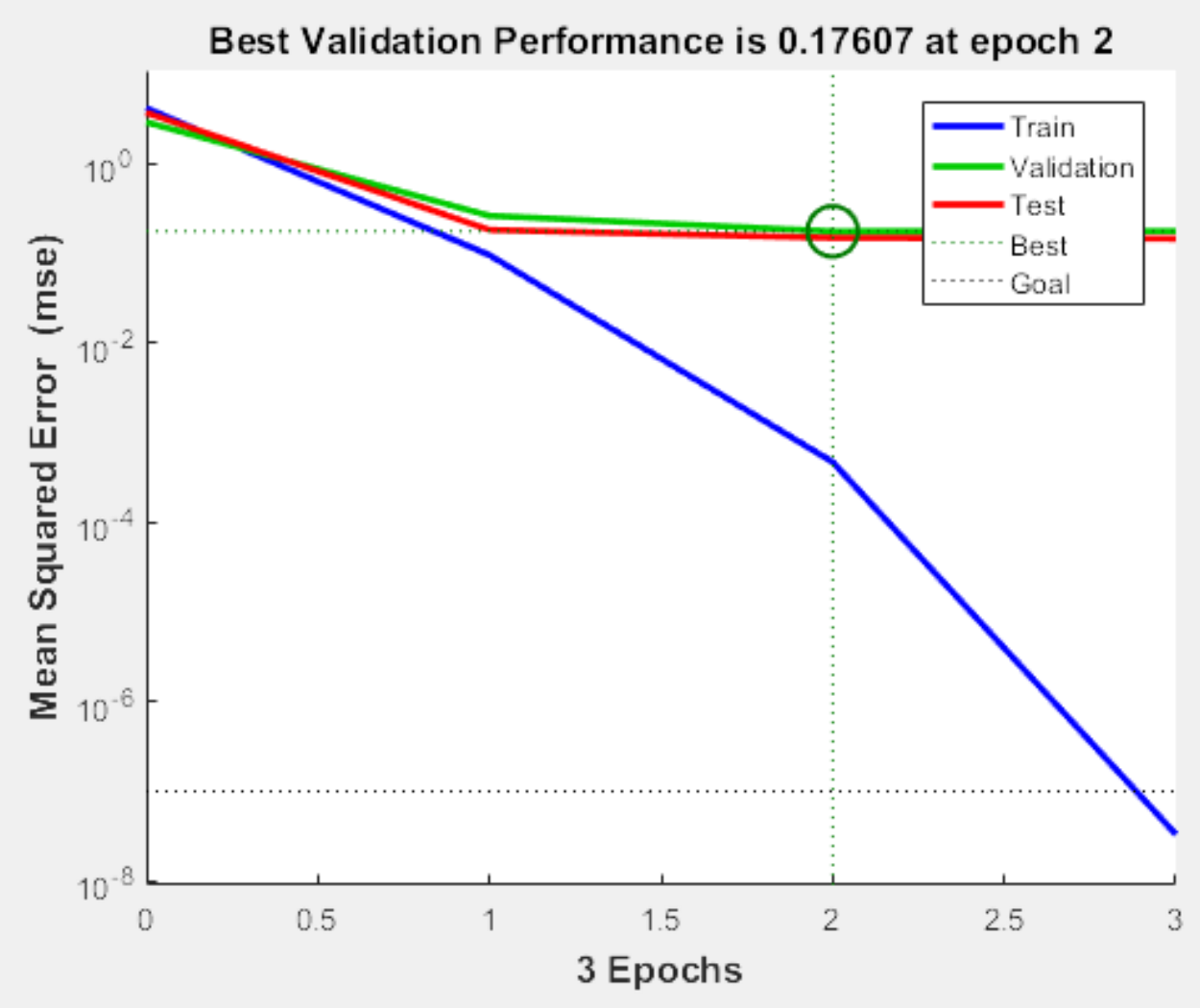

Figure 5.6: Performance Plot for Selected Network.

The network needed only an additional run to meet the goal and optimal point. The training curve (blue line) indicates that the information with the data might be limited; however, the validation (green line) and testing (red line) follow the same shape. The similarity among the curves shows that there is no problem of overfitting or overtraining; therefore, the performance of the network is generalizing.

The histogram of the data indicates that there are several out layers. The blue bars represent training data, the green bars represent validation data, and the red bars represent testing. The major out layers are found in validation and testing data 


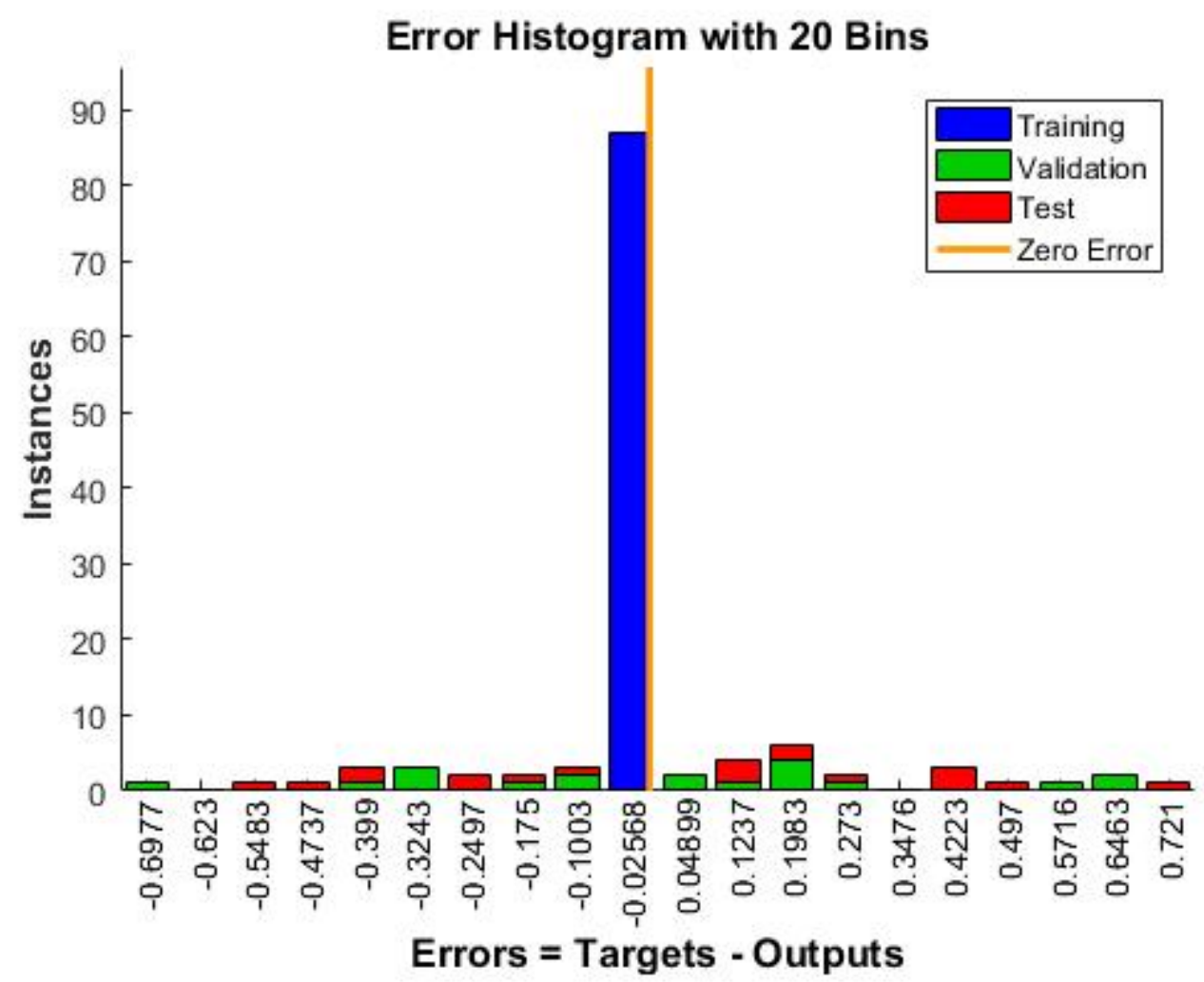

Figure 5.7: Histogram of Networks Performance.

The neural networks highest error rate happened during training in a 20-bit histogram. The training error can be the result of low data points.

The regression plots show that the targets and outputs are very close to each other. For training the R-value is 1 , so is for validation and testing. However, the data set is skewed and does not follow a normal distribution. 

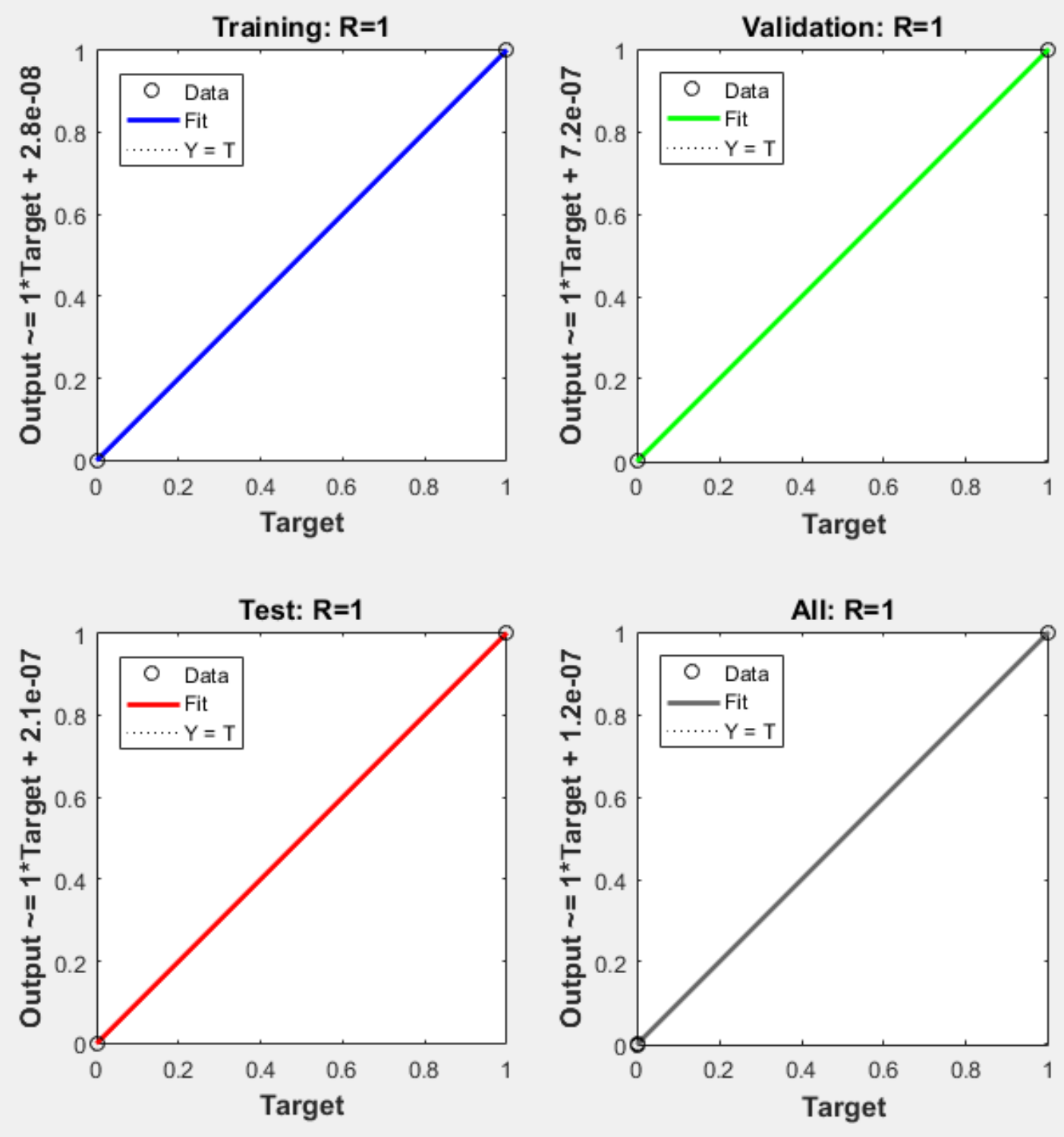

Figure 5.8: Regression Plot at Minimum Gradient of 15 epochs 


\subsection{Summary of Results}

The feature extraction results were summarized according to the extraction order. The results show that the maximum phase shift feature was statistically significant among the groups for all three views with p-values below .05, (Table 5.2). The minimum phase was only statistically significant for the axial view with a p-value of 0.035 . Contrast had a statistically significant difference in the axial and thickness views with p-values below .05, (Table 5.3). Similar results were observed for correlation where sagittal and thickness views were statistical significant with p-values below 0.05 ; while, the axial view showed no significant statistical difference, (Table 5.4). In the same way, energy was statistically significant for the thickness views, but in contrast the axial view was also statistical significant, (Table 5.5). Homogeneity was only statistically significant for the sagittal view, (Table 5.6), but energy p-values for all three views show that there is significant evidence to state a statistical difference among the groups.

The tabulated results from the algorithm performance correspond to the algorithms programmed with MATLAB using the 206 data points collected from all images giving a total number of 209 inputs, (Table 5.8). The best performance structure was the 4 layers 65 neural network with the highest specificity, but an underachieving accuracy, precision, and sensitivity to those compare with literature values. The literature values report all scores above the 90 percent, while the algorithm presented achieve values in the 50 percent for accuracy and precision [35]. The lowest performance value achieved was the sensitivity value with the highest score of 30 . 


\section{CHAPTER 6: STAGE TWO TEXTURE AND FREQUENCY RESULTS}

\subsection{Materials}

The number of subjects was increased to 300 images. There were 75 normal female controls, 75 normal male controls, 75 AD females, and 75 AD males. There was a significant effort to select similar age groups to keep a balance study; thus, the age range for all four groups was 55 to 85 years old. However, the average age for males and females in the normal control group was 73 years. The median age of AD patients, both male and females were 78 years of age.

\subsection{Methods}

The pilot study was used as a raw approach to refine the information being process from the MRI images. Thus, the purpose of stage two is to compare the frequency domain techniques, to compare the neural network structure, and to compare texture features against the frequency components. Moreover, to identify the method or methods with the highest performance that will give results comparable to those of the clinicians. During this phase, the only the axial view was used to compare the different techniques. The axial view was selected based on the statistical results from the pilot study. The results suggested that the axial view was the least useful to detect differences between the NC and AD groups. Thus, if the axial view performance is comparable to the clinician's, there is a higher probability that the other views have higher performance values than those of the clinicians. 


\subsection{Transforms comparison at low frequencies}

There are two main frequency approaches for image processing the Fourier transform (FT) and the discrete wavelet transform (DWT). Both techniques give different information; however, they can be compared in the low frequency domain. comparable using the low frequencies only.

\subsubsection{Fourier Transform (FT)}

The mathematical fundamentals of the FT were described earlier (5.4.1.1). During stage one, the maximum and the minimum values for the real parts of magnitude and phase shift were used for comparison. In contrast, for stage two the FT was obtained and then moved to the center. Additionally, a Gaussian low-pass filter was applied to eliminate the high frequencies, and the resulting transform was used for analysis. Due to the large volume of the resulting values due to the picture size, only a region was selected after processing. However, to evaluate if the area size impacted the results two different sizes were compared. In summary, a $3 \times 3$ window, which gives a total of 9 inputs, and a $4 \times 4$ window, which gave a total of 16 inputs, were compared.

\subsubsection{Discrete Wavelet Transform (DWT)}

The wavelet transforms as the Fourier transforms, is also in the frequency domain. The FT decomposes the signals into sine and cosines, and if the transform is taken over the entire axis, one particular frequency cannot be identified in a precise instant [39]. On the other hand, wavelet transforms are localized in both the real and Fourier space. Additionally, DWT returns a data vector which will have the same length as the input. The transform decomposes the data into a set of orthogonal wavelets many of the vector 
data are almost zero. Thus, there are some restrictions applied to the scaling function which is based on mathematical conditions per equation $6.1[38,48]$.

$$
f(x)=\sum_{k=-\infty}^{\infty} a_{k} \phi(S x-k)
$$

$\mathrm{S}$ is the scaling factor, $\mathrm{a}_{\mathrm{k}}$ is the set of coefficients, and $\phi(S x-k)$ is the expansion function. If the scaling function and wavelet function are given by any of the family such as Haar, Daubechies, and so on, then the bases are known. Therefore, the discrete signal in $1^{2}(Z)^{1}$ is per equation 6.2

$$
f[n]=\frac{1}{\sqrt{M}} \sum_{k} W_{\phi}\left[j_{0}, k\right] \phi_{j 0}, k[n]+\frac{1}{\sqrt{M}} \sum_{j=j_{0}}^{\infty} \sum_{k} W_{\psi}[j, k] \psi_{j, k}[n]
$$

Where $f[n], \phi_{j 0}$, and $\psi_{j, k}$ are discrete functions from 0 to $\mathrm{m}-1$. Thus, since the sets are orthogonal then their inner product gives the approximation coefficients according to equation 6.3 [51]

$$
W_{\phi}\left[j_{0}, k\right]=\frac{1}{\sqrt{M}} \sum_{n} f[n] \phi_{j 0}, k[n]
$$

A single-level two-dimensional wavelet decomposition was performed in all gray scale axial images. The DWT provides four different values a coefficient matrix value (cA), and three detail coefficients matrices for horizontal, vertical, and diagonal components (cH, cV, and $\mathrm{cD}$, respectively) [39]. However, only the coefficient matrix was used as it contains the low-frequency elements of the image. Due to the high volume of values as with the Fourier transform only a region was also selected. The same approach of a $3 \times 3$ and $4 \times 4$ windows were obtained. 


\subsubsection{Feature extraction}

Another approach to image processing is feature extraction, which was applied in stage one. The same features were utilized for phase two: contrast, correlation, homogeneity, energy, and entropy. Each texture gives four outputs; therefore, their entire combination is 16 inputs plus one more for entropy. Each of the features was analyzed independently, and as a combination (GLCOM); there was a total of four combination groups at different levels. A total of 12 combinations were used, which included a comparison of the four features together and in combination with entropy.

\subsection{Artificial Neural Networks (ANN)}

The artificial neural network approach and how it works was introduced on stage one. Step two compares four different parameters to identify the highest performance structures.

\subsubsection{Network structure}

Four different structures were analyzed with the axial images. Structure one and two were used to compare if increasing the number of neurons positively impacted performance. Therefore, one layer 15 neurons (structure 1:15), and one layer 35 neurons (structure 1:35) were used for comparison. Similarly, to compare the impact of increasing the number of layers the one layer 35 neurons (structure 1:35) was compared to the two layers 35 neurons structure (structure 2:35).

\subsubsection{Classifier and performance evaluation}

Stage two also used accuracy, precision, sensitivity, and specificity to compare the classifier performance; the definitions and mathematical description are layouts in section 5.7.1. In contrast to stage one, stage two uses 1 to identify individuals with AD, 
and 0 for NC. Additionally, to compare the impact of increasing training percentage, which reduces testing, two different percentages were used. All the different structures were run with 60 percent training; therefore, a 20 percent testing, and a 70 percent training with a 15 percent for testing.

The texture feature used the highest performing algorithm, which results from the combination of all five features. There were a total of four combination groups with sublevels: Combinations A with four sublevels, Combination B with three sublevels, Combination $\mathrm{C}$ with two sublevels and combination $\mathrm{D}$ with one sublevel. Additionally, each feature was tested for performance.

\subsubsection{Cutoff values}

The output of the classifier is in the range of 0 to 1 ; therefore, to be able to study the performance of the classifier a cutoff value is to be assigned. The cutoff value will determine what values correspond to 1 (AD group) or 0 (Normal control). In the case of training, the performance was evaluated using the value 0.5333 after moving up and down of 0.5 the 0.5333 gave the highest performance. After training the performance of testing was evaluated using the receiver operating characteristic (ROC) curve. The ROC curve finds the threshold or evaluates the performance in the regions of high sensitivity and high specificity. The function perfcurve is used to produce the ROC; there are not requirements or assumptions about the nature of inputs or their relationship. The perfcurve can be used with any classifier that returns scores ( 0 and 1$)$, which will return a point because the classification can be divided into positive or negative instances only. The test targets and the test output are used to find the threshold for the rate of positives and the rate of negatives after training [52]. The resulting value is used to evaluate the 
matrix with the true positives, true negatives, false positives, and false negative, which are used for accuracy, precision, sensitivity, and specificity.

\subsection{Stage two results}

The frequency transforms and texture features were compared based on their performance, which was evaluated with the four parameters: accuracy, precision, sensitivity, and specificity. The highest performing transforms were those that give comparable values to clinician's performance (71-87 percent sensitivity, and 44-71 percent specificity) simultaneously. Additionally, the performances that had values for sensitivity and specificity in a close range, and their accuracy approached 78 percent were evaluated as the highest performing algorithms. The performances which simultaneously gave values below the clinicians were assigned to low performance. Each structure was run five times, and the values for all runs were evaluated; however, only the maximum and minimum performances were tabulated for the discussion.

\subsubsection{Transform results and comparison}

The Fourier Transform and the Discrete Wavelet Transform were compared to determine, which of them provided enough and sensitive information for classification. Both transforms used a $3 \times 3$ window and a $4 \times 4$ window and only the low frequencies of the transforms were used. Using only the low frequencies allows for an even comparison of the performance of the transforms.

\subsubsection{Discrete Wavelet Transform (DWT) Results}

The DWT 3x3-window resulted in a nine input to the network, which was tested with the four structures. In the case of training, the highest performance was achieved with the 1:35 structure and a 60 percent training, while the lowest performing training 
was seen with structure 2:35 at 70 percent. In contrast, the lowest performance for testing was with the 60 percent training and structure 1:35, while the highest testing performance was achieved with 70 percent training and structure $1: 15$. For the $4 \times 4$ window and 16 inputs, the highest training performance was achieved with structure 1:15 and 60 percent training. Similarly, the lowest training, performance occurred also with the 60 percent training, but with structure 1:35. In the instance of testing the highest performing classification occurred with 70 percent training and structure 2:35, while the lowest performance was 60 percent training and structure 1:15,(Table 6.1, Table 6.2, Appendix A).

Table 6.1: Discrete Wavelet Transform training results for both window size

\begin{tabular}{|c|c|c|c|c|c|c|}
\hline \multirow{2}{*}{$\begin{array}{l}\text { Window } \\
\text { size }\end{array}$} & \multirow{2}{*}{ Structure } & \multicolumn{4}{|c|}{ Highest performing training results } & \multirow{2}{*}{$\begin{array}{l}\text { Percentage } \\
\text { for training }\end{array}$} \\
\hline & & Accuracy & Precision & Sensitivity & Specificity & \\
\hline$[3 \times 3]$ & $1: 35$ & 81 & 79 & 85 & 76 & 60 \\
\hline \multirow[t]{3}{*}[4\times4]{} & $2: 35$ & 91 & 92 & 92 & 91 & 70 \\
\hline & & \multicolumn{4}{|c|}{ Lowest performing training results } & \\
\hline & & Accuracy & Precision & Sensitivity & Specificity & \\
\hline$[3 \times 3]$ & $2: 35$ & 75 & 78.2 & 66.1 & 82.2 & 70 \\
\hline$[4 \times 4]$ & $1: 35$ & 62 & 60 & 62 & 62 & 60 \\
\hline
\end{tabular}


Table 6.2: Discrete Wavelet Transform testing results for both window sizes

\begin{tabular}{|c|c|c|c|c|c|c|}
\hline \multirow{2}{*}{$\begin{array}{l}\text { Window } \\
\text { size }\end{array}$} & \multirow{2}{*}{ Structure } & \multicolumn{4}{|c|}{ Highest performing testing results } & \multirow{2}{*}{$\begin{array}{l}\text { Percentage } \\
\text { for training }\end{array}$} \\
\hline & & Accuracy & Precision & Sensitivity & Specificity & \\
\hline$[3 \times 3]$ & $1: 15$ & 74 & 78 & 78 & 67 & 70 \\
\hline \multirow[t]{3}{*}[4\times4]{} & $2: 35$ & 78 & 80 & 50 & 93 & 70 \\
\hline & & \multicolumn{4}{|c|}{ Lowest performing testing results } & \\
\hline & & Accuracy & Precision & Sensitivity & Specificity & \\
\hline$[3 \times 3]$ & $1: 35$ & 63.3 & 60 & 64 & 63 & 60 \\
\hline$[4 \times 4]$ & $1: 15$ & 57 & 65 & 41 & 75 & 60 \\
\hline
\end{tabular}

\subsubsection{Fourier Transform (FT) Results}

The Fourier transform had significantly higher performances for all windows, percentages, and structures. In the case of the $3 \times 3$ window (9 inputs), the highest training performance occurred with structure 2:35 and 60 percent training, (Table 6.3, Appendix A). On the other hand, the lowest training performance was seen with 70 percent training and structure 2:35. Similarly, the highest testing performance occurred with 70 percent training and structure 1:15, while the lowest testing performance took place at structure 2:35 with 60 percent training, (Table 6.4). In the case of the 16 inputs $(4 \times 4)$, the highest performance training was 100 percent with structure 2:35 and 60 percent training, (Table 6.3, Appendix A). The lowest training performance occurred with 70 percent training and structure 1:15, (Table 6.3). The highest performance for testing occurred with 70 percent training and structure 2:35, while the lowest testing performance was structure 1:35with 60 percent training, (Table 6.4). 
Table 6.3: Fourier transforms training results for both window sizes.

\begin{tabular}{|c|c|c|c|c|c|c|}
\hline \multirow{2}{*}{$\begin{array}{l}\text { Window } \\
\text { size }\end{array}$} & \multirow{2}{*}{ Structure } & \multicolumn{4}{|c|}{ Highest performing training results } & \multirow{2}{*}{$\begin{array}{l}\text { Percentage } \\
\text { for training }\end{array}$} \\
\hline & & Accuracy & Precision & Sensitivity & Specificity & \\
\hline$[3 \times 3]$ & $1: 15$ & 86 & 85 & 87 & 84 & 60 \\
\hline \multirow[t]{3}{*}[4\times4]{} & $2: 35$ & 100 & 100 & 100 & 100 & 60 \\
\hline & & \multicolumn{4}{|c|}{ Lowest performing training results } & \\
\hline & & Accuracy & Precision & Sensitivity & Specificity & \\
\hline$[3 \times 3]$ & 2:35 & 69 & 67 & 84 & 53 & 70 \\
\hline$[4 \times 4]$ & $1: 15$ & 68 & 85 & 74 & 91 & 60 \\
\hline
\end{tabular}

Table 6.4: Fourier Transform testing results for both widow sizes.

\begin{tabular}{|c|c|c|c|c|c|c|}
\hline \multirow{2}{*}{$\begin{array}{l}\text { Window } \\
\text { size }\end{array}$} & \multirow{2}{*}{ Structure } & \multicolumn{4}{|c|}{ Highest performing testing results } & \multirow{2}{*}{$\begin{array}{l}\text { Percentage } \\
\text { for training }\end{array}$} \\
\hline & & Accuracy & Precision & Sensitivity & Specificity & \\
\hline$[3 \times 3]$ & $1: 15$ & 85 & 87 & 90 & 75 & 70 \\
\hline \multirow[t]{3}{*}[4\times4]{} & $1: 35$ & 82 & 83 & 90 & 71 & 70 \\
\hline & & \multicolumn{4}{|c|}{ Lowest performing testing results } & \\
\hline & & Accuracy & Precision & Sensitivity & Specificity & \\
\hline$[3 \times 3]$ & $1: 35$ & 73 & 67 & 68 & 50 & 60 \\
\hline$[4 \times 4]$ & $1: 15$ & 77 & 74 & 79 & 74 & 60 \\
\hline
\end{tabular}


The overall performance for training and testing suggest that the Fourier transform perform has higher sensitivity and specificity while compared to the DWT. The maximum performing transform was the FT with a $4 x 4$ window.

\subsubsection{Texture features (TF)}

The texture features (TF) performance was also evaluated. The texture features from the gray-level co-occurrence matrix (GLCOM) were evaluated as a single entity (contrast, correlation, energy, and homogeneity). Additionally, the GLCOM plus entropy was also evaluated. Furthermore, each TF was individually evaluated, as well as in a series of combinations at different levels. Thus, the first comparison is the single entity vs. single entity plus entropy. The second comparison will be the independent performance of each, and the third comparison will be the combination performance.

\subsubsection{Texture features and entropy results}

The combinations of the four features making the GLCOM and the GLCOM in combination with entropy were measured for performance during training and testing. (Table 6.5,Table 6.6).Table 6.5: Training results for Gray level co-occurrence matrix (GLCOM), and Gray level co-occurrence matrix (GLCOM) + entropy

\begin{tabular}{|c|c|c|c|c|}
\hline & \multicolumn{4}{|c|}{ Highest training performance } \\
\hline & Accuracy & Precision & Sensitivity & Specificity \\
\hline GLCOM & 70 & 72 & 67 & 72 \\
\hline \multirow[t]{2}{*}{$\begin{array}{l}\text { GLCOM + } \\
\text { entropy }\end{array}$} & 84 & 84 & 83 & 84 \\
\hline & \multicolumn{4}{|c|}{ Lowest training performance } \\
\hline GLCOM & 64 & 67 & 59 & 71 \\
\hline $\begin{array}{l}\text { GLCOM + } \\
\text { entropy }\end{array}$ & 61 & 59 & 64 & 54 \\
\hline
\end{tabular}


Table 6.6: Testing results for Gray level co-occurrence matrix (GLCOM), and Gray level co-occurrence matrix (GLCOM) + entropy

\begin{tabular}{|c|c|c|c|c|}
\hline \multirow{2}{*}{} & \multicolumn{4}{|c|}{ Highest testing performance } \\
\cline { 2 - 5 } & Accuracy & Precision & Sensitivity & Specificity \\
\hline GLCOM & 82 & 86 & 86 & 76 \\
\hline $\begin{array}{l}\text { GLCOM } \\
+ \\
\text { entropy }\end{array}$ & 80 & 90 & 71 & 91 \\
\hline & \multicolumn{2}{|c|}{ Lowest testing performance } & \\
\hline $\begin{array}{l}\text { GLCOM } \\
\text { GLCOM } \\
+\end{array}$ & 76 & 76 & 86 & 67 \\
entropy & 56 & 54 & 60 & 52 \\
\hline
\end{tabular}

The results suggest that entropy lowers the performance of the network during the testing stage; however, during training, the results suggest that entropy values are much higher. On the other hand, entropy significantly reduces the testing performance as its lowest values are about 20 points below the clinician's highest performance. Conversely, the testing without entropy gave values slightly below than the maximum performance of the clinicians. Moreover, the values were much higher than the minimum values recorded for sensitivity and specificity. Accuracy was still 2 points less than the reported for the clinicians.

\subsubsection{Individual texture features performance}

Each independent feature was its own output; therefore, a four input was used to test performance. Using the results from the transforms the individual features were only evaluated with structure 1:15 and 70 percent training. The highest and lowest performance for both training and testing were recorded. 
In the case of the individual features the training performance was lower than the clinicians for accuracy, and sensitivity; however, the values for specificity were higher than the maximum performance given by the clinicians by up to 20 points, (Table 6.7). In contrast, the testing performance gave comparable and slightly higher performance values than those of the clinician, (Table 6.8). The two most top performing features were contrast and correlation. Contrast had the same accuracy as the clinician and greater specificity; however, its sensitivity was only higher than the minimum value recorded from clinicians. Similarly, the correlation was higher in accuracy and specificity, but slightly lower than the maximum sensitivity of the clinicians by one point, (Table 6.7, Table 6.8).

Table 6.7: Training performance for individual texture features

\begin{tabular}{|l|c|c|c|c|c|c|c|c|}
\hline \multirow{2}{*}{ Features } & \multicolumn{4}{|c|}{ Highest training performance } & \multicolumn{3}{c|}{ Lowest training performance } \\
\cline { 2 - 10 } & Acc. & Prec. & Sens. & Spec. & Acc. & Prec. & Sens. & Spec. \\
\hline Contrast & 70 & 75 & 59 & 81 & 54 & 61 & 34 & 61 \\
\hline Correlation & 67 & 67 & 63 & 71 & 65 & 68 & 64 & 67 \\
\hline Energy & 62 & 63 & 75 & 47 & 54 & 58 & 57 & 59 \\
\hline Homogeneity & 66 & 70 & 71 & 60 & 57 & 58 & 57 & 59 \\
\hline Entropy & 62 & 67 & 42 & 81 & 57 & 52 & 22 & 92 \\
\hline
\end{tabular}


Table 6.8: Testing performance for individual texture features

\begin{tabular}{|l|c|c|c|c|c|c|c|c|}
\hline \multirow{2}{*}{ Features } & \multicolumn{4}{|c|}{ Highest testing performance } & \multicolumn{3}{c|}{ Lowest testing performance } \\
\cline { 2 - 9 } & Acc. & Prec. & Sens. & Spec. & Acc. & Prec. & Sens. & Spec. \\
\hline Contrast & 78 & 76 & 76 & 79 & 60 & 54 & 74 & 67 \\
\hline Correlation & 79 & 75 & 86 & 74 & 78 & 71 & 91 & 67 \\
\hline Energy & 76 & 78 & 85 & 61 & 65 & 61 & 67 & 62 \\
\hline Homogeneity & 74 & 72 & 84 & 60 & 60 & 67 & 62 & 58 \\
\hline Entropy & 76 & 70 & 74 & 77 & 54 & 57 & 68 & 44 \\
\hline
\end{tabular}

\subsubsection{Combination of features}

A series of possible combinations were created to test if one combination of features is preferable than another. Also, the combo features were only tested with structure 1:35 and training of 70 percent, (Table 6.9, Appendix B). There were a total of four combinations umbrellas with sub-combination numbers: Combo A (AI, AII, AIII, AIV), Combo B (II, III, IV), Combo C (III, IV), and combo D. Combination A is all combinations related to contrast, combination B compares correlation in conjunction with the other features excluding contrast, combo C compares energy against homogeneity and entropy, and combo D evaluates the combination of homogeneity and entropy. (Table 6.9, Table 6.10, Appendix C). 


\section{Table 6.9: Training results for combination $\mathrm{A}$}

\begin{tabular}{|l|c|c|c|c|c|c|c|c|}
\hline \multirow{2}{*}{ Combination } & \multicolumn{4}{|c|}{ Highest training performance } & \multicolumn{3}{c|}{ Lowest training performance } \\
\cline { 2 - 10 } & Acc. & Prec. & Sens. & Spec. & Acc. & Prec. & Sens. & Spec. \\
\hline AI & 79 & 83 & 74 & 84 & 66 & 64 & 62 & 68 \\
\hline All & 64 & 66 & 61 & 64 & 69 & 74 & 57 & 81 \\
\hline AIII & 78 & 73 & 80 & 76 & 71 & 71 & 74 & 69 \\
\hline AIV & 73 & 76 & 66 & 54 & 54 & 56 & 66 & 52 \\
\hline
\end{tabular}

AI and AIII had the highest training performance. Combination AI is contrast and correlation together, which gave an accuracy value greater than the clinician's as well as specificity. However, in the case of sensitivity, the values were slightly higher than the minimum reported for the clinicians. The training values were lower to those of the clinicians for all values. Combination AIII was contrast and homogeneity, and their training performance was higher for sensitivity while compared to combo AII, (Table 6.9). However, its performance was lower than the highest performance reported for the clinicians by 6 points. On the other hand, accuracy was comparable to the clinicians and specificity was greater than the maximum clinician's range. In the case of the lowest training performance combination, AIII had higher values to those for combination AI where both sensitivity and accuracy were greater than the minimum performance for the clinicians. (Table 6.10) 
Table 6.10: Testing results for combination $A$

\begin{tabular}{|l|c|c|c|c|c|c|c|c|}
\hline \multirow{2}{*}{ Combination } & \multicolumn{4}{|c|}{ Highest testing performance } & \multicolumn{3}{c|}{ Lowest testing performance } \\
\cline { 2 - 10 } & Acc. & Prec. & Sens. & Spec. & Acc. & Prec. & Sens. & Spec. \\
\hline Al & 78 & 75 & 88 & 67 & 60 & 59 & 48 & 71 \\
\hline All & 76 & 76 & 85 & 64 & 55 & 57 & 55 & 56 \\
\hline Alll & 71 & 74 & 67 & 76 & 56 & 54 & 66 & 46 \\
\hline AIV & 73 & 77 & 77 & 68 & 67 & 69 & 77 & 53 \\
\hline
\end{tabular}

In the case of testing the highest performance was AI and AII. AIII accuracy was lower than the values for the clinicians and AI and AII. In the case of Sensitivity, the values for AIII were significantly lower than for the other two combinations and then the lowest value reported for the clinicians. On the other hand, combination AII had higher values than AIII,

but lower than the clinician's highest performing values. Thus, combination AI outperforms the testing performance having equivalent values to clinician's accuracy, and outperforming in sensitivity. The specificity value was slightly lower than those for the clinicians.

The next set included three different combinations with correlation, (Appendix C). The highest training performing combinations for set B were BI and BII with specificity of 78 , which is much greater than the one reported for the clinicians. On the other hand, the accuracy was three points below than the clinicians and the sensitivity was comparable to that of the clinicians. Combination BIII gave slightly lower values for accuracy compared to the other two combinations, higher precision, comparable 
specificity, and much lower sensitivity than the one reported for the clinician's lower range, (Table 6.11)

Table 6.11: Training results for combination $B$

\begin{tabular}{|l|c|c|c|c|c|c|c|c|}
\hline \multirow{2}{*}{ Combination } & \multicolumn{4}{|c|}{ Highest training performance } & \multicolumn{3}{c|}{ Lowest training performance } \\
\cline { 2 - 10 } & Acc. & Prec. & Sens. & Spec. & Acc. & Prec. & Sens. & Spec. \\
\hline BI & 75 & 74 & 71 & 78 & 67 & 64 & 67 & 66 \\
\hline BII & 75 & 77 & 71 & 78 & 68 & 70 & 67 & 70 \\
\hline BIII & 74 & 80 & 63 & 71 & 57 & 68 & 24 & 84 \\
\hline
\end{tabular}

Contradictorily to the training results, the values for testing were much different for combination BIII. BIII was the combination of correlation and entropy which outperformed the clinician's value for accuracy by up to 5 points, and specificity by up to 19 points. On the other hand, sensitivity was lower than the highest reported value for clinician's performance, but much greater than the lower range for the clinicians, (Table 6.12).

Table 6.12: Testing results for combination B

\begin{tabular}{|l|c|c|c|c|c|c|c|c|}
\hline \multirow{2}{*}{ Combination } & \multicolumn{4}{|c|}{ Highest testing performance } & \multicolumn{3}{c|}{ Lowest testing performance } \\
\cline { 2 - 10 } & Acc. & Prec. & Sens. & Spec. & Acc. & Prec. & Sens. & Spec. \\
\hline BI & 78 & 81 & 74 & 82 & 67 & 67 & 50 & 80 \\
\hline BII & 73 & 74 & 90 & 47 & 56 & 54 & 64 & 48 \\
\hline BIII & 82 & 91 & 76 & 90 & 62 & 57 & 60 & 64 \\
\hline
\end{tabular}

Two combinations including energy composed set $\mathrm{C}$ and combination $\mathrm{D}$ was Homogeneity and entropy. In the case of training, the three combinations were underperforming on all four parameters. The highest combination CII (energy and 
entropy) had an accuracy of 73 , precision of 68 , sensitivity of 60 , and specificity of 61 which were much lower compared to the clinician's, (Table 6.13). 
Table 6.13: Training results for combination $\mathrm{C}$ and $\mathrm{D}$

\begin{tabular}{|l|c|c|c|c|c|c|c|c|}
\hline \multirow{2}{*}{ Combination } & \multicolumn{4}{|c|}{ Highest training performance } & \multicolumn{3}{c|}{ Lowest training performance } \\
\cline { 2 - 10 } & Acc. & Prec. & Sens. & Spec. & Acc. & Prec. & Sens. & Spec. \\
\hline $\mathrm{Cl}$ & 70 & 75 & 62 & 78 & 61 & 67 & 53 & 70 \\
\hline $\mathrm{Cll}$ & 73 & 68 & 60 & 61 & 57 & 66 & 40 & 75 \\
\hline $\mathrm{D}$ & 67 & 71 & 63 & 71 & 57 & 58 & 60 & 54 \\
\hline
\end{tabular}

In the case of testing combination CII had more promising values as accuracy was comparable to the clinicians, and sensitivity and specificity slightly higher than those maximum values reported from clinician's performance, (Table 6.14).

Table 6.14: Testing results for combination $C$ and $D$

\begin{tabular}{|l|c|c|c|c|c|c|c|c|}
\hline \multirow{2}{*}{ Combination } & \multicolumn{4}{|c|}{ Highest testing performance } & \multicolumn{3}{c|}{ Lowest testing performance } \\
\cline { 2 - 10 } & Acc. & Prec. & Sens. & Spec. & Acc. & Prec. & Sens. & Spec. \\
\hline Cl & 76 & 72 & 96 & 44 & 64 & 58 & 70 & 60 \\
\hline CII & 78 & 78 & 88 & 72 & 76 & 76 & 80 & 72 \\
\hline D & 77 & 71 & 60 & 80 & 64 & 63 & 78 & 50 \\
\hline
\end{tabular}

\subsection{Statistical results}

From the logistic regression model, the p-value for all texture features, Fourier transform, and Discrete wavelet transform was obtained. The statistical analysis shows that the TFs are statistically different between $\mathrm{AD}$ and $\mathrm{NC}$ 's as all their p-values were below .05 ,

\section{(Appendix D).}

The frequency transform had similar results were the statistical analysis shows that there was a significant statistical difference between $\mathrm{AD}$ and $\mathrm{NC}$ 's for both FT 
windows, and for the DWT $3 \times 3$ window only. However, the statistical p-value for the DWT with a window size of $4 \times 4$ was not statistically significant for $\mathrm{AD}$ and $\mathrm{NC}$ 's,

\section{(Appendix E).}

\subsection{Summary of Results}

The training for both FT and DWT was much higher for the $4 \times 4$ window than for the $3 \times 3$ region, which was also the same in the case of performance for the DWT, but not for the FT. The Fourier transform achieved training values up to 100 percent in all four parameters, and its higher training performance outperformed the DWT and the clinician's values. In contrast to DWT, the FT testing performance was similar for both windows size. The $3 \times 3$ window had slightly higher values than the $4 \times 4$ window, but the $4 \mathrm{x} 4$ window lowest performance was closer to the clinician's results. Overall, the highest performing algorithm on both training and testing was the FT with values outperforming the clinician's (accuracy of 85 , a precision of 87 , sensitivity of 90 , and specify of 75 ). In conclusion using a 70 percent training resulted in higher performance for testing, but lower for training. The fewer neuron structures (1:15) were favorable to instances were fewer inputs were used, and the increase of neurons favor a higher number of inputs (1:35). The addition of layers did not seem to improve performance.

The combination of the sixteen values for contrast, correlation, energy, and homogeneity were compared to those same four plus entropy. In the case of training, the highest performance was in combination with entropy having much higher values than the clinician's in accuracy and specificity and closer values to the maximum range for sensitivity. In contrast, the testing shows that the single entity (four features only) had much higher values for accuracy and specificity. Sensitivity was one-point lower than the 
clinician's performance and precision was 86 percent. The most inferior testing performance was also significantly better for a single entity than with the combination of entropy.

The individual features performance was evaluated, and the lowest training performance was seen by entropy alone. The highest training performing feature was that of contrast; however, none of the training gave comparable values to the clinicians. Moreover, the training performance was significantly lower than those of the clinicians. Nevertheless, the correlation's testing performance was higher than the clinicians in the case of accuracy and specificity; sensitivity was one-point lower than the most top performing range of the physician's 86 percent.

In the event of the combinations, the training and testing for combination $\mathrm{A}$ resulted in combination $\mathrm{AI}$ as the highest performing combination. AI training performance was greater than the clinician's performance in accuracy, outperformed in specificity, and was larger than the lower range in sensitivity. For testing AI outperformed the clinicians in both accuracy and sensitivity; however, it was slightly less than the upper range of the clinician's. None of the training performance of combination B was higher than combination $\mathrm{A}$, and none of the combinations outperform the clinicians in two simultaneous values. In the case of testing performance, BI was comparable to the clinicians in accuracy, slightly higher than the minimum value in sensitivity, and outperformed in specificity. On the other hand, BIII significantly outperformed in accuracy and specificity, and its sensitivity was higher than the lower range. A similar trend was seen for combination $\mathrm{C}$ where the training performance was below the clinician's values; however, the testing shows that CII outperformed the 
clinicians in sensitivity, and upper specificity ranges, and was comparable in accuracy. Thus, single entity slightly exceeded the clinicians and the combination with entropy. Moreover, the correlation was the highest performing texture feature outperforming the clinician's performances in two values simultaneously. Combination CII outperformed all other combinations in testing. Furthermore, it outperformed the clinicians in sensitivity and specificity and was comparable in accuracy. 


\section{CHAPTER 7: STAGE THREE VIEW COMPARISON RESULTS}

\subsection{Materials}

The same database and demographics used in stage two were applied to stage three. The fundamental difference of this phase was the addition of two views coronal and sagittal. There was a total of 300 images from coronal and 300 from sagittal used for the comparison.

\subsection{Methods}

The purpose of this stage was to determine if adding additional MRI views will increase performance, or one single view suffices for a comparable classification. Thus, the coronal and sagittal view were processed according to the results of stage two where the highest frequency techniques are being used; thus, the FT of each view. The GLCOM was also used to compare each view's performance, but as with the FT not in the combination of all views due to the volume of inputs. To examine if a mix of views leads to higher performance the greatest texture feature (correlation) and the most top combination of features (CII) will be used for the analysis.

\subsubsection{Fourier transforms}

The three views were compared for performance during training and testing. The comparison of the views aims to determine if one view is more favorable than the other. In the case of the FT the axial view outperformed the other two views during training performance; however, the other two views also outperformed the clinicians at accuracy and specificity and were comparable in sensitivity, (Table 7.1). 
Table 7.1: Training performance of each view's Fourier Transform

\begin{tabular}{|l|c|c|c|c|c|c|c|c|}
\hline \multirow{2}{*}{ View } & \multicolumn{4}{|c|}{ Highest training performance } & \multicolumn{3}{c|}{ Lowest training performance } \\
\cline { 2 - 10 } & Acc. & Prec. & Sens. & Spec. & Acc. & Prec. & Sens. & Spec. \\
\hline Axial & 86 & 81 & 94 & 77 & 71 & 67 & 77 & 65 \\
\hline Coronal & 81 & 83 & 78 & 82 & 62 & 63 & 53 & 61 \\
\hline Sagittal & 80 & 82 & 78 & 83 & 67 & 67 & 73 & 61 \\
\hline
\end{tabular}

In the case of testing the axial view had a higher performance than the other views. The second-best view was the coronal also outperforming the maximum performance of the clinicians. In contrast, the sagittal view best testing performance only compared to the clinician's accuracy. It outperformed the clinician's sensitivity but was only higher to the lowest range for specificity, (Table 7.2).

Table 7.2: Testing performance of each view's Fourier Transform

\begin{tabular}{|l|c|c|c|c|c|c|c|c|}
\hline \multirow{2}{*}{ View } & \multicolumn{4}{|c|}{ Highest testing performance } & \multicolumn{3}{c|}{ Lowest testing performance } \\
\cline { 2 - 10 } & Acc. & Prec. & Sens. & Spec. & Acc. & Prec. & Sens. & Spec. \\
\hline Axial & 85 & 87 & 90 & 75 & 67 & 64 & 47 & 81 \\
\hline Coronal & 80 & 82 & 85 & 74 & 60 & 57 & 57 & 63 \\
\hline Sagittal & 78 & 74 & 96 & 55 & 64 & 62 & 83 & 46 \\
\hline
\end{tabular}

Similar results were seen in the case of GLCOM (combination of contrast, correlation, energy, and homogeneity), where the sagittal view had the lowest performing training. However, the coronal view had much higher training performance than the axial view. Furthermore, the axial view was superior in accuracy, precision, and sensitivity to both views and higher than the lower clinician's values for all parameters, (Table 7.3) 
Table 7.3: Training results for all three views using the GLCOM

\begin{tabular}{|l|c|c|c|c|c|c|c|c|}
\hline \multirow{2}{*}{ View } & \multicolumn{4}{|c|}{ Highest training performance } & \multicolumn{3}{c|}{ Lowest training performance } \\
\cline { 2 - 10 } & Acc. & Prec. & Sens. & Spec. & Acc. & Prec. & Sens. & Spec. \\
\hline Axial & 70 & 72 & 67 & 72 & 64 & 67 & 59 & 71 \\
\hline Coronal & 75 & 76 & 79 & 71 & 69 & 67 & 83 & 52 \\
\hline Sagittal & 63 & 62 & 65 & 63 & 58 & 54 & 72 & 46 \\
\hline
\end{tabular}

In contrast, the axial view had a much higher testing performance outperforming the clinicians and the other two views. The coronal view had comparable accuracy and much greater sensitivity, but its specificity was only higher than the lower limit of the clinicians. On the other hand, the sagittal view was much lower in accuracy, precision, specificity, (Table 7.4).

Table 7.4: Testing results for all three views using the GLCOM

\begin{tabular}{|l|c|c|c|c|c|c|c|c|}
\hline \multirow{2}{*}{ View } & \multicolumn{4}{|c|}{ Highest testing performance } & \multicolumn{3}{c|}{ Lowest testing performance } \\
\cline { 2 - 10 } & Acc. & Prec. & Sens. & Spec. & Acc. & Prec. & Sens. & Spec. \\
\hline Axial & 82 & 86 & 86 & 76 & 76 & 76 & 86 & 67 \\
\hline Coronal & 78 & 78 & 93 & 50 & 69 & 67 & 83 & 52 \\
\hline Sagittal & 69 & 70 & 89 & 35 & 64 & 61 & 67 & 63 \\
\hline
\end{tabular}

Additional to the comparison of views was the comparison of the axial vs the combination of axial plus the other two views. The first combination tested was the correlation, which showed a higher training performance than the single axial view, (Table 7.5). Nevertheless, its performance was lower than the clinicians. Similar results were seen in testing if the combination of views outperformed the single axial view and 
the clinician's performance, (Table 7.6). The values were significantly higher for accuracy, precision, and specificity, and only slightly higher for sensitivity.

Table 7.5: Training results for all three views using the combination of correlation values

\begin{tabular}{|l|c|c|c|c|c|c|c|c|}
\hline \multirow{2}{*}{ View } & \multicolumn{4}{|c|}{ Highest training performance } & \multicolumn{3}{|c|}{ Lowest training performance } \\
\cline { 2 - 9 } & Acc. & Prec. & Sens. & Spec. & Acc. & Prec. & Sens. & Spec. \\
\hline $\begin{array}{l}\text { Axial } \\
\text { correlation }\end{array}$ & 70 & 75 & 59 & 81 & 54 & 61 & 34 & 61 \\
\hline $\begin{array}{l}\text { Combination } \\
\text { (axial+ } \\
\text { coronal+ } \\
\text { sagittal) }\end{array}$ & 72 & 73 & 68 & 76 & 64 & 66 & 76 & 50 \\
\hline
\end{tabular}

Table 7.6: Training results for all three views using the combination of correlation values

\begin{tabular}{|l|c|c|c|c|c|c|c|c|}
\hline \multirow{2}{*}{ View } & \multicolumn{4}{|c|}{ Highest testing performance } & \multicolumn{3}{|c|}{ Lowest testing performance } \\
\cline { 2 - 9 } & Acc. & Prec. & Sens. & Spec. & Acc. & Prec. & Sens. & Spec. \\
\hline $\begin{array}{l}\text { Axial } \\
\text { correlation }\end{array}$ & 78 & 76 & 76 & 79 & 60 & 54 & 74 & 67 \\
\hline $\begin{array}{l}\text { Combination } \\
\text { (axial+ } \\
\text { coronal+ } \\
\text { sagittal) }\end{array}$ & 84 & 85 & 88 & 80 & 79 & 81 & 74 & 83 \\
\hline
\end{tabular}

Another combination tested was the CII combination which included entropy and energy.

The entropy and energy for all views gave a total of 15 values, which were used as inputs. In the case of training, the combination of views outperformed the axial view significantly. It also gave comparable accuracy values and higher specificity values than those of the clinician; the sensitivity value was only greater than the lower range of the clinicians, (Table 7.7). 
Table 7.7: Training results for combination CII (energy and entropy)

\begin{tabular}{|l|c|c|c|c|c|c|c|c|}
\hline \multirow{2}{*}{ View } & \multicolumn{4}{|c|}{ Highest training performance } & \multicolumn{3}{c|}{ Lowest training performance } \\
\cline { 2 - 9 } & Acc. & Prec. & Sens. & Spec. & Acc. & Prec. & Sens. & Spec. \\
\hline $\begin{array}{l}\text { Axial } \\
\text { CII }\end{array}$ & 73 & 68 & 60 & 61 & 57 & 66 & 40 & 75 \\
\hline $\begin{array}{l}\text { Combination } \\
\text { (axial+ } \\
\text { coronal+ } \\
\text { sagittal) }\end{array}$ & 78 & 84 & 73 & 84 & 64 & 64 & 64 & 65 \\
\hline
\end{tabular}

The testing performance was as the correlation combination where the testing performance of the combination was higher than the single axial view. The sensitivity value was only comparable to the lowest clinician's performance. Nevertheless, the accuracy, precision, and specificity values were much higher in comparison to the other views and the clinician's performance, (Table 7.8).

Table 7.8: Testing results for combination CII (energy and entropy)

\begin{tabular}{|l|c|c|c|c|c|c|c|c|}
\hline \multirow{2}{*}{ View } & \multicolumn{4}{|c|}{ Highest testing performance } & \multicolumn{3}{c|}{ Lowest testing performance } \\
\cline { 2 - 9 } & Acc. & Prec. & Sens. & Spec. & Acc. & Prec. & Sens. & Spec. \\
\hline $\begin{array}{l}\text { Axial } \\
\text { Cll }\end{array}$ & 78 & 78 & 88 & 72 & 76 & 76 & 80 & 72 \\
\hline $\begin{array}{l}\text { Combination } \\
\text { (axial+ } \\
\text { coronal+ } \\
\text { sagittal) }\end{array}$ & 80 & 90 & 71 & 91 & 67 & 79 & 48 & 86 \\
\hline
\end{tabular}




\subsection{Statistical results}

The statistical results from the logistical regression model are in concordance with the performance observed during training and testing. In the case of the individual view comparison, all three views resulted in p-values below .05 showing the statistical difference between AD and NC. (Figure 7.1).

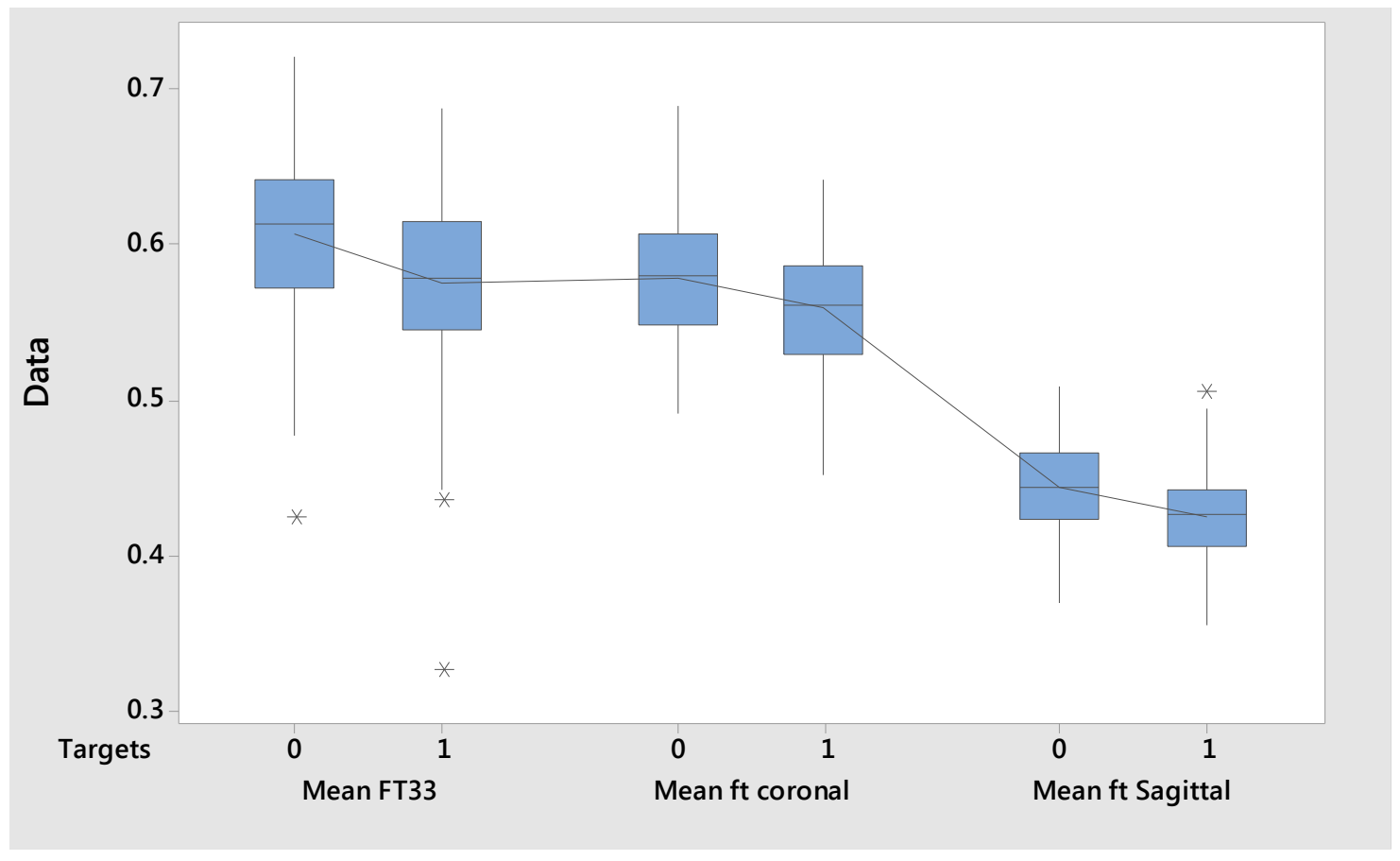

Figure 7.1: Box Plot of NC and AD subject for each view.

The image shows that the means for the groups differ within each of the views. FT33 corresponds to the $3 \times 3$ window from the axial view. Ft Coronal corresponds to the $3 \times 3$ window for the coronal view. Ft Sagittal corresponds to the $3 \times 3$ window for the sagittal view. The 0 is represents normal control groups, the $1 \mathrm{AD}$ groups and the asterisk represents the outliers; there are four outliers total.

The statistical analysis shows that even though each view has a statistical difference among the groups, the views also differ from each other. The axial and coronal 
views are closer to each other, and the sagittal view shows a larger difference in comparison. In the assessment of a single view vs. the combination of views for both correlation and combination CII, a difference was noted among the views. The single view had a larger standard deviation than those in the combination view for both cases of correlation and combination CII, (Appendix G).

\subsection{Summary of results}

The Axial view had a higher performance than the coronal and sagittal views for both training and testing of the Fourier transform and the combination of all texture features (single entity). Furthermore, the lowest performing image view for both cases and both training and testing was the sagittal view. Additionally, the Fourier transform had higher performance values for all views and while compared to the single entity approach. The coronal view performance was greater than the clinicians for all values obtained with the FT, except slightly lower for sensitivity.

In the case that multiple views can increase performance, it was determined that in the event of correlation the training performance was higher for the combination of views. The axial view had much lower values; however, the combination values even though higher, were still below the performance of the clinicians in the case of correlation. During testing, the axial view had only comparable accuracy, higher upperlevel specificity, but lower sensitivity. In contrast, the combination of all views correlation values resulted in much greater testing performance. The testing performance was much higher than the single view, and then the execution of the clinician for all values. Similar results were obtained in the case of combination CII where all the values for energy and entropy were combined for all views resulting in 15 inputs. The 
combination of the views outperformed the single view during both training and testing. In the case of training the axial view's performance was lower than the clinicians; however, the combination view was higher in precision, and specificity, comparable in accuracy, and greater than the lower bound in sensitivity. Moreover, the testing performance outperformed the axial view except for sensitivity which was lower, and only comparable to the clinician's lower bound. On the other hand, the accuracy, precision, and specificity were much higher. Overall, the Fourier transform axial view had a higher performance in both training and testing while compared to all the other techniques, and the clinician's highest performing values. However, the combination of all correlation values for all views was also significantly greater than the single axial view and the performance of the clinicians. 


\section{CHAPTER 8: DISCUSSION}

The primary objective of this study was to investigate if the texture and frequency attributes of MRI images in combination with a feedforward artificial neural network could lead to the classification of $\mathrm{AD}$ in a way that is comparable to the clinician's performance. The studies that reported clinician's performance were limited to, accuracy, sensitivity, and specificity. Thus, the accuracy of the clinicians was determined to be 78 percent based on the number of successes confirmed by autopsy [53]. The sensitivity value for the clinicians had a range of 71 to 87 percent of studies between 2005 and 2010 [54]. Additionally, an average performance of 81 percent was reported from 13 studies published much recently. The same approach gave a specificity range of 44 to 71 percent and an average of 70 percent [54] [55]. There were no studies that report the precision values for the clinicians; however, the precision percent estimated from one of the studies were the exact values for true positive and false positives were given. Thus, the cutoff for precision is at 78 percent similar to accuracy [53]. Moreover, precision was used to distinguish between the highest performing networks. For this work, the best performing ANN structures were those with simultaneous values comparable or greater than those of the clinicians. Thus, the use of artificial neural networks (ANN models) in the combination of a particular frequency and texture attributes can lead to a comparable performance of those of the clinicians.

\subsection{MRI texture and frequency attributes}

One of the main questions being addressed through this study was whether image attributes and frequency domains attributes from MRI images contain valid information for classification of Alzheimer's this work. The comparison of the discrete wavelet 
transform and the Fourier transform at low frequencies showed that the Fourier transform gave higher performance than the DWT. Furthermore, its performance results were comparable and even higher the clinician's performance. The FT low-frequency characteristics to distinguish between $\mathrm{AD}$ and $\mathrm{NC}$ groups were in concordance with other studies. In the case of the Fourier transform and electroencephalogram (EEG), the research showed that there was a higher magnitude in the low-frequency range for $\mathrm{AD}$ individuals that those compared to NC [56]. It is important to note that several studies suggested the use of DWT detail coefficient as attributes rather than the approximation coefficients [57]. Nevertheless, the comparison between the Fourier transform and the DWT in a one to one ratio is only achievable with the low frequencies because the DWT contains both time and frequency components [51]. Another, visible difference between the transforms is the window size and the performance of each transform. For the DWT, the larger window size had higher performing values for training (Table 6.1), and testing which suggests that limiting the window might reduce the information, and therefore reduce performance. On the other hand, the Fourier transform showed almost no difference in the testing performance due to the window size. However, there was a difference during training where the FT achieved 100 percent accuracy, precision, sensitivity, and specificity. Overall, the use of low-frequency domain was much more favorable for the Fourier transform surpassing the clinician's values.

The texture attributes from the images had a larger range of success. The individual texture features when used as inputs resulted in lower performance for both training and testing while compared to the clinicians; however, when used in combination (GLCOM) the contrast, correlation, energy, and homogeneity provided training and 
testing performances comparable and even higher than the clinicians, (Appendix C). The result obtained by the GLCOM gives similar approaches than the case where the GLCOM has been used to increase the sensitivity and specificity of brain tumors; however, one major difference is that these brain studies use a 3D co-occurrence matrix [58]. A similar study using MRI images and GLCOM used a much higher number of attributes a total of 16 but concluded that a larger number of texture features did not improve accuracy [59]. Another comparison was the performance of the GLCOM and the performance of the GLCOM in combination with entropy. The result showed that entropy lowers the performance of the network, which might be a result that entropy cannot be normalized. The value acquired for entropy is a single value; therefore, its normalization will lead to one, which will be insignificant if all entropies are normalized. In the case of the GLCOM, the texture features obtained by the matrix give testing performances higher than the clinicians. A major difference between this study and previous studies is the analysis of the independent features forming the GLCOM. All previous studies had focused on study all features in combination; however, in this approach correlation was identified as a high texture feature that can give comparable performance results to the clinicians. Furthermore, the study of the combination of different features allowed for the understanding of which features can lead to higher performance. Another study determined that the reduction of elements can improve performance; the research suggested that reducing texture features alleviates the effects of high dimensionality, and speeds up retrieval rates [60]. Therefore, a lower number of texture features can have better performances than a combination of multiple features. 
The elements of the GLCOM (contrast, correlation, energy, and homogeneity) had different levels of performance; however, correlation had higher accuracy, sensitivity, and specificity performance in comparison with the clinicians. Additionally, the combination of energy and entropy also had higher sensitivity and specificity performance, and comparable accuracy. Entropy alone performed lower than the clinicians in accuracy, and sensitivity, but higher in specificity. On the other hand, the performance of energy had a higher sensitivity, but lower accuracy and specificity. Thus, the combination of energy and entropy improved performance. Another, study that observed texture attributes in images individually used CT scans, and pulmonary nodules; however, it showed that entropy was higher in affected lungs, and energy was greater in healthy lungs [61]. Such observation might suggest that entropy and energy are opposing measurements. Perhaps, similar results are true in the case of $\mathrm{AD}$, and therefore, their combination is complimenting and leads to higher performance, (Figure 8.1). The graph shows the energy and entropy are different as they contrast with each other; thus, there is a high probability that features opposed to each other, give better performances because they complete each other given a larger range of information. However, a deeper study should be run to confirm these inferences. 


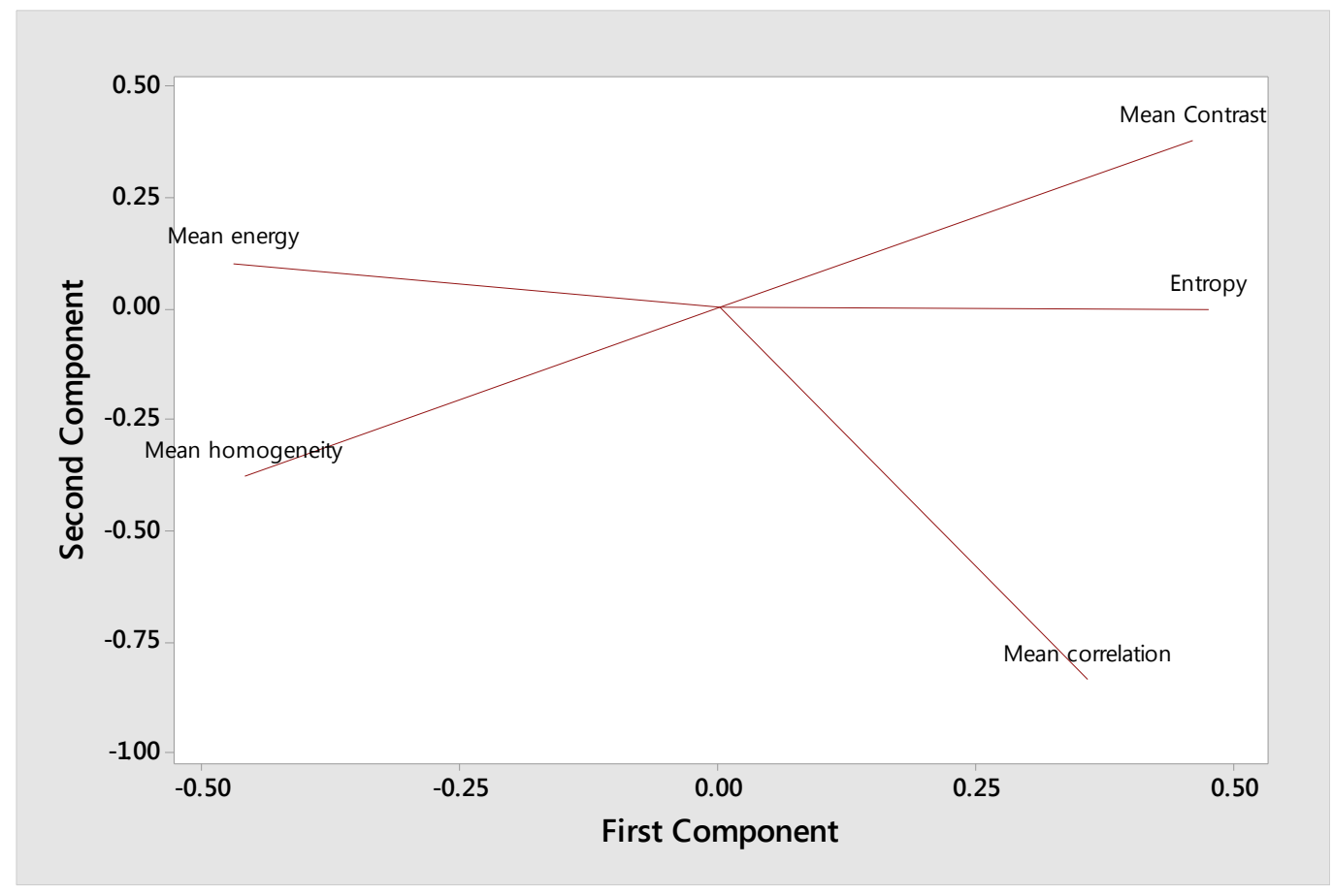

Figure 8.1: Principal Components plot for Texture features.

Lines running parallel to each other are closely related; therefore, there are highly collinear. On the other hand, if the lines are orthogonal, then there is no correlation among the variables. Entropy was the only variable that gave a single value; therefore, there is no mean available. For all other variables, the mean was utilized.

The results obtained from the neural network performance were like those achieved by the logistic regression. The P-values for all means of all texture features showed that there was a statistical difference among the groups; thus, individual texture elements can be used for the differentiation of AD from NC, (Appendix D). 


\subsection{Single view performance vs. combination of views}

The analysis of using one view only for classification or using all three views was another point of investigation in this study. The three views were compared among each other based on the highest performance obtained by the axial view. The highest performing view in the case of FT was the axial view having values greater than those of the clinicians. In comparison with the GLCOM, the axial view showed higher performance than the other two views and was comparable to the clinician's performance. However, this is contrasted to other studies where the favorable image is the coronal view because it provides a much clearer picture than the other two directions [59]. The coronal view did perform higher than the sagittal, and the clinician's performance, but no greater than the axial. One possible reason this study differs from others might be the region selected for analysis. The area size did not affect significantly the performance the FT; however, it influenced the performance of the DWT. The DWT with the larger window had better performance values than the one with the smaller area. Thus, processing the full image, and then selecting a window could change the results. The rationale for choosing a ROI was that there are specific sections of the brain known to be affected by $\mathrm{AD}$, and using those regions only reduces noise. Therefore, the probability of overfeeding the network is reduced, and the likelihood of having useful information increases. The use of any of the views as for classifier was statistically variable, as the p-value for the axial, sagittal, and coronal showed a statistical difference among the groups, (Appendix F).

From the texture features, correlation had the highest performance. The correlation reflects the consistency in the texture of the image, and it has been used to identify 
changes in digital images over time [62]. However, even though the correlation in the axial view outperforms the other views, is only comparable to the performance of the clinicians. On the other hand, the combination of the correlations from all three views significantly increases the performance of the network having superior percentages than the clinicians. Moreover, the statistical analysis showed that there was a difference in the means of the correlation in the axial view, and the combination of correlations,

\section{(Appendix G).}

In the case of combination CII, which is composed of entropy and energy, the results showed that the combination of the features from all three views improved the accuracy, precision, and specificity. However, the specificity was barely comparable to the clinician's lowest performance. The statistical analysis provided similar results where there was a significant difference among the AD and NC. Moreover, there was also a difference between the mean of the single view and the combination of views, (Appendix G). Overall, the combination of the three views provides a higher performance. The use of three views can give the full information of the region being analyzed, and therefore, a more detail information of the area. Similar studies support that use images for analysis had showed that the three views are favorable to reconstruct the full image of the region being observed [63]. The three views provide a three-dimensional information of all the sides of the areas being study; therefore, their combination is desired to increase accuracy. 


\subsection{Network performance}

The use of computer-aided diagnosis systems has been used for the classification of Alzheimer's; however, the combination of texture attributes, frequency attributes of MRI images in conjunction with an ANN model has not yet been studied. Moreover, this study seeks to investigate if the feedforward artificial neural networks (ANN) in combination with the texture and frequency attributes of MRI images can provide a diagnosis comparable to the performance of the clinician. The same values described earlier were used for the comparison: accuracy (78 percent), sensitivity (71-87 percent), and specificity (44-71 percent). The study shows that texture features can be useful for differentiating among groups. The use of correlation measures the linear dependency or the probability of occurrence of particular pixel pairs; thus, previous studies used correlation to observe and identify changes that occurred over time with the use of digital images [3]. Therefore, the greater performance observed in this study using the correlation of the MRI might be detecting the changes that occurred over time due to the progression of $\mathrm{AD}$. The shrinkage or enlargement of voids is a key identifier of AD [62]. Therefore, texture features sensitive to changes in area or mass volume will give higher performances if there are detectable differences between $\mathrm{NC}$ and $\mathrm{AD}$ subjects.

A peculiar finding of this study was the performance of energy and entropy. Both features had low performances when used individually; however, in combination, their performance increased significantly. Energy measures the local uniformity, and entropy the randomness present in the image. They are opposite of each other as shown earlier; however, their combination suggests that they might be complements of each other. 
However, such inference needs to be further evaluated since homogeneity and contrast were also opposite of each other, but their combination did not increase performance. Thus, it might be that is not only enough that the features are different from each other, but the degree of difference and the level of difference in their measurements.

The frequency features obtained from the images show that low frequencies are useful for classification; however, they hinder the performance of the DWT. One limitation to the DWT is the normalization as it is an orthogonal matrix its normalization is much complex than that of the FT. In this study, the normalization of the DWT was performed in the selected window after processing; however, a better method might include an orthogonal normalization or no normalization at all. Thus, in the case of frequency and texture attributes both were used and gave values comparable and higher than those of the clinicians. However, not all texture features and frequencies techniques provide these results.

Additionally, the networks structure and training size influence performance. This study showed that in the case where nine inputs were used the single layer, and fewer neurons performed much higher than with the additional layers and neurons. Regarding larger inputs such as 16 , the single layer with a higher number of neurons performed better (structure 1:35). It is suggested to increase the number of neurons to improve performance [52]. However, there are no studies investigating the relationship between the number of neurons and the volume of inputs. Similarly, there are no studies that looked at the relationship between the training size and its effects on performance. Moreover, forums for professional programmers and artificial neural networks experts have numerous suggestions. There is no clear consensus on how to determine the training 
size other than trial and error. However, this study found that in the cases where training performance was lower the testing performance was much higher. Similarly, in the cases where training was lower testing had much higher performing values; especially, when either training or testing performance values were in the 60 percent. However, the difference in performance was much closer for cases of where the training performance was above 70 percent. A proposed solution to increase both training and testing performance can be increasing the number of samples. A larger number of data samples can give testing and training enough values to perform adequately. There are not studies supporting that such approach would be adequate; however, increasing the sample size will increase performance [52].

In summary, some texture attributes provided comparable performance results to those of the clinicians. The low-frequency transform specifically the FT provided higher performance values than the clinicians. Therefore, both texture and frequency attributes in combinations with a feedforward neural network can perform at the level of the clinician and even higher. 


\section{CHAPTER 9: CONCLUSION AND FUTURE WORK}

\subsection{Conclusion}

Alzheimer's disease is a growing concern due to the population it targets, the rate it affects it and the number of individuals living with the condition. Furthermore, the increasing growing projection rate will cause a substantial economic burden on the individuals, their families, and their countries. Efforts on achieving proper and effective therapies are being studied; however, a significant limitation is the adequate and early diagnosis of the condition. Thus, the use of CAD systems is employed for the analysis of MRI images, and diagnostic classification of AD. However, there are not studies investigating the combination of MRI image attributes, and backpropagation artificial neural networks (ANN). Therefore, this study proposed the use of frequency and texture features from MRI images in combination with the ANN model to classify AD subjects at a comparable rate to the clinicians. This study found that the texture and frequency attributes extracted from the MRI images provided useful information about the picture. Additionally, measures of correlation, energy, and entropy can have higher performance for classification. Moreover, the use of low frequencies from the FT performed far greater than texture attributes and can have better performances regarding specificity, accuracy, sensitivity, and precision, than the clinicians. It was also found that the combination of texture attributes from axial, sagittal, and coronal views improved the performance of the network; however, the axial view outperformed all other views. It was also found that fewer neurons performed better for fewer inputs, and that increasing the number of neurons improve performance for inputs above nine. However, increasing the layers did not improve performance. Furthermore, the training size influenced both 
training and testing performance. The training performance was lower when the testing performance was higher, and vice-versa.

In conclusion, this study showed that texture and frequency attributes in combination with a feedforward artificial neural network could lead to the classification of Alzheimer's disease with a similar and even higher performance of the clinicians. Therefore, the ANN models can help as a second-hand diagnosis to aid the clinicians to increase their level of confidence. However, the limitations of computer aided diagnostic should be noted, and even though high performances are acquired, they are not enough to be a primary diagnostic tool. The clinicians have other skills set that in combination with CAD systems can improve the early diagnosis of AD.

\subsection{Future work}

Time and resources limited this study; therefore, there are still investigative inquiries that can be further addressed. Additional texture features and combinations can be used to determine the relationship of combinations of elements and their relationship regarding their different measurements, such in the case of entropy and energy. Another inquiry would be the normalization of the DWT by a means that not lead to only zeroes. Furthermore, the combination of DWT and FT can be study to determine if performance is increased by combing both frequencies. Likewise, to study if the combination of the transforms from all three views also increase performance as in the case of texture features. A further study is necessary to compare the use of different programming languages to determine if the performance is limited by the capabilities of the software and not the data. Another study can include to increase the sample size, and to add a group such as Parkinson patients to study if the classification performance is still 
comparable to the clinicians and if it is sensitive to overlapping regions. A significant improvement of this study will be the use of MRI images of individuals who are known to have $\mathrm{AD}$ or have a higher probability of $\mathrm{AD}$, but whose symptoms are non-present. $\mathrm{A}$ superior performing ANN model with such population could suggest that the ANN models could improve the early diagnosis of the disease, and therefore increase the probability of successful treatments. 


\section{REFERENCES}

[1] WHO, "dementia fact sheet," 2016. [Online]. [Accessed 231 2017].

[2] NIA National Institute on Aging \& NIH National Institute of Helath, "Alzheimer's Disease Progress Report, Intensifying the Research Effort," U.S. Department fo Health and Human Services, Maryland, 2011-2012.

[3] Alzheimer's Association, "2016 Alzheimer's disease facts and figures," Alzheimer's \& Dementia, vol. 12, no. 4, pp. 459-509, 2016.

[4] Alzheimer's Association, "10 Early Signs and Symptoms of Alzheimer's," 2017. [Online]. Available: www.alz.org. [Accessed 15 January 2017].

[5] van der Laan, Anna Laura, "Grey Matters: Emergent Biomarkers and Good Alzheimer's Diagnostics.," In Emerging Technologies for Diagnosing Alzheimer's Disease, no. Palgrave Macmillan UK, pp. 103-121, 2016.

[6] Doi, Kunio, "Computer-aided diagnosis in medical imaging: historical review, current status and future potential," Computerized medical imaging and graphics, vol. 31, no. 4, pp. 198-211, 2007.

[7] World Health Organization, "Early cancer diagnosis, better trained medics can save lives, money," 2017.

[8] Alzheimer's Disease International, "The Global Voice on Dementation Statistics," Alzheimer's Disease International (ADI), London.

[9] " 2016 Alzheimer's disease facts and figures. Alzheimer's \& Dementia," vol. 12, no. 4, pp. 459-509, 2016.

[10] Lin, Ai-Ling, Angela R. Laird, Peter T. Fox, and Jia-Hong Gao, "Multimodal MRI neuroimaging biomarkers for cognitive normal adults, amnestic mild cognitive impairment, and Alzheimer's disease," Neurology research international, vol. 2012, no. $907409,2012$.

[11] Reiman, Eric M., Kewei Chen, Gene E. Alexander, Richard J. Caselli, Daniel Bandy, David Osborne, Ann M. Saunders, and John Hardy, "Functional brain abnormalities in young adults at genetic risk for late-onset Alzheimer's dementia," Proceedings of the National Academy of Sciences, vol. 101, no. 1, pp. 284-289, 
2004.

[12] Gaugler, Joseph E., Haya Ascher-Svanum, David L. Roth, Tolulope Fafowora, Andrew Siderowf, and Thomas G. Beach, "Characteristics of patients misdiagnosed with Alzheimer's disease and their medication use: an analysis of the NACC-UDS database," BMC geriatrics, vol. 13, no. 1, p. 137, 2013.

[13] Guest, Paul C, "Biomarkers and Mental Illness: It's Not All in the Mind," Springe, 2017.

[14] Liu, Siqi, Sidong Liu, Weidong Cai, Sonia Pujol, Ron Kikinis, and Dagan Feng, "Early diagnosis of Alzheimer's disease with deep learning. In Biomedical Imaging," Biomedical Imaging (ISBI), vol. IEEE 11th International Symposium, pp. 10151018, 2014.

[15] aël Chetelat, G., and Jean-Claude Baron, "Early diagnosis of Alzheimer's disease: contribution of structural neuroimaging," Neuroimage, vol. 18, no. 2, pp. 525-541, 2003.

[16] Hudson, Brian J. Balin \& Alan P., "Etiology and Pathogenesis of Late-Onset Alzheimer's Disease," Current allergy and asthma reports, vol. 14, no. 3, p. 10, 2014.

[17] Damian Gallegher, Robert F. Coen, and Brian A. Lawlor, "Alzheimer's Disease," in Neurodegenerative Disorders: A Clinical Guide, Switzerland, Springer International, 2016, pp. 57-74.

[18] Ates, M. P., Karaman, Y., Guntekin, S., \& Ergun, M. A., "Analysis of genetics and risk factors of Alzheimer's disease," Neuroscience, vol. 325, pp. 124-131, 2016.

[19] T. K. H. X. G. B. Chia-Chen Liu, "Apolipoprotein E and Alzheimer disease: risk, mechanisms, and therapy," Nature reviews Neurology, vol. 9, no. 2, pp. 106-118, 2013.

[20] Sunderland, Trey, et al., "'Decreased $\beta$-amyloid1-42 and increased tau levels in cerebrospinal fluid of patients with Alzheimer disease."," Jama, vol. 289.16, pp. 2094-2103, 2003.

[21] Gonzalez, Manuel Menendez, Atlas of Biomarkers for Alzheimer's Disease, Switzerland: Springer International Publishing, 2014. 
[22] DeCarlo, Correne A., Stuart WS MacDonald, David Vergote, Jack Jhamandas, David Westaway, and Roger A. Dixon., ""Vascular health and genetic risk affect mild cognitive impairment status and 4-year stability: Evidence from the Victoria Longitudinal Study."," The Journals of Gerontology Series B: Psychological Sciences and Social Sciences, vol. 71, no. 6, pp. 1004-1014., 2016.

[23] Renee Prajer, RDH, MS and Mark Kacerik, RDH, MS, "Early Vs Late Onset," The Journal of Professsional Excellence, vol. 4, no. 9, 2006.

[24] Dubois, B., Feldman, H.H., Jacova, C., DeKosky, S.T., Barberger-Gateau, P., Cummings, J., Delacourte, A., Galasko, D., Gauthier, S., Jicha, G. and Meguro, K., "Research criteria for the diagnosis of Alzheimer's disease: revising the NINCDSADRDA criteria," The Lancet Neurology, vol. 6, no. 8, pp. 734-746, 2007.

[25] McKhann, Guy, David Drachman, Marshall Folstein, Robert Katzman, Donald Price, and Emanuel M. Stadlan, "Clinical diagnosis of Alzheimer's disease Report of the NINCDS-ADRDA Work Group* under the auspices of Department of Health and Human Services Task Force on Alzheimer's Disease," Neurology, vol. 34, no. 7, pp. 939-939, 1984.

[26] Snyder, Heather M., and Maria C. Carrillo, "Perspective from the Alzheimer's Association: Neuroimaging Professional Interest Area of ISTAART continues impact on the field," Alzheimer's \& Dementia: Diagnosis, Assessment \& Disease Monitoring, vol. 5, no. 1, 2016.

[27] California Department of Health Care Services Community Based Adult Services, "Guide to Determine Alzheimer Disease or Dementia Stages," State of California, 2011.

[28] Biasutti, Maria, Natacha Dufour, Clotilde Ferroud, William Dab, and Laura Temime, "Cost-effectiveness of magnetic resonance imaging with a new contrast agent for the early diagnosis of Alzheimer's disease," PloS one, vol. 7, no. 4, 2012.

[29] Nishikawa, Robert M, "Current status and future directions of computer-aided diagnosis in mammography.," Computerized Medical Imaging and Graphics, vol. 31, no. 4, pp. 224-235, 2007.

[30] Firmino, Macedo, Giovani Angelo, Higor Morais, Marcel R. Dantas, and Ricardo Valentim, "Computer-aided detection (CADe) and diagnosis (CADx) system for lung cancer with likelihood of malignancy," Biomedical engineering online, vol. 15, no. 1, p. 17, 2016. 
[31] Yang, Hsuan-Chia, Richard Lu, Chieh-Chen Wu, and Yu-Chuan Jack Li, "Reliable and stable computer-aided diagnosis systems for images," Reliable and stable computer-aided diagnosis systems for images, vol. 128, pp. A1-A2,158, 2016.

[32] Meeuwis, Carla, Stephanie M. van de Ven, Gerard Stapper, Arancha M. Fernandez Gallardo, Maurice AAJ van den Bosch, P. Th M. Willem, and Wouter B. Veldhuis, "Computer-aided detection (CAD) for breast MRI: evaluation of efficacy at 3.0 T," European radiology, vol. 20, no. 3, pp. 522-528, 2010.

[33] Hsieh, Kevin Li-Chun, Ruei-Je Tsai, Yu-Chuan Teng, and Chung-Ming Lo, "Effect of a computer-aided diagnosis system on radiologists' performance in grading gliomas with MR," PloS one, vol. 12, no. 2, 2017.

[34] Ramaniharan, Anandh Kilpattu, Sujatha Chinnaswamy Manoharan, and Ramakrishnan Swaminathan, "Laplace Beltrami eigen value based classification of normal and Alzheimer MR images using parametric and non-parametric classifiers," Expert Systems with Applications, vol. 15, no. 59, pp. 208-216., 2016.

[35] Iman Beheshtia, Hasan Demirelb, Farnaz Farokhianc, Chunlan Yangc, Hiroshi Matsudaa, "Structural MRI-based detection of Alzheimer's disease using feature ranking and classification error," Computer Methods and Programs in Biomedicine, vol. 137, p. 177-193, 2016.

[36] Rajeesh, J., R. S. Moni, and S. S. Kumar., "Performance analysis of wave atom transform in texture classification," Signal, vol. 8, no. 5, pp. 923-30, 2014.

[37] Chaves, Rosa, Javier Ramírez, J. M. Gorriz, and Alzheimer's Disease Neuroimaging Initiative., "Integrating discretization and association rule-based classification for Alzheimer's disease diagnosis.," "Expert Systems with Applications, vol. 40, no. 5, pp. 1571-1578, 2013.

[38] Patil, M. M., and A. R. Yardi, "Classification of 3D Magnetic Resonance Images of Brain using Discrete Wavelet Transform," International Journal of Computer Applications, vol. 31, no. 7, pp. 23-27, 2011.

[39] Gonzalez, Rafael C. , Woods Richard E., Digital Image Processing (3rd Edition), New Jersey: Hall, 2007.

[40] Cai, Suping, Yuanyuan Jiang, Yubo Wang, Xiaoming Wu, Junchan Ren, Min Seob Lee, Sunghoon Lee, and Liyu Huang., "Modulation on brain gray matter activity and white matter integrity by APOE $\varepsilon 4$ risk gene in cognitively intact elderly: A 
multimodal neuroimaging study," Behavioural brain research, vol. 30, no. 322, pp. 100-9, 2017.

[41] Jack, Clifford R., Matt A. Bernstein, Nick C. Fox, Paul Thompson, Gene Alexander, Danielle Harvey, Bret Borowski, "The Alzheimer's disease neuroimaging initiative (ADNI): MRI methods," 1 April 2008. [Online]. [Accessed 2016].

[42] The MathWorks, Inc., "Image processing Toolbox: User's Guide," The MathWorks, Inc., Natick, MA, 2017.

[43] Johnson, Keith A., Nick C. Fox, Reisa A. Sperling, and William E. Klunk, "Brain imaging in Alzheimer disease," Cold Spring Harbor perspectives in medicine, vol. 2, no. 4:a006213, 2012.

[44] Mu, Yangling, and Fred H. Gage, "Adult hippocampal neurogenesis and its role in Alzheimer's disease," Molecular neurodegeneration, vol. 6, no. 1, p. 85, 2011.

[45] L. W. de Jong K. van der Hiele I. M. Veer J. J. Houwing R. G. J. Westendorp E. L. E. M. Bollen P. W. de Bruin H. A. M. Middelkoop M. A. van Buchem J. van der Grond, "Strongly reduced volumes of putamen and thalamus in Alzheimer's disease: an MRI study," Brain, vol. 131, no. 12, pp. 3277-85, 2008.

[46] Apostolova, Liana G., Amity E. Green, Sona Babakchanian, Kristy S. Hwang, YiYu Chou, Arthur W. Toga, and Paul M. Thompson, "Hippocampal atrophy and ventricular enlargement in normal aging, mild cognitive impairment and Alzheimer's disease," Alzheimer disease and associated disorders, vol. 26, no. 1, 2012.

[47] S. Haykin, Neural Networks and Learning Machines, New Jersey: Pearson Prentice Hall, 2009.

[48] Dieterle, Frank;, "Dr. Frank Dieterle," 148 2006. [Online]. [Accessed 2017].

[49] Farhan, Saima, Muhammad Abuzar Fahiem, and Huma Tauseef, "Farhan, S., Fahiem, M. A., \& Tauseef, H. (2014). An ensemble-of-classifiers based approach for early diagnosis of Alzheimer's disease: Classification using structural features of brain images," Computational and mathematical methods in medicine, 2014.

[50] Aruna, S., S. P. Rajagopalan, Dr., and L. V. Nandakishore., "Knowledge based analysis of various statistical tools in detecting breast cancer," Computer Science \& Information Technology, vol. 2, pp. 37-45, 2011. 
[51] Klapetek, Petr. Necas, David. Anderson, Christophier., "Wavelet Transform," in Data Processing and Analysis, Czech, Czech Metrology Institute, 2016.

[52] MathWorks, "Statistics and Machine Learning toolbox: Performance Curves," The MathWorks, Inc., 2017.

[53] Winnie Qian, BSc, David Munoz, MD, "Misdiagnosis of Alzheimer's disease: inconsistencies between clinical diagnosis and neuropathological confirmation," in Alzheimer's Association International Conference, Ontario, Canada, 2016.

[54] Beach, T.G., Monsell, S.E., Phillips, L.E. and Kukull, W, "Accuracy of the clinical diagnosis of Alzheimer disease at National Institute on Aging Alzheimer Disease Centers," Journal of neuropathology and experimental neurology, vol. 71, no. 4, pp. 266-273, 2012.

[55] Klöppel, S., Stonnington, C.M., Chu, C., Draganski, B., Scahill, R.I., Rohrer, J.D., Fox, N.C., Jack Jr, C.R., Ashburner, J. and Frackowiak, R.S., "Automatic classification of MR scans in Alzheimer's disease," Brain, vol. 131, no. 3, pp. 681689, 2008.

[56] Dierks, T., Ihl, R., Frölich, L. and Maurer, K.,, "Dementia of the Alzheimer type: effects on the spontaneous EEG described by dipole sources.," Psychiatry Research: Neuroimaging, vol. 50, no. 3, pp. 151-162, 1993.

[57] Zhang, Y., Dong, Z., Phillips, P., Wang, S., Ji, G., Yang, J. and Yuan, T.F.,, "Detection of subjects and brain regions related to Alzheimer's disease using 3D MRI scans based on eigenbrain and machine learning," Frontiers in Computational Neuroscience, vol. 9, 2015.

[58] Mahmoud-Ghoneim, D., Toussaint, G., Constans, J.M. and Jacques, D., "Three dimensional texture analysis in MRI: a preliminary evaluation in gliomas," Magnetic resonance imaging, vol. 21, no. 9, pp. 983-987., 2003.

[59] Zhang, J., Yu, C., Jiang, G., Liu, W. and Tong, L., "3D texture analysis on MRI images of Alzheimer's disease," Brain imaging and behavior, vol. 6, no. 1, pp. 6169, 2012.

[60] Antonio Catalan, J., Jin, J.S. and Gedeon, T., "Reducing the dimensions of texture features for image retrieval using multi-layer neural networks," Pattern Analysis \& Applications, vol. 2, no. 2, pp. 196-203, 1999. 
[61] Zhao, Q., Shi, C.Z. and Luo, L.P., "Role of the texture features of images in the diagnosis of solitary pulmonary nodules in different sizes," Chinese Journal of Cancer Research, vol. 26, no. 4, 2014.

[62] McCormick, N. and Lord, J., "Digital image correlation," Materials today, vol. 13, no. 12 , pp. 52-54, 2010.

[63] Lefevre, N., Naouri, J.F., Herman, S., Gerometta, A., Klouche, S. and Bohu, Y., "A current review of the meniscus imaging: proposition of a useful tool for its radiologic analysis," Radiology research and practice, 2016.

[64] Harper, Lorna, Giorgio G. Fumagalli, Frederik Barkhof, Philip Scheltens, John T. O'Brien, Femke Bouwman, Emma J. Burton et al, "MRI visual rating scales in the diagnosis of dementia: evaluation in 184 post-mortem confirmed cases," Brain, 2016.

[65] Verhagen, Martijn V., Gerard L. Guit, Gerrit Jan Hafkamp, and Kees Kalisvaart, "The impact of MRI combined with visual rating scales on the clinical diagnosis of dementia: a prospective study," European radiology, vol. 26, no. 6, pp. 1716-1722, 2016. 


\section{APPENDICES}

\section{APPENDIX A}

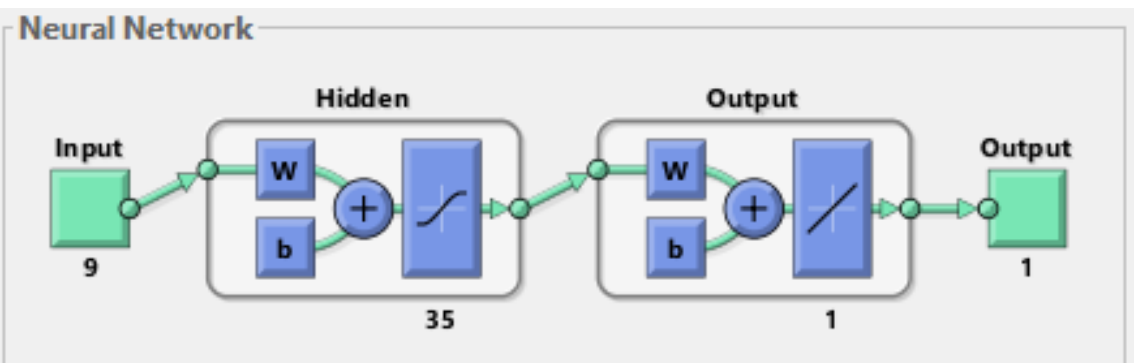

Figure A. 1: Structure 1:35 with 9 inputs and 1 output
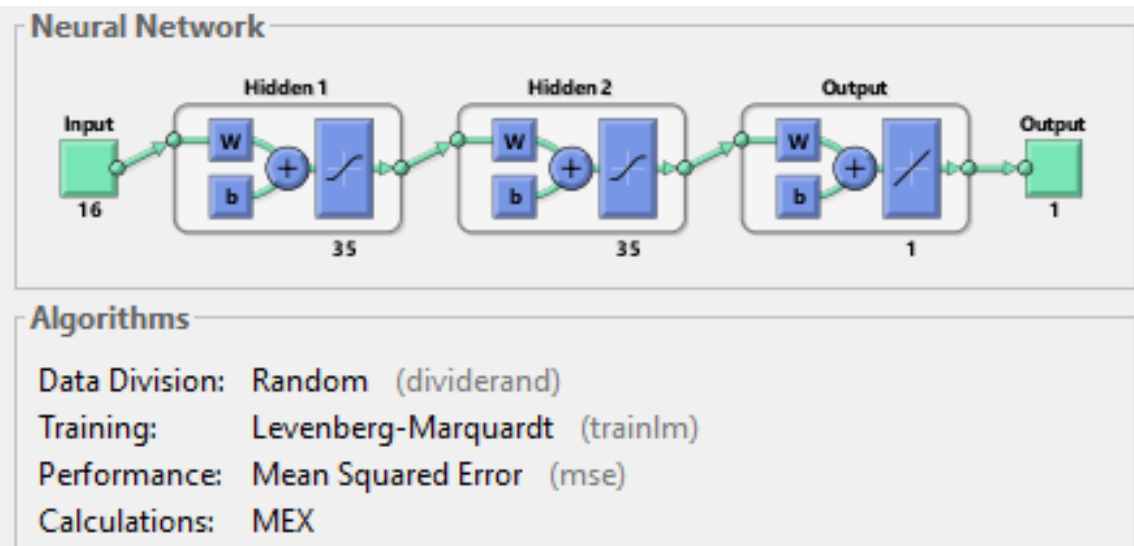

\section{Figure A. 2: Structure 2:35 with 16 inputs and 1 output.}

This structure has two hidden layers and each layer contains 35 neurons. There is one output only. 
APPENDIX A (CONT.)

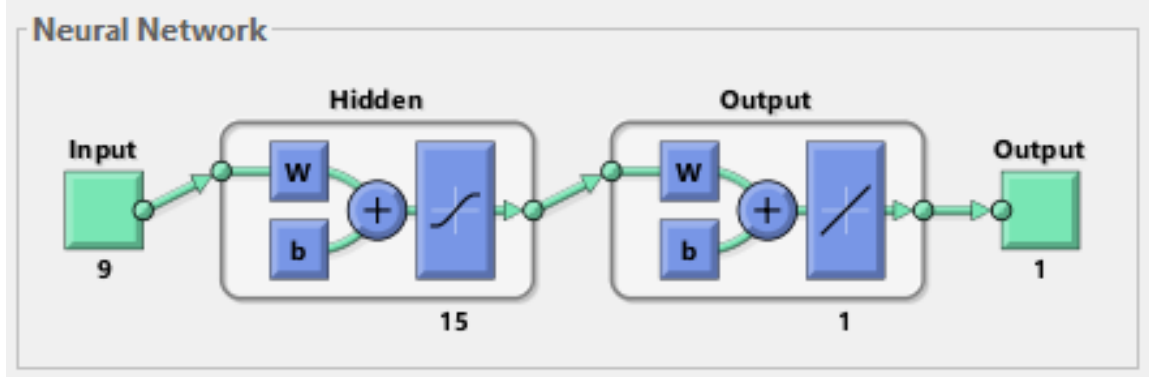

Figure A. 3: Structure 1:15 with 9 inputs and 1 output.

This structure has one hidden layer and 15 neurons with in the hidden layers. Here are 9 inputs and 1 output; however, the number of inputs can be increase or decrease. Though, the expected output will always be one. 


\section{APPENDIX B}
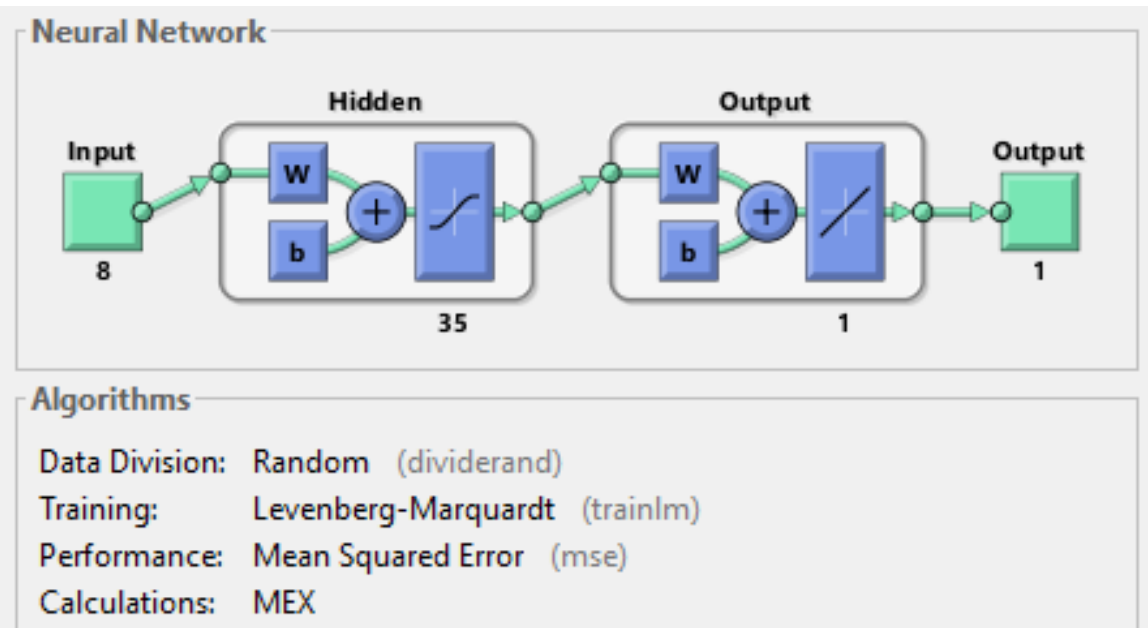

Figure B. 1: Structure 1:35 with 8 inputs and 1 output (combination of any two

features except entropy). Entropy only adds one input; therefore, the number of inputs will change to 5 . 


\section{APPENDIX C}

Table C. 1: Group A definition of combinations

\begin{tabular}{|l|l|}
\hline $\begin{array}{l}\text { Combination } \\
\text { Group and } \\
\text { Number }\end{array}$ & Features combined \\
\hline Al & $\begin{array}{l}\text { Contrast and } \\
\text { Correlation }\end{array}$ \\
\hline All & Contrast and Energy \\
\hline AllI & $\begin{array}{l}\text { Contrast and } \\
\text { Homogeneity }\end{array}$ \\
\hline AIV & Contrast and Entropy \\
\hline
\end{tabular}

Table C. 2: Group B definition of combinations

\begin{tabular}{|l|l|}
\hline $\begin{array}{l}\text { Combination } \\
\text { Group and } \\
\text { Number }\end{array}$ & Features combined \\
\hline $\mathrm{BI}$ & \begin{tabular}{l} 
Correlation and Energy \\
\hline BII
\end{tabular} \\
\hline BIII & $\begin{array}{l}\text { Correlation and } \\
\text { Homogeneity }\end{array}$ \\
\hline
\end{tabular}

Table C. 3: Group C definition of combinations

\begin{tabular}{|l|l|}
\hline $\begin{array}{l}\text { Combination } \\
\text { Group and } \\
\text { Number }\end{array}$ & Features combined \\
\hline $\mathrm{Cl}$ & $\begin{array}{l}\text { Energy and } \\
\text { Homogeneity }\end{array}$ \\
\hline $\mathrm{Cll}$ & Energy and Entropy \\
\hline
\end{tabular}


Table C. 4: Group D definition of combinations

\begin{tabular}{|l|l|}
\hline $\begin{array}{l}\text { Combination } \\
\text { Group and } \\
\text { Number }\end{array}$ & Features combined \\
\hline DI & $\begin{array}{l}\text { Homogeneity and } \\
\text { Entropy }\end{array}$ \\
\hline
\end{tabular}




\section{APPENDIX D}

$\begin{array}{ll}\text { Targets } & 1 \\ & 0 \\ & \text { Total } \\ & \\ \text { Deviance Table } & \\ \text { Source } \\ \text { Regression } \\ \text { Mean Contrast } \\ \text { Mean correlation } \\ \text { Mean energy } \\ \text { Entro } \\ \text { Gender } \\ \text { Error } \\ \text { Total } \\ \text { | }\end{array}$

150
150

300
(Event)

DF

29.649

7.669

7.429

0.481

14.051

0.019

386.239

415.888

$\begin{array}{rr}\text { Contribution } & \text { Adj Dev } \\ 7.13 \% & 29.649 \\ 1.84 \% & 5.042 \\ 1.79 \% & 7.085 \\ 0.12 \% & 5.071 \\ 3.38 \% & 13.989 \\ 0.00 \% & 0.019 \\ 92.87 \% & 386.239 \\ 100.008 & \end{array}$

$\begin{array}{rrr}\text { Adj Mean } & \text { Chi-Square } & \text { P-Value } \\ 5.9298 & 29.65 & 0.000 \\ 5.0421 & 5.04 & 0.025 \\ 7.0850 & 7.09 & 0.008 \\ 5.0709 & 5.07 & 0.024 \\ 13.9887 & 13.99 & 0.000 \\ 0.0186 & 0.02 & 0.892\end{array}$




\section{APPENDIX E}

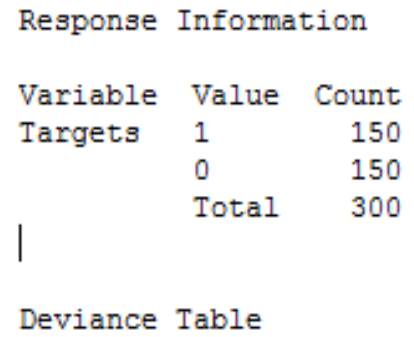

$\begin{array}{rr}\text { Chi-Square } & \text { P-Value } \\ 54.14 & 0.000 \\ 41.54 & 0.000 \\ 3.46 & 0.063 \\ 2.23 & 0.135 \\ 0.05 & 0.821\end{array}$

$\begin{array}{lrrrrrrr}\text { Term } & \text { Coef } & \text { SE Coef } & & 95 \text { \% CI } & \text { Z-Value } & \text { P-Value } & \text { VIF } \\ \text { Constant } & -8.69 & 3.13 & (-14.83, & -2.55) & -2.78 & 0.006 & \\ \text { mean ft44 } & -18.00 & 3.15 & (-24.17, & -11.82) & -5.71 & 0.000 & 1.59\end{array}$




\section{APPENDIX F}

Nested ANOVA: Mean ft coronal versus Targets, Gender

$\begin{array}{lrrrrr}\text { Analysis of Variance for Mean ft coronal } \\ & & & & & \\ \text { Source } & \text { DF } & \text { SS } & \text { MS } & \text { F } & \text { P } \\ \text { Targets } & 1 & 0.0295 & 0.0295 & 27.526 & 0.034 \\ \text { Gender } & 2 & 0.0021 & 0.0011 & 0.627 & 0.535 \\ \text { Error } & 296 & 0.5062 & 0.0017 & & \\ \text { Total } & 299 & 0.5379 & & & \end{array}$

Nested ANOVA: Mean ft Sagittal versus Targets, Gender

$\begin{array}{lrrrrr}\text { Analysis of Variance for Mean ft Sagittal } & \\ & & & & \text { F } & \text { P } \\ \text { Source } & \text { DF } & \text { SS } & \text { MS } & \text { F } & 0.007 \\ \text { Targets } & 1 & 0.0240 & 0.0240 & 151.291 & 0.04 \\ \text { Gender } & 2 & 0.0003 & 0.0002 & 0.193 & 0.824 \\ \text { Error } & 296 & 0.2432 & 0.0008 & & \\ \text { Total } & 299 & 0.2676 & & & \end{array}$




\section{APPENDIX G}

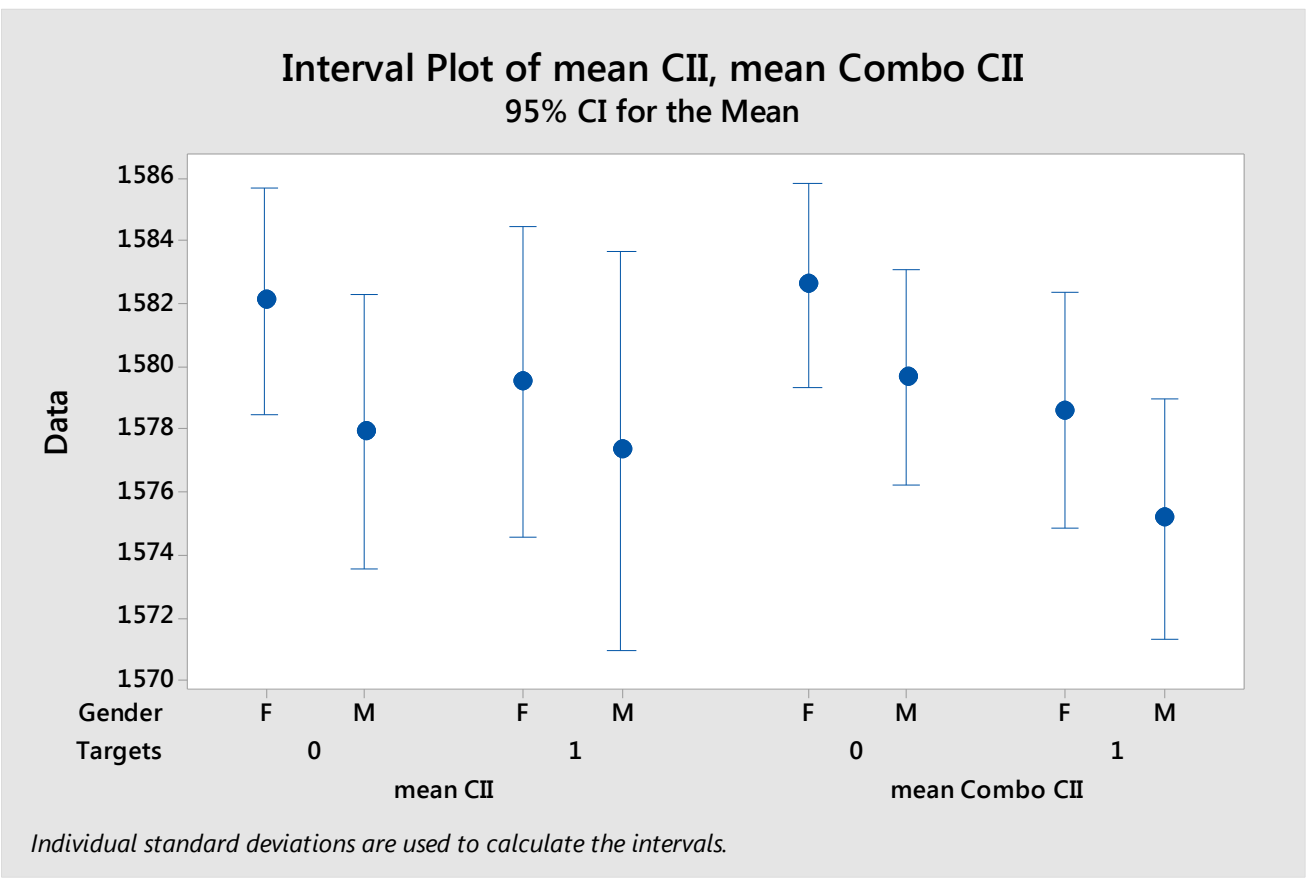

Figure G. 1: Comparison plot of single view and combination view of CII.

The figure shows the difference between the means of CII (Energy + Entropy) by group and gender. 0 is for normal controls (NC), and 1 is for $\mathrm{AD}$ patients. The mean for the single view (Left side) shows that there is a difference among the groups $\mathrm{NC}$ and $\mathrm{AD}$, and among the Males and females. The combination of multi-view showed similar results; however, the differences between the NC and AD groups are much pronounced. In both cases, the mean among males from $\mathrm{NC}$ and females for $\mathrm{AD}$ are closer together. 


\section{APPENDIX G (CONT.)}

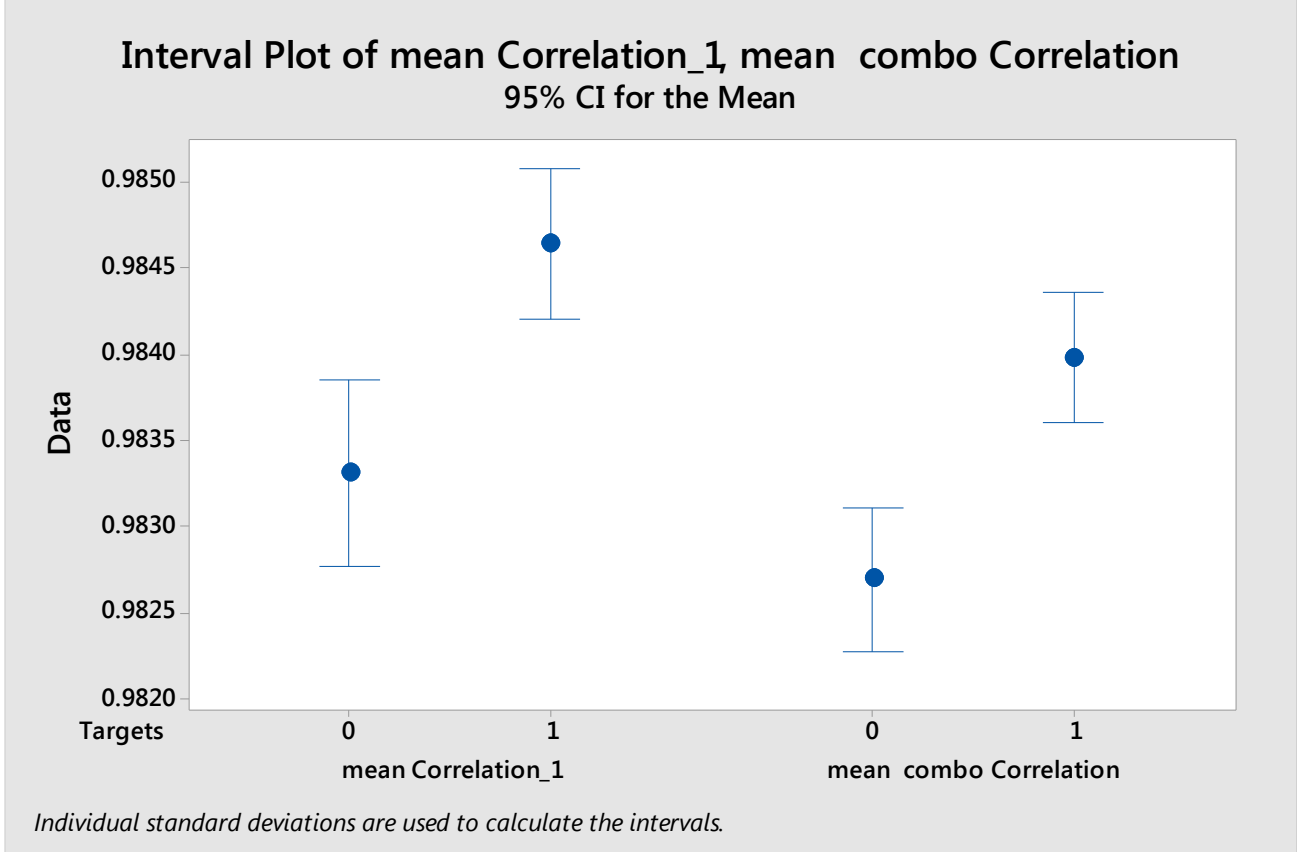

Figure G. 2: Correlation Comparison Plot single view vs. combination view.

The figure represents the means of a single view (left side), and by groups, 0 represents normal controls (NC), and 1 represents $\mathrm{AD}$ group. The right side shows the means for the combination of multi-views (axial, sagittal, and coronal views). The images depict a clear difference among the groups' means for both cases where the NC has a lower mean in both instances. In the comparison between single and multi-view, there is also a difference between the average values; the combination view resulted in lower means for both $\mathrm{AD}$ and $\mathrm{NC}$ groups. 